\title{
Economic Nonlinear Model Predictive Control
}

Timm Faulwasser

Karlsruhe Institute of Technology (KIT)

timm.faulwasser@kit.edu

Lars Grüne

University of Bayreuth

lars.gruene@uni-bayreuth.de

Matthias A. Müller

University of Stuttgart

matthias.mueller@ist.uni-stuttgart.de 


\begin{abstract}
In recent years, Economic Model Predictive Control (EMPC) has received considerable attention of many research groups. The present tutorial survey summarizes state-of-the-art approaches in EMPC. In this context EMPC is to be understood as receding-horizon optimal control with a stage cost that does not simply penalize the distance to a desired equilibrium but encodes more sophisticated economic objectives.

This survey provides a comprehensive overview of EMPC stability results: with and without terminal constraints, with and without dissipativtiy assumptions, with averaged constraints, formulations with multiple objectives and generalized terminal constraints as well as Lyapunov-based approaches. Moreover, we compare different performance criteria for some of the considered approaches and comment on the connections to recent research on dissipativity of optimal control problems. We consider a discrete-time setting and point towards continuous-time variants. We illustrate the different EMPC schemes with several examples.
\end{abstract}




\section{Contents}

\begin{tabular}{lll}
\hline & Introduction & 5
\end{tabular}

1.1 Outline . . . . . . . . . . . . . . . . . . . . . . 6

1.2 Notation . . . . . . . . . . . . . . . . . . . . . 6

$\begin{array}{lll}2 & \text { Revisiting Stabilizing NMPC } & 9\end{array}$

2.1 Main Idea of NMPC $\ldots \ldots \ldots \ldots$

2.2 Stabilizing NMPC with Terminal Constraints . . . . . . . . . . . . . . 11

2.3 Stabilizing NMPC without Terminal Constraints . . . . . . . . . . . . . . 14

3 Economic MPC with Terminal Constraints 17

3.1 Dissipativity and Optimal Operation at Steady State . . . . . . . . . . . 18

3.2 Closed-loop Stability . . . . . . . . . . . . . . . . . . . . . 21

3.3 Example - Chemical Reactor with Dissipativity . . . . . . . . . . . . . . . . 23

$3.4 \quad$ Example - Chemical Reactor without Dissipativity . . . . . . . . . . . . 26

4 EMPC without Terminal Constraints and Penalties 31

4.1 The Turnpike Property . . . . . . . . . . . . . . . . . . . . . . . . . . 31

4.2 Recursive Feasibility . . . . . . . . . . . . . . . . . . . . . . . . 34

4.3 Practical Stability $\ldots \ldots \ldots$. . . . . . . . . . . . . . . . . . . . 36

$4.4 \quad$ Example - Chemical Reactor with Dissipativity . . . . . . . . . . . . . . 42

5 Performance Bounds $\quad 45$

5.1 Averaged Performance . . . . . . . . . . . . . . . . . . . . 46

5.2 Non-averaged Performance . . . . . . . . . . . . . . . . . . . . . 47

5.3 Transient Performance . . . . . . . . . . . . . . . . . . . . . . . . . . . . 48

5.4 Example - Chemical Reactor with Dissipativity . . . . . . . . . . . . . . 49

6 EMPC with Averaged Constraints

6.1 Asymptotic Average Constraints . . . . . . . . . . . . . . . . . . . . 52

6.2 Simple Example . . . . . . . . . . . . . . . . . . . . . . . . . 57

6.3 Transient Average Constraints . . . . . . . . . . . . . . . . . . . . . 58

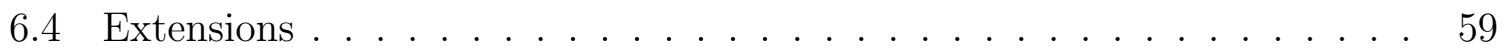

7 EMPC with Generalized Terminal Constraints $\quad 61$

7.1 Problem Formulation and Performance Analysis . . . . . . . . . . . . . . 62

7.2 Self-tuning Terminal Weight $\ldots \ldots \ldots \ldots$. . . . . . . . . . 65 
7.3 Discussion and Extensions f . . . . . . . . . . . . . . . . . . . 67

8 Lyapunov-based Approach $\quad 69$

8.1 Basics of the Scheme . . . . . . . . . . . . . . . . . . . . . . . . 69

8.2 Closed-loop Properties . . . . . . . . . . . . . . . . . . . . 71

\begin{tabular}{lll}
\hline & Multi-objective Approach & 75
\end{tabular}

9.1 Derivation of the Scheme . . . . . . . . . . . . . . . . . . . . . . . 75

9.2 Closed-loop Properties . . . . . . . . . . . . . . . . . . . . . 77

9.3 Example - Chemical Reactor without Dissipativity . . . . . . . . . . . . 78

10 Conclusions and Outlook $\quad 81$

10.1 Discussion . . . . . . . . . . . . . . . . . . . . . . . . 81

10.2 Further Results and Open Problems. . . . . . . . . . . . . . . . . 83 


\section{Author Contributions}

All authors have contributed equally to the preparation of this survey.

\section{Funding Acknowledgments}

Timm Faulwasser acknowledges support

- of his research by the Helmholtz Association under the Joint Initiative "Energy System 2050 - A Contribution of the Research Field Energy",

- from the Baden-Württemberg Stiftung under the Elite Programme for Postdocs, and

- from the Deutsche Forschungsgemeinschaft, Grants WO 2056/1 and WO 2056/4-1.

Lars Grüne acknowledges support

- from the Deutsche Forschungsgemeinschaft, Grants GR 1569/13-1, GR 1569/15-1 and GR 1569/16-1.

Matthias A. Müller acknowledges support

- from the Deutsche Forschungsgemeinschaft, Grants MU 3929/1-1, WO 2056/1, and WO 2056/4-1, and

- from the Baden-Württemberg Stiftung under the Elite Programme for Postdocs. 



\section{Introduction}

The principle idea of Model Predictive Control (MPC) can be dated back to the 1960s, when Propoi, 1963 as well as Lee and Markus, 1967 suggested receding-horizon solutions of Optimal Control Problems (OCP). While MPC saw its first applications in petro-chemical industries in the 1970s, by now a mature body of knowledge encompasses stability and robustness of linear and nonlinear MPC 1 1 strategies and tools for efficient numerical implementation ranging from sub-microseconds for small scale linearquadratic MPC to handling of strong nonlinearities, differential-algebraic dynamics and partial-differential equations in real-time feasible implementations. Several monographs provide overviews on the field of MPC, see (Ellis, Liu, et al., 2017; Grüne and Pannek, 2017) Rawlings and Mayne, 2009). In other words, nowadays MPC can be regarded as mature control method, which has had significant impact on industrial process control, cf. (Maciejowski, 2002, p. xi).

Standard control tasks frequently solved with NMPC include setpoint regulation and trajectory tracking, whereby the former refers to the stabilization of known setpoints defined in the state-space or some output space and the latter refers to the task of tracking time-dependent reference trajectories. However, even before first stability results on NMPC with state and inputs constraints were available, it has been observed by Morari et al., 1980 that

$$
\begin{aligned}
& \text { [in] attempting to synthesize a feedback optimizing control struc- } \\
& \text { ture, our main objective is to translate the economic objective into } \\
& \text { process control objectives. }
\end{aligned}
$$

The classical way to tackle this problem of designing economically beneficial control schemes is by means of the so-called control pyramid, wherein real-time optimization is used to compute economically desirable targets, which are then passed to the advanced process control, i.e. the MPC layer, (Engell, 2007). In other words, classically economic targets are translated into setpoints and reference trajectories, which are in

\footnotetext{
${ }^{1}$ In the literature, MPC often refers to the a setting with linear systems, convex quadratic objective and linear constraints while NMPC, which stands for nonlinear model predictive control, highlights the presence of nonlinear dynamics and non-convex constraints.
} 
turn stabilized by control techniques such as MPC. If indeed MPC is used to track these targets, then it is natural that the MPC objective penalizes mainly the deviation from the desired setpoint. It is this setting of setpoint regulation and tracking in which the vast majority of results on MPC stability and robustness of are formulated, cf. (Grüne and Pannek, 2017; Mayne et al., 2000; Rawlings and Mayne, 2009), and which is used frequently in industrial practice. At the same time, in process systems engineering and other fields of application, one aims at economic process operation. Hence, in the view of the quote from (Morari et al., 1980) given above, the question of closed-loop properties of receding-horizon optimal control with generic or economic objectives is very natural. In the process control community this issue has been addressed using the label Dynamic Real Time Optimization (Kadam and Marquardt, 2007), while in (Rawlings and Amrit, 2009) the term Economic Model Predictive Control (EMPC) has been coined.

The present survey provides a concise overview of different approaches on the question of stability and optimality in different formulations of EMPC. In contrast to previous overviews on the same topic (Ellis, Durand, et al., 2014), we cover approaches both with and without terminal constraints and end penalties, and turnpike/dissipativity-based settings as well as Lyapunov-based approaches.

\subsection{Outline}

In Section 2 we recall important stability results for stabilizing NMPC. Section 3 analyzes the stability of EMPC based on dissipativity properties and terminal constraints. Section 4 investigates the counterpart without terminal constraints and penalties. In Section 5 we provide an overview of performance bounds for the EMPC schemes from Section 3 and Section 4 .

EMPC with averaged constraints is discussed in Section 6, while Section 7 revisits generalized terminal constraints. Lyapunov-based approaches and multi-objective approaches are presented in Section 8, respectively, in Section 9. This survey ends with conclusions and an outlook on open issues in Section 10.

\subsection{Notation}

Throughout this review, we use the following notation: Real vectors are denoted by Latin letters, i.e. $x \in \mathbb{R}^{n_{x}}, u \in \mathbb{R}^{n_{u}}$. The two-norm of any vector $x \in \mathbb{R}^{n_{x}}$ is $\|x\|$.

Consider a discrete-time system $x(t+1)=f(x(t), u(t))$ with $f: \mathbb{R}^{n_{x}} \times \mathbb{R}^{n_{u}} \rightarrow \mathbb{R}^{n_{x}}$. 
The trajectory originating from $x_{0}$ driven by the input $u(\cdot)$ is written as $x\left(\cdot ; x_{0}, u(\cdot)\right)$. Whenever the control sequence is clear from context, we write simply $x\left(\cdot ; x_{0}\right)$.

We will use the following standard classes of comparison functions:

- $\mathcal{L}:=\left\{\gamma: \mathbb{R}_{0}^{+} \rightarrow \mathbb{R}_{0}^{+} \mid \gamma\right.$ continuous and decreasing with $\left.\lim _{k \rightarrow \infty} \gamma(k)=0\right\}$

- $\mathcal{K}:=\left\{\alpha: \mathbb{R}_{0}^{+} \rightarrow \mathbb{R}_{0}^{+} \mid \alpha\right.$ continuous and strictly increasing with $\alpha(0)=0\}$

- $\mathcal{K}_{\infty}:=\{\alpha \in \mathcal{K} \mid \alpha$ unbounded $\}$

- $\mathcal{K} \mathcal{L}:=\left\{\beta: \mathbb{R}_{0}^{+} \times \mathbb{R}_{0}^{+} \rightarrow \mathbb{R}_{0}^{+} \mid \beta(\cdot, k) \in \mathcal{K}, \beta(r, \cdot) \in \mathcal{L}\right\}$.

We refer to (Kellett, 2014) for a detailed overview of properties of these comparison functions. 



\section{Revisiting Stabilizing NMPC}

In this section, we give a brief introduction to the basic principle of MPC and recall available NMPC approaches for the classical control objective of (setpoint) stabilization. In NMPC, one repeatedly solves an Optimal Control Problem (OCP) in a receding horizon fashion, formulated either in a discrete or continuous time framework. In the present overview, we will focus mainly on the discrete-time framework, yet we will also comment on continuous-time counterparts of the presented results in various places. We begin with a concise review of NMPC for setpoint regulation.

\subsection{Main Idea of NMPC}

We consider autonomous discrete-time systems described by

$$
x(t+1)=f(x(t), u(t)), \quad x(0)=x_{0},
$$

where $x \in \mathbb{R}^{n_{x}}$ is the state, $u \in \mathbb{R}^{n_{u}}$ is the input and $f: \mathbb{R}^{n_{x}} \times \mathbb{R}^{n_{u}} \rightarrow \mathbb{R}^{n_{x}}$ denotes the continuous state transition map $!^{1}$ and $t \in \mathbb{Z}$ is the discrete time variable.

States and inputs are assumed to be restricted by the closed set $\mathbb{X} \subseteq \mathbb{R}^{n_{x}}$ and the compact set $\mathbb{U} \subset \mathbb{R}^{n_{u}}$, respectively. Both sets $\mathbb{X}$ and $\mathbb{U}$ contain the origin in their interior.

Corresponding to system (2.1), one considers a cost functional

$$
J_{\infty}\left(x_{0}, u(\cdot)\right)=\sum_{k=0}^{\infty} \ell(x(k), u(k))
$$

which models the performance requirements of 2.1 with the continuous stage cost $\ell: \mathbb{X} \times \mathbb{U} \rightarrow \mathbb{R}$.

Ultimately, one aims at optimizing the infinite-horizon objective $J_{\infty}$. However, this is numerically often infeasible. Thus, in NMPC one considers a finite horizon $N$ and the

\footnotetext{
${ }^{1}$ Note that many results in this survey extend to systems on general metric spaces, see (Grüne and Pannek, 2017).
} 
functional

$$
J_{N}\left(x_{0}, u(\cdot)\right)=\sum_{k=0}^{N} \ell(x(k), u(k))+V_{f}(x(N)),
$$

whereby the terminal penalty $V_{f}: \mathbb{X} \rightarrow \mathbb{R}$ is used to account for the truncation of the horizon. To this end, one initializes the closed loop system at $x(0)=x_{0}$ and solves the following finite-horizon discrete-time oCP at each time step $t=0,1,2, \ldots$ :

$$
\begin{array}{rlrl}
V_{N}(x(t)):=\min _{u(\cdot \mid t)} \sum_{k=0}^{N-1} \ell(x(k \mid t), u(k \mid t))+V_{f}(x(N \mid t)) & \\
\text { subject to } & & \\
x(k+1 \mid t) & =f(x(k \mid t), u(k \mid t)), & & k=0, \ldots, N-1 \\
x(0 \mid t) & =x(t) & & \\
(x(k \mid t), u(k \mid t))^{\top} & \in \mathbb{X} \times \mathbb{U}, & \\
x(N \mid t) & \in \mathbb{X}_{f} &
\end{array}
$$

With the resulting optimal input sequence denoted by $u^{\star}(k \mid t)$, one defines the MPC feedback as

$$
\mu_{N}(x(t)):=u^{\star}(0 \mid t)
$$

i.e., as the first element of the optimal input sequence, and obtains the next state of the closed loop system as

$$
x(t+1)=f\left(x(t), \mu_{N}(x(t))\right), \quad x(0)=x_{0} .
$$

Here, $N \in \mathbb{N}$ is the prediction horizon, $V_{f}: \mathbb{X} \rightarrow \mathbb{R}$ is the continuous terminal penalty, and $V_{N}(x(t))$ is the optimal value function of (2.4). Equations 2.4b $-2.4 \mathrm{~d}$ ) summarize the equality constraints imposed by the dynamics and additional constraints on states and inputs, which are typically described by inequalities. As we will recall shortly, the terminal constraint $2.4 \mathrm{e}$ is often used to enforce stability and recursive feasibility ${ }^{2}$ Here, $\mathbb{X}_{f} \subseteq \mathbb{X}$ is the terminal set or terminal region.

The superscript $(\cdot)^{\star}$ indicates variables related to optimal solutions of (2.4). Furthermore, in order to distinguish predicted variables from closed-loop variables, we use the

\footnotetext{
${ }^{2}$ Naturally, one may ask under which conditions does OCP (2.4) admit an optimal solution? As (2.4) is essentially a Nonlinear Program (NLP), we require continuity of $f, \ell$ and $V_{f}$. Note that our assumptions imply that an optimal solution to problem 2.4 exists in case the feasible set it not empty. For a further discussion on the existence of solutions of an NLP, the interested reader is referred to (Bertsekas, 1999).
} 
notation $\cdot(k \mid t)$ to denote $k$-step ahead predictions computed at time $t \in \mathbb{Z}$ based on the current (real) system state $x(t)$. For example, we write $u^{\star}(k \mid t)$ to refer to the $k$ th element of the optimal predicted input sequence to OCP (2.4) computed for the initial condition $x(t)$, and we denote the corresponding optimal state trajectory by $x^{\star}(\cdot \mid t)$. Throughout this survey we do not consider any plant-model mismatch, i.e., we assume that $f$ in (2.4b) and in 2.6) are identical.

As the feedback $\mu_{N}: \mathbb{X} \rightarrow \mathbb{U}$ from 2.5) relies on the receding-horizon solution of an optimization problem, it is necessary to discuss the feasibility properties of (2.4). To this end, we rely on the following definition:

Definition 2.1 (Recursive feasibility). Let $\mathbb{X}_{0} \subseteq \mathbb{X}$ denote a set of initial conditions $x(0)=x_{0}(2.4 \mathrm{c}$ for which $\mathrm{OCP}(2.4)$ admits a feasible solution. OCP (2.4) is said to be recursively feasible with respect to $\mathbb{X}_{0}$, if for all $x(0)=x_{0} \in \mathbb{X}_{0}$ the inclusion $f\left(x_{0}, \mu_{N}\left(x_{0}\right)\right) \in \mathbb{X}_{0}$ holds.

Now that we have stated the core idea of NMPC, several questions are immediate:

Q1 Under which conditions is OCP $(2.4)$ recursively feasible?

Q2 What are the properties of the closed-loop system 2.6 in terms of stability, optimality, and robustness?

Q3 Which stage costs $\ell$ are permissible without jeopardizing feasibility, stability, and optimality?

We will see later that questions Q1-Q3 are typically implicitly or explicitly answered in the course of analyzing any proposed NMPC scheme.

\subsection{Stabilizing NMPC with Terminal Constraints}

The classical problem to be tackled by NMPC is the stabilization of a given constant reference setpoint $\left(x_{s}, u_{s}\right) \in \operatorname{int}(\mathbb{X} \times \mathbb{U})$. Without loss of generality, we shift the target setpoint to the origin, i.e. we consider $\left(x_{s}, u_{s}\right)=(0,0)$ and $f\left(x_{s}, u_{s}\right)=0$. A typical design requirement in NMPC for setpoint regulation is that the stage cost $\ell$ penalizes the distance to the target $\left(x_{s}, u_{s}\right)=(0,0)$.

Assumption 2.1 (Lower boundedness of $\ell$ ). The stage cost satisfies $\ell(0,0)=0$. Furthermore, there exists $\alpha_{1} \in \mathcal{K}_{\infty}$ such that for all $(x, u) \in \mathbb{X} \times \mathbb{U}$

$$
\alpha_{1}(\|x\|) \leq \ell(x, u)
$$


In classical NMPC (Mayne et al., 2000; Rawlings and Mayne, 2009) one relies on the following key assumption to guarantee that $x=0$ is a stable equilibrium of the closedloop system (2.6) using the terminal penalty $V_{f}$ and the terminal constraint $\mathbb{X}_{f}$ :

Assumption 2.2 (Local bound on the cost-to-go). For all $x \in \mathbb{X}_{f}$, there exist an input $u=\kappa_{f}(x) \in \mathbb{U}$ such that $f\left(x, \kappa_{f}(x)\right) \in \mathbb{X}_{f}$ holds and

$$
V_{f}\left(f\left(x, \kappa_{f}(x)\right)\right)+\ell\left(x, \kappa_{f}(x)\right) \leq V_{f}(x)
$$

Furthermore, $V_{f}(0)=0$ and $V_{f}(x) \geq 0$ for all $x \in \mathbb{X}_{f}$.

An immediate consequence of Assumption 2.2 is that the terminal constraint $\mathbb{X}_{f}$ is a control invariant set, i.e., for any initial condition $x \in \mathbb{X}_{f}$, there exists a control input $u \in \mathbb{U}$ such that the successor state $x^{+}=f(x, u)$ satisfies $x^{+} \in \mathbb{X}_{f}$.

Now, we are ready to recall the well-known stability result for discrete-time stabilizing NMPC with terminal constraints.

Theorem 2.1 (Stability of NMPC with terminal constraints).

Let Assumptions 2.1 and 2.2 hold. Suppose that $0 \in \operatorname{int}\left(\mathbb{X}_{f}\right)$ and that there exists $\alpha_{3} \in \mathcal{K}_{\infty}$ such that, for all $x \in \mathbb{X}_{f}, V_{f}(x) \leq \alpha_{3}(\|x\|)$.

Then the closed-loop system (2.6) arising from the receding horizon solution to OCP (2.4) has the following properties:

(i) If OCP (2.4) is feasible for $t=0$, then it is feasible for all $t \in \mathbb{N}$.

(ii) The origin $x=0$ is an asymptotically stable equilibrium of (2.6).

(iii) The region of attraction of $x=0$ is given by the set of all initial conditions $x_{0}$ for which OCP 2.4 is feasible.

Proof. Throughout this overview we will only provide sketches of proofs and refer to the literature for details. The proof of Theorem 2.1 proceeds in two main steps: Step 1 establishes recursive feasibility, while Step 2 shows that the value function $V_{N}$ is a suitable candidate Lyapunov function of the closed-loop system.

Step 1: Consider ocP 2.4 for some initial condition $x(0 \mid t) \in \mathbb{X}$. Let $u^{\star}(\cdot \mid t)$ be the optimal input sequence and consider

$$
u(k \mid t+1)= \begin{cases}u^{\star}(k+1 \mid t), & k=0, \ldots, N-2 \\ \kappa_{f}\left(x^{\star}(N \mid t)\right), & k=N-1\end{cases}
$$


As we do not consider any plant-model mismatch, we have

$$
x(t+1)=f\left(x(t), u^{\star}(1 \mid t)\right)=x^{\star}(1 \mid t) \quad \in \mathbb{X} \quad \text { and } \quad x^{\star}(N \mid t) \in \mathbb{X}_{f}
$$

Thus, $u(\cdot \mid t+1)$ from (2.8) is feasible for ocP 2.4) with initial condition $x(t+1)=x^{\star}(1 \mid t)$.

Step 2: We use the value function $V_{N}$ from 2.4a as a Lyapunov function. By optimality of $V_{N}$ in (2.4) we have

$$
V_{N}(x(t+1))-V_{N}(x(t)) \leq J_{N}(x(t+1), u(\cdot \mid t+1))-V_{N}(x(t))
$$

where $J_{N}$, defined in 2.3$)$, is the finite-horizon counterpart of $J_{\infty}$, and $u(\cdot \mid t+1)$ is from (2.8). Since $u(k \mid t+1)=u^{\star}(k+1 \mid t), \quad k \in\{k, \ldots, N-2\}$ and $x(t+1)=x^{\star}(1 \mid t)$, we obtain

$$
\begin{aligned}
J_{N}(x(t+1), u(\cdot \mid t+1)) & -V_{N}(x(t)) \leq \\
-\alpha_{1}(\|x(t)\|)+ & \ell\left(x^{\star}(N \mid t), \kappa_{f}\left(x^{\star}(N \mid t)\right)\right) \\
& +V_{f}\left(f\left(x^{\star}(N \mid t), \kappa_{f}\left(x^{\star}(N \mid t)\right)\right)\right)-V_{f}\left(x^{\star}(N \mid t)\right)
\end{aligned}
$$

Taking Assumption 2.2 into account yields

$$
V_{N}(x(t+1))-V_{N}(x(t)) \leq-\alpha_{1}(\|x(t)\|) \leq 0,
$$

with $\alpha_{1} \in \mathcal{K}_{\infty}$ from Assumption 2.1. In other words, $V_{N}$ decreases strictly along closedloop trajectories. Without further elaboration, we note that one can also establish that $V_{N}$ is bounded from above by a suitable class $\mathcal{K}_{\infty}$ function on the set of all states where 2.4a is feasible, cf. (Rawlings and Mayne, 2009, Chap. 2). Hence, $V_{N}$ is a Lyapunov function of 2.6 on the set of all states where $2.4 \mathrm{a}$ is feasible.

Results similar to the one above appear in various forms in the literature: we refer to (Grüne and Pannek, 2017; Mayne et al., 2000; Rawlings and Mayne, 2009) for more detailed overviews of the literature. It is worth to be noted that Assumption 2.2 implies that the terminal penalty $V_{f}$ is an upper bound on the cost-to-go, i.e., for all $x \in \mathbb{X}_{f}$,

$$
V_{\infty}(x) \leq V_{f}(x)
$$

This has been observed in a continuous-time setting in (Chen and Allgöwer, 1998), wherein it is suggested to construct $X_{f}$ and $V_{f}$ by means of a linearization of (2.1) 
at $x=0, u=0$. The interesting special case, whereby the terminal set is a singleton $\mathbb{X}_{f}=\{0\}$, is commonly denoted as NMPC with zero terminal constraints. It dates back to (Keerthi and Gilbert, 1988; Michalska and Vinter, 1994) and gives rise to the following corollary:

Corollary 2.1 (Stability of NMPC with zero terminal constraints).

Let Assumption 2.1 hold, suppose that $V_{f}(x)=0$ and $\mathbb{X}_{f}=\{0\}$ are considered in OCP (2.4), and let $V_{N}$ be continuous at $x=0.3$ Then the closed-loop system (2.6) has the properties asserted in Theorem 2.1.

\subsection{Stabilizing NMPC without Terminal Constraints}

In the development of NMPC, the question for conditions ensuring that NMPC stabilizes a desired setpoint without consideration of terminal constraints has been thoroughly investigated. In general, one can distinguish three different approaches:

1. Replace $V_{f}$ by $V_{f}^{\beta}=\beta V_{f}$, with $\beta>0$ sufficiently large, such that a suitable terminal constraint $\mathbb{X}_{f}$ is satisfied without being explicitly stated in the ocP, cf. (Rawlings and Mayne, 2009).

2. Require that $V_{f}$ is a global Control Lyapunov Function (CLF) (Jadbabaie and Hauser, 2005).

3. Drop the terminal penalty $\left(V_{f}(x)=0\right)$, suppose specific bounds on the optimal value function, and require a sufficiently long prediction horizon (Grimm et al., 2005; Grüne, 2009; Jadbabaie, Yu, et al., 2001).

Approach 1 allows for inclusion of state constraints without jeopardizing recursive feasibility, as the arguments of Step 1 in the proof of Theorem 2.1 remain valid. However, it implicitly requires a preceding design of suitable terminal constraints.

Approach 2 is based on the observation that Assumption 2.2 can also be understood as the requirement of $V_{f}$ being a local CLF for (2.1). Hence, in Approach 2 one essentially requires Assumption 2.2 to hold for all $x \in \mathbb{X}$.

As we recall subsequently, Approach 3 allows showing that under suitable assumptions, for sufficiently long horizons $N$, NMPC is stabilizing. For the remainder of this section, we consider $\mathbb{X}_{f}=\mathbb{X}$ and $V_{f}(x)=0$.

\footnotetext{
${ }^{3}$ Requiring continuity of $V_{N}$ at $x=0$ is necessary since $0 \notin \operatorname{int}\left(\mathbb{X}_{f}\right)$.
} 
In Approach 2 and Approach 3 there is the underlying requirement that the state constraint set $\mathbb{X}$ is control invariant, which is often difficult to verify for nonlinear systems. To the end of avoiding recursive feasibility issues, we assume

Assumption 2.3 (X is control invariant). For each $x \in \mathbb{X}$ there exists $u \in \mathbb{U}$, such that $f(x, u) \in \mathbb{X}$.

Assumption 2.4 (Bound on $V_{N}(x)$ ). Consider OCP (2.4) with $\mathbb{X}_{f}=\mathbb{X}$ and $V_{f}(x)=0$. For each $x \in \mathbb{X}$, there exists $B_{K} \in \mathcal{K}_{\infty}, K \in \mathbb{N}$, such that

$$
V_{K}(x) \leq B_{K}\left(\ell^{*}(x)\right), \quad \text { with } \ell^{*}(x):=\inf _{u \in \mathbb{U}} \ell(x, u)
$$

holds for all $K \in \mathbb{N}$.

Theorem 2.2 (Stability of NMPC without terminal constraints).

Let Assumptions 2.1 and 2.3 hold. Suppose that Assumption 2.4 holds with $B_{K}(r)=$ $\gamma_{K} r, \sup _{k \in \mathbb{N}} \gamma_{k}<\infty$. Then, for sufficiently large $N$, the origin $x=0$ is an asymptotically stable equilibrium of the closed-loop system (2.6).

Proof. The above result appears as Theorem 6.24 in (Grüne and Pannek, 2017). Its proof is centered around the relaxed dynamic programming inequality

$$
V_{N}\left(f\left(x, \mu_{N}(x)\right) \leq V_{N}(x)-\alpha \ell\left(x, \mu_{N}(x)\right)\right.
$$

for $\alpha \in(0,1]$, which implies both asymptotic stability of 2.6 with $V_{N}$ as a Lyapunov function and the suboptimality estimate

$$
J_{\infty}\left(x, \mu_{N}(\cdot)\right) \leq \frac{1}{\alpha} V_{N}(x) \leq \frac{1}{\alpha} V_{\infty}(x)
$$

cf. Theorem 4.11 from (Grüne and Pannek, 2017).

The proof proceeds by showing that the linearity assumption on $B_{K}$ implies that, for $N \rightarrow \infty$, there exists an appropriate $\alpha \in(0,1]$ that satisfies 2.10 for all $x \in \mathbb{X}$, cf. (Grüne and Pannek, 2017, Prop. 6.18 and Thm. 6.24).

We refer to (Grüne and Pannek, 2017, Chap. 6) for details and a discussion of Assumption 2.4. We also note that if Assumption 2.4 holds with nonlinear functions $B_{K}$,

\footnotetext{
${ }^{4}$ Note that this assumption can be relaxed, see (Grüne and Pannek, 2017, Chap. 7). Furthermore, in Section 4 we investigate relaxing this assumption in the context of EMPC.
} 
then one can still show semiglobal practical asymptotic stability w.r.t. the prediction horizon N, see (Grüne and Pannek, 2017, Thm. 6.37).

In summary, the design of MPC schemes for stabilization problems is by now well understood and various different NMPC approaches exist in the literature to this end, see (Grüne and Pannek, 2017; Rawlings and Mayne, 2009) for detailed overviews. We conclude our brief discussion of stabilizing NMPC by commenting on the advantages and disadvantages of NMPC schemes with and without terminal constraints and/or penalty.

The main advantages of NMPC schemes using terminal constraints include the following: (i) A shorter prediction horizon might be sufficient for closed-loop stability than in a setting without terminal constraints. (ii) A systematic procedure how to satisfy the crucial Assumption 2.2 is available for a large class of systems (in particular, in case that the desired equilibrium is contained in the interior of the state constraints and the linearization at this point is stabilizable). On the other hand, the addition of terminal constraints might be restrictive and can result in an (unnecessarily) small feasible set (depending on the size of the terminal region and on the length of the prediction horizon). Also, the additional terminal constraints result in an increased computational complexity.

At the same time, in many applications NMPC is often implemented without any terminal constraint. The main advantages of doing so are that (i) omitting terminal constraints results in a simpler optimization problem and (ii) typically a larger feasible set is obtained. On the other hand, establishing recursive feasibility of the OCP is not as straightforward but requires additional assumptions/arguments. Furthermore, the crucial controllability assumption (Assumption 2.4 might be difficult to verify, and no systematic procedure exists to this end for general nonlinear systems. In conclusion, each of the presented schemes has its advantages and disadvantages, which have to be considered when choosing a suitable NMPC scheme for a given stabilization task. 


\section{Economic MPC with Terminal Constraints}

As already mentioned in the introduction, in many applications achieving a reasonable trade-off between safety, i.e. stability, and economic process operation is of key importance. In this context, it has been, and still is, common practice in industry to translate economic operation into desired target setpoints, which can then be stabilized and tracked, for instance, by means of the NMPC schemes sketched in Section 2. Moreover, by now powerful numerical methods for implementation of NMPC for large-scale process control applications, fast mechatronic systems, and other domains are available. As we have seen in the previous section, there also exists a mature body of theory on stability/optimality/robustness of NMPC relying on the classical boundedness of the stage cost $\ell$ by a distance measure (Assumption 2.1).

However, it is quite natural to consider feedback schemes built around receding horizon optimal control using generic stage costs $\ell$, i.e. to consider NMPC for given functions $\ell$ instead of NMPC with $\ell$ being designed to the end of tracking target setpoints. In the view of Question Q3 stated in Section 2, which asks for permissible stage costs $\ell$, we now turn the discussion towards replacing Assumption 2.1 with weaker properties in the NMPC stability analysis. To this end and for the remainder of this section, we consider NMPC based on the receding horizon solution to the following OCP with terminal constraint:

$$
\begin{array}{rlrl}
V_{N}(x(t)):=\min _{u(\cdot \mid t)} \sum_{k=0}^{N-1} \ell(x(k \mid t), u(k \mid t))+V_{f}(x(N \mid t)) & \\
\text { subject to } & & \\
x(k+1 \mid t) & =f(x(k \mid t), u(k \mid t)), & & k=0, \ldots, N-1 \\
x(0 \mid t) & =x(t) & & k=0, \ldots, N-1 \\
(x(k \mid t), u(k \mid t))^{\top} & \in \mathbb{X} \times \mathbb{U}, & \\
x(N \mid t) & \in \mathbb{X}_{f} &
\end{array}
$$




\subsection{Dissipativity and Optimal Operation at Steady State}

We begin with relaxing Assumption 2.1 to a dissipativity notion, which appears to have been made first in (Angeli et al., 2012; Diehl et al., 2011).

Definition 3.1 (Strict dissipativity with respect to a steady state).

System (2.1) is said to be dissipative with respect to the steady-state pair $\left(x_{s}, u_{s}\right) \in$ $\mathbb{X} \times \mathbb{U}$, if there exists a non-negative function $\lambda: \mathbb{X} \rightarrow \mathbb{R}$ such that for all $x \in \mathbb{X}, u \in \mathbb{U}$

$$
\lambda(f(x, u))-\lambda(x) \leq \ell(x, u)-\ell\left(x_{s}, u_{s}\right) .
$$

If, additionally, there exists $\alpha_{\ell} \in \mathcal{K}_{\infty}$ such that

$$
\lambda(f(x, u))-\lambda(x) \leq-\alpha_{\ell}\left(\left\|\left(x-x_{s}, u-u_{s}\right)\right\|\right)+\ell(x, u)-\ell\left(x_{s}, u_{s}\right) .
$$

then (2.1) is said to be strictly dissipative with respect to $\left(x_{s}, u_{s}\right)$.

We remark that $\ell$ in 3.2 refers to the stage cost of OCP (3.1). Denoting

$$
s(x, u)=\ell(x, u)-\ell\left(x_{s}, u_{s}\right)
$$

as a supply rate and calling $\lambda$ in (3.2) a storage function, it is clear that (3.2) are dissipation inequalities ${ }^{1}$

Remark 3.1 (Different dissipation inequalities considered in EMPC).

We remark that the dissipation inequalities (3.2) appear in different variants in the EMPC literature: While (Diehl et al., 2011) suggest linear storage functions, (Angeli et al., 2012; Damm et al., 2014; Faulwasser and Bonvin, 2015b; Grüne, 2013) consider nonlinear functions $\lambda: \mathbb{X} \rightarrow \mathbb{R}$. Moreover, some results in the context of EMPC are also valid when using storage functions which are not necessarily bounded from below? such as, e.g., Theorem 3.2 below (without loss of generality boundedness from below is equivalent to non-negativity as typically assumed in classical dissipativity theory (Willems, 1972)). Furthermore, some results not only require boundedness from below of the storage function $\lambda$, but also boundedness from above (i.e., a bounded storage function $\lambda$ ).

\footnotetext{
${ }^{1}$ It is worth to be noted that dissipation inequalities can be used to analyze different system properties ranging from stability to non-minimum phase behavior (Ebenbauer et al., 2009). However, an indepth introduction to dissipativity concepts in systems theory is beyond the scope of the present survey. Instead we refer to (Moylan, 2014, Willems, 2007; Willems, 1972).

${ }^{2}$ In the classical dissipativity literature, this is referred to as cyclo-dissipativity see (Hill and Moylan, 1980, Moylan, 2014).
} 
This is, e.g., the case in Section 4 or in converse dissipativity (Müller, Angeli, and Allgöwer, 2015) and converse turnpike results (Faulwasser et al., 2014, 2017; Grüne and Müller, 2016). Finally, we remark that while most of the available EMPC results require strictness in (3.2b) with respect to $x-x_{s}$, for some results strictness in $x$ and $u$ is required, such as in the converse turnpike results presented in (Faulwasser et al., 2014, 2017), when considering robustness of dissipativity (Müller, Angeli, and Allgöwer, 2015), or in the more general case of optimal periodic operation (Müller and Grüne, 2016a). Henceforth, as it simplifies some of our later developments, we consider strictness in $x$ and $u$.

In Definition 3.1, the dissipation inequalities are required to hold on $\mathbb{X} \times \mathbb{U}$. On the other hand, most of the results in EMPC also hold if these inequalities are only satisfied on a certain subset of $\mathbb{X} \times \mathbb{U}$. For example, in (Müller, Grüne, and Allgöwer, 2015; Müller, Angeli, and Allgöwer, 2015) all $(x, u)$ pairs belonging to an infinite-horizon feasible trajectory are considered, while (Faulwasser and Bonvin, 2015b; Faulwasser et al., 2017) employ dissipativity along optimal solutions of OCP (3.1) in the following sense:

Definition 3.2 (Strict dissipativity of ocP (3.1)). If, for all $N \in \mathbb{N}$ and all $x_{0} \in \mathbb{X}_{0}$, the dissipation inequalities (3.2) hold along any optimal pair of OCP (3.1), then OCP (3.1) is said to be (strictly) dissipative.

Observe that in the non-strict case, Definition 3.2 and Definition 3.1 are equivalent 3 However, in the strict case Definition 3.2 is weaker than the dissipativity property required in Definition 3.1. As noted above, the majority of the available EMPC results have been formulated using Definition 3.1, however, most of them can also be shown using Definition 3.2 instead. If the latter dissipativity definition is employed, the resulting closed-loop guarantees are only valid under the assumption that the optimal solution to OCP 3.1 can be found online. However, from an applications point of view, it is evident that one often computes only approximately optimal solutions to OCP (3.1). From this perspective, we note without further elaboration that the stronger dissipativity notion of Definition 3.1 implies a certain robustness with respect to the application of suboptimal feedbacks in NMPC.

The dissipativity notions introduced above are of importance as they establish a relation between the trajectories of system (2.1) and the stage cost of oCP (3.1).

Lemma 3.1 (Dissipativity and steady-state optimality). If system (2.1) is dissipative

\footnotetext{
${ }^{3}$ This directly follows from Theorem 1 in (Willems, 1972).
} 
with respect to $\left(x_{s}, u_{s}\right) \in \mathbb{X} \times \mathbb{U}$, then $\left(x_{s}, u_{s}\right)$ in $3.2 \mathrm{~b}$ is a global minimizer of

$$
\min _{(x, u)} \ell(x, u)
$$

subject to

$$
x=f(x, u) \quad \text { and } \quad(x, u) \in \mathbb{X} \times \mathbb{U} .
$$

If, moreover, system (2.1) is strictly dissipative with respect to $\left(x_{s}, u_{s}\right)$, then $\left(x_{s}, u_{s}\right)$ is the unique global minimizer of (3.4).

Proof. For sake of contradiction, suppose that $\left(x_{s}, u_{s}\right)$ is not a global minimizer of (3.4). Then, there exists a steady-state pair $(\bar{x}, \bar{u})$ such that $\ell(\bar{x}, \bar{u})-\ell\left(x_{s}, u_{s}\right)<0$. Evaluating the dissipation inequality (3.2a at $(\bar{x}, \bar{u})$ gives $0 \leq \ell(\bar{x}, \bar{u})-\ell\left(x_{s}, u_{s}\right)$. Hence, unless $\ell(\bar{x}, \bar{u})=\ell\left(x_{s}, u_{s}\right)$ for all minimizers of (3.4), we arrive at a contradiction.

Evaluating the strict dissipation inequality $(3.2 \mathrm{~b})$ at $(\bar{x}, \bar{u})$ gives, for all steady states $\bar{x} \neq x_{s}, 0<\alpha_{\ell}\left(\left\|\left(\bar{x}-x_{s}, \bar{u}-u_{s}\right)\right\|\right) \leq \ell(\bar{x}, \bar{u})-\ell\left(x_{s}, u_{s}\right)$. Hence, the pair $\left(x_{s}, u_{s}\right)$ is the unique global minimizer of 3.4 . .

The reader may interpret the strict dissipativity property of Definition 3.1 as the relaxation of Assumption 2.1 in the sense that the lower boundedness of $\ell$ by a distance measure is only required on the set of steady states of (2.1).

We will show in Section 4 that dissipativity of an OCP also allows statements about the specific structure of its optimal solutions, i.e. we will link it to turnpike properties. Besides, it allows to make qualitative statements about how to operate a process optimally on infinite horizons. In particular, one can show that dissipativity of system (2.1) as in Definition 3.1 implies that the system is optimally operated at steady state, which is formally defined as follows:

Definition 3.3 (Optimal steady-state operation). The system 2.1 is optimally operated at steady-state, if for each solution satisfying $(x(t), u(t)) \in \mathbb{X} \times \mathbb{U}$ for all $t \in \mathbb{N}$ the following holds:

$$
\liminf _{T \rightarrow \infty} \frac{\sum_{t=0}^{T} \ell(x(t), u(t))}{T+1} \geq \ell\left(x_{s}, u_{s}\right) .
$$

Definition 3.3 means that no feasible input and state sequence pair results in a better asymptotic average performance than the optimal steady-state cost. The following result showing sufficiency of dissipativity for optimal steady-state operation was obtained in (Angeli et al., 2012): 
Theorem 3.1 (Dissipativity implies optimal steady-state operation).

Suppose that system (2.1) is dissipative with respect to the steady-state pair $\left(x_{s}, u_{s}\right)$. Then it is optimally operated at steady state.

The proof of this result follows in a straightforward fashion from the dissipation inequality (3.2a), by noting that

$$
\begin{aligned}
0 & \leq \liminf _{T \rightarrow \infty} \frac{\lambda(x(T))-\lambda(x(0))}{T}=\liminf _{T \rightarrow \infty} \frac{\sum_{t=0}^{T-1} \lambda(f(x(t), u(t)))-\lambda(x(t))}{T} \\
& \leq \liminf _{T \rightarrow \infty} \frac{\sum_{t=0}^{T-1} \ell(x(t), u(t))-\ell\left(x_{s}, u_{s}\right)}{T} \\
& =\liminf _{T \rightarrow \infty} \frac{\sum_{t=0}^{T-1} \ell(x(t), u(t))}{T}-\ell\left(x_{s}, u_{s}\right),
\end{aligned}
$$

which is 3.5 .

As shown in (Müller, Angeli, and Allgöwer, 2015), under a suitable controllability condition, the converse statement is also true, i.e., dissipativity is also necessary for optimal steady-state operation. Furthermore, strict dissipativity with respect to the steady-state pair $\left(x_{s}, u_{s}\right)$ can be used as a sufficient and necessary condition (the latter again under a suitable local controllability assumption) for a slightly stronger property than optimal steady state operation, termed uniform suboptimal operation off steady state (see Müller, Grüne, and Allgöwer, 2015), meaning that steady-state operation is the unique optimal operating behavior in a suitable sense.

In summary, dissipativity with respect to the steady-state pair $\left(x_{s}, u_{s}\right)$ serves as an (almost) equivalent characterization for the fact that the optimal (infinite horizon) operating behavior of system $(2.1)$ is steady-state operation at $\left(x_{s}, u_{s}\right)$.

\subsection{Closed-loop Stability}

In case that system (2.1) is optimally operated at steady-state, a well defined economic MPC scheme should ensure that the closed loop indeed converges to the optimal steady state $x_{s}$. In the following, we show that this is indeed the case. To this end, it has been suggested in (Amrit et al., 2011; Angeli et al., 2012; Diehl et al., 2011) to consider the following rotation of the stage cost $\ell$ and the end penalty $V_{f}$

$$
\begin{aligned}
\tilde{\ell}(x, u) & =\ell(x, u)-\ell\left(x_{s}, u_{s}\right)+\lambda(x)-\lambda(f(x, u)), \\
\widetilde{V}_{f}(x) & =V_{f}(x)+\lambda(x)
\end{aligned}
$$


It is readily seen that $\tilde{\ell}$ is lower bounded by a suitable class $\mathcal{K}$ function if the strict dissipation inequality $3.2 \mathrm{~b}$ is satisfied. Additionally, it turns out that the solutions to OCP (3.1) are not affected swapping $\ell$ with $\widetilde{\ell}$ and $V_{f}$ with $\widetilde{V}_{f}$.

Lemma 3.2 (Rotation does not change optimal solutions).

Consider any horizon $N \in \mathbb{N}$ and any initial condition $x$ such that OCP (3.1) with stage cost $\ell$ and terminal penalty $V_{f}$ admits an optimal solution $u^{\star}(\cdot)$.

Then, for the same horizon $N \in \mathbb{N}$ and initial condition $x$, the input sequence $u^{\star}(\cdot)$ is also optimal in OCP (3.1) employing the rotated costs $\tilde{\ell}$ and $\widetilde{V}_{f}$.

Proof. Consider an admissible pair $x(\cdot), u(\cdot)$ defined for some horizon $N \in \mathbb{N}$, originating at some initial conditions $x$. Simple calculations show that

$$
\sum_{k=0}^{N-1} \ell(x(k), u(k))-\tilde{\ell}(x(k), u(k))=\lambda(x(N))-\lambda(x)+N \cdot \ell\left(x_{s}, u_{s}\right)
$$

Since $V_{f}(x(N))-\widetilde{V}_{f}(x(N))=-\lambda(x(N))$, for any admissible pair, the objectives of oCP (3.1) with costs $l, V_{f}$ and ocP 3.1 considering the rotated costs $\tilde{\ell}, \widetilde{V}_{f}$ differ only by the constant $-\lambda(x)+N \cdot \ell\left(x_{s}, u_{s}\right)$.

We note that the last result does not require that $\lambda$ satisfies any dissipation inequality. Hence, rotation by any bounded function does not change the optimal solution in OCP (3.1). Now we are ready to state the EMPC counterpart of Theorem 2.1.

Theorem 3.2 (Stability of EMPC with terminal constraints). Suppose that system (2.1) is strictly dissipative with respect to the steady-state pair $\left(x_{s}, u_{s}\right)$ Furthermore, let Assumption 2.2 hold for $\widetilde{V}_{f}$ from (3.6) and suppose that $x_{s} \in \operatorname{int}\left(\mathbb{X}_{f}\right)$.

Then the closed-loop system (2.6) arising from the receding horizon solution to OCP (3.1) has the following properties:

(i) If OCP (3.1) is feasible for $k=0$, then it is feasible for all $k \in \mathbb{N}$.

(ii) The steady state $x=x_{s}$ is an asymptotically stable equilibrium of (2.6).

(iii) The region of attraction of $x=x_{s}$ is given by the set of all initial conditions $x_{0}$ for which OCP (3.1) is feasible.

Proof. Since the optimal solutions coincide, we may consider OCP (3.1) using the rotated costs from (3.6), cf. Lemma 3.2. Since the system (2.1) is strictly dissipative with respect to the steady-state pair $\left(x_{s}, u_{s}\right)$, inequality $3.6 \mathrm{a}$ is satisfied. Hence, all conditions of Theorem 2.1 are satisfied. 
Similar to Corollary 2.1 by enforcing a point-wise terminal constraint we immediately obtain the following result:

Corollary 3.1 (Stability of EMPC with zero terminal constraints). Suppose that that system (2.1) is strictly dissipative with respect to the steady-state pair $\left(x_{s}, u_{s}\right)$. Moreover, consider the terminal constraint set $\mathbb{X}_{f}=\left\{x_{s}\right\}$ and let $\lambda$ and $V_{N}$ be continuous at $x=x_{s}$.

Then the closed-loop system (2.6) arising from the receding horizon solution to OCP (3.1) has the properties asserted in Theorem 3.2.

We remark that the original version of Theorem 3.2 was presented in (Amrit et al., 2011), while Corollary 3.1 was presented in (Angeli et al., 2012, Thm. 2), where strict dissipativity is required only with respect to $x_{s}$. Furthermore, the results of (Diehl et al., 2011) represent Corollary 3.1 for the case of linear storage functions $\lambda$. A continuoustime extension can be found in (Alessandretti et al., 2014).

Combining the above results with those of Section 3.1, we obtain the following conclusions on EMPC with terminal constraints: If the optimal operating behavior for system (2.1) is steady-state operation (in its strict form), the system is (strictly) dissipative with respect to the steady-state pair $\left(x_{s}, u_{s}\right)$, which in turn implies asymptotic stability of $x_{s}$ for the resulting closed-loop system. Put differently, this means that the closed-loop system "finds" the optimal operating behavior (compare (Müller, Grüne, and Allgöwer, 2015 for a more detailed discussion on these issues). Notably, these conclusions can be drawn without the explicit knowledge of a storage function $\lambda$, which is important since computing such a storage function can be difficult for general nonlinear systems and cost functions. However, the optimal steady state $x_{s}$ needs to be known a priori, since the asymptotic stability properties only hold if the terminal constraint $3.1 \mathrm{e}$ and terminal penalty $V_{f}$ are specified with respect to the optimal steady state $x_{s}$.

\subsection{Example - Chemical Reactor with Dissipativity}

To illustrate the asymptotic stability result, we consider the Van de Vusse reactor as an example, see (Klatt et al., 1995; Rothfuß et al., 1996). In a continuously stirred tank reactor, three endothermal chemical reactions $A \stackrel{k_{1}}{\longrightarrow} B \stackrel{k_{2}}{\longrightarrow} C$ and $2 A \stackrel{k_{3}}{\longrightarrow} D$ take place. A partial model of the reactor, including the concentration of species $A$ and $B, c_{A}, c_{B}$ 
in $\mathrm{mol} / \mathrm{l}$ and the reactor temperature $\vartheta$ in ${ }^{\circ} \mathrm{C}$ as state variables, reads

$$
\begin{aligned}
\dot{c}_{A} & =r_{A}\left(c_{A}, \vartheta\right)+\left(c_{i n}-c_{A}\right) u_{1} \\
\dot{c}_{B} & =r_{B}\left(c_{A}, c_{B}, \vartheta\right)-c_{B} u_{1} \\
\dot{\vartheta} & =h\left(c_{A}, c_{B}, \vartheta\right)+\alpha\left(u_{2}-\vartheta\right)+\left(\vartheta_{i n}-\vartheta\right) u_{1},
\end{aligned}
$$

where

$$
\begin{aligned}
r_{A}\left(c_{A}, \vartheta\right)= & -k_{1}(\vartheta) c_{A}-2 k_{3}(\vartheta) c_{A}^{2} \\
r_{B}\left(c_{A}, c_{B}, \vartheta\right)= & k_{1}(\vartheta) c_{A}-k_{2}(\vartheta) c_{B} \\
h\left(c_{A}, c_{B}, \vartheta\right)= & -\delta\left(k_{1}(\vartheta) c_{A} \Delta H_{A B}+k_{2}(\vartheta) c_{B} \Delta H_{B C}\right. \\
& \left.\quad+2 k_{3}(\vartheta) c_{A}^{2} \Delta H_{A D}\right) \\
k_{i}(\vartheta)= & k_{i 0} \exp \frac{-E_{i}}{\vartheta+\vartheta_{0}}, \quad i=1,2,3 .
\end{aligned}
$$

The system parameters can be found in (Rothfuß et al., 1996). The inputs $u_{1}, u_{2}$ are the normalized flow rate of $A$ through the reactor in $1 / \mathrm{h}$ and the temperature in the cooling jacket in ${ }^{\circ} \mathrm{C}$. The states and inputs are subject to the constraints

$$
\begin{array}{lll}
c_{A} \in[0,6] \frac{\mathrm{mol}}{l} & c_{B} \in[0,4] \frac{\mathrm{mol}}{\mathrm{l}} & \vartheta \in[70,200]^{\circ} \mathrm{C} \\
u_{1} \in[3,35] \frac{1}{h} & u_{2} \in[0,200]^{\circ} \mathrm{C} . &
\end{array}
$$

We consider the problem of maximizing the production rate of $c_{B}$; thus we specify the cost function $L$ as

$$
L\left(c_{B}, u_{1}\right)=-c_{B} u_{1}
$$

As shown in (Faulwasser et al., 2017), the system is strictly dissipative at the optimal steady-state pair

$$
x_{s}=(2.175,1.105,1.285)^{\top} \quad u_{s}=1.428
$$

in appropriately rescaled variables (see the next paragraph) 4 Moreover, the constrained reachability properties of the system have been analyzed in (Faulwasser, Hagenmeyer, et al., 2014).

In order to convert the system into a discrete time system, we fix the sampling rate as 0.0033 and use a numerical approximation of the solutions of the differential equation by

\footnotetext{
${ }^{4}$ We remark that, to the end computing a storage function via sum-of-squares programming, Faulwasser et al., 2017 use a polynomial approximation of the exponential terms in $k_{i}(\vartheta)$. Hence, the globally optimal steady state considered in (Faulwasser et al., 2017) slightly differs.
} 

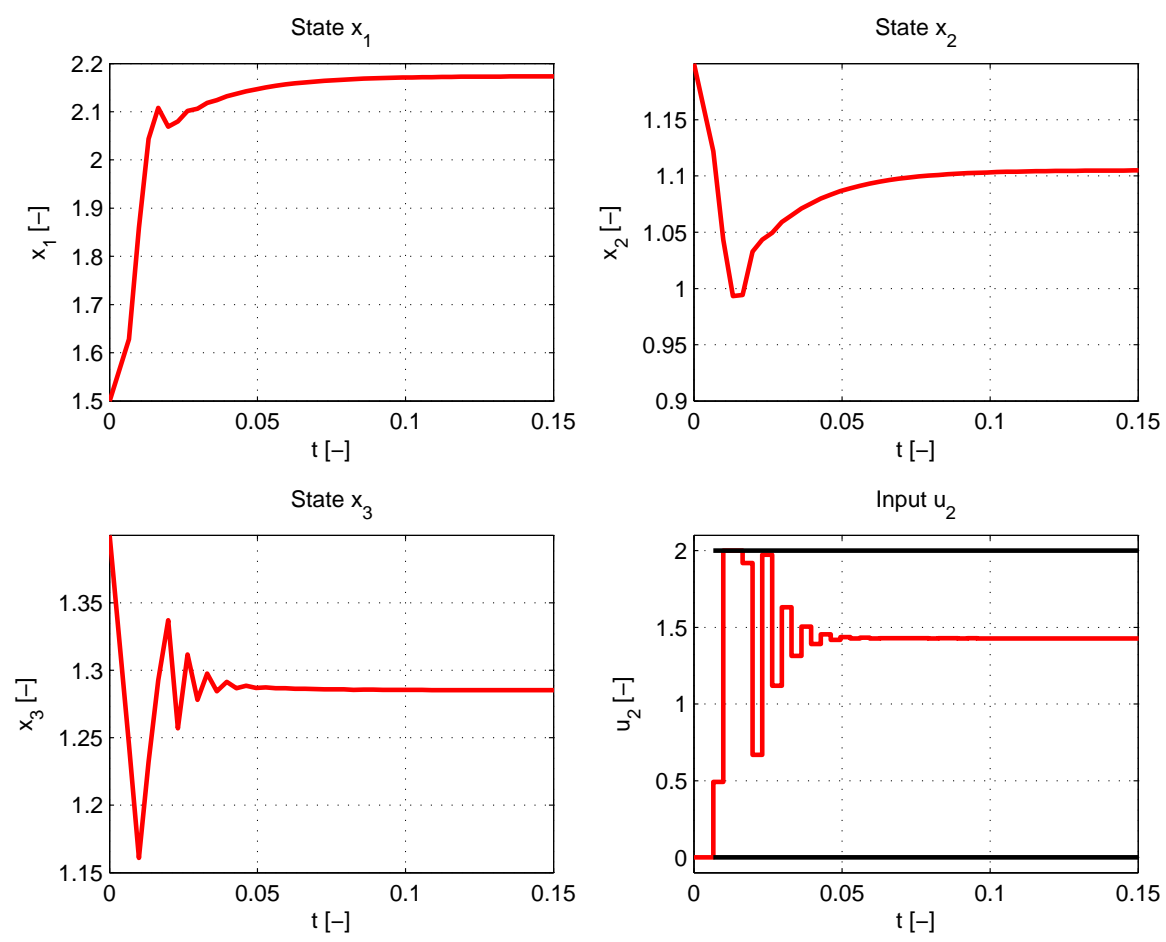

Figure 3.1: EMPC closed loop solution for the Van de Vusse reactor with terminal constraints $x(N)=x_{s}, N=20$.

means of an embedded Runge-Kutta scheme of order $8(7)$. The stage cost $\ell$ is defined as the integral over $L$ along the solution over one sampling interval. The three states of the discretized model will be denoted by $x_{i}, i=1,2,3$. The resulting OCP is solved with an open-source direct multiple shooting implementation available in ACADO, see (Houska, Ferreau, et al., 2011). In order to improve numerical stability, the states, the inputs and the cost function were appropriately re-scaled in this implementation. Particularly, $u_{2}$ and $\vartheta=x_{3}$ were rescaled by $10^{-2}$, thus the respective constraint sets become $[0,2]$ and $[0.7,2]$. In the following figures we only show the input $u_{2}$ because $u_{1}$ is constantly equal to the upper boundary of the input constraint set. All solutions were started from the initial value $x_{0}=(1.5,1.2,1.4)^{\top}$.

We first show the state trajectories with terminal equality constraint $x(N \mid t)=x_{s}$. As Figure 3.1 shows, the solutions, here for horizon $N=20$, converge to the optimal equilibrium, as expected. The oscillations are due to numerical instabilities which are presumably caused by the terminal constraints. As we will see in Section 4.4, they will be reduced in MPC without terminal constraints. 


\subsection{Example - Chemical Reactor without Dissipativity}

In order to show that without strict dissipativity asymptotic stability may not hold, we consider an example taken from (Bailey et al., 1971), which has also been considered in (Müller, Angeli, Allgöwer, et al., 2014). In a continuous chemical reactor the following parallel reaction scheme $R \longrightarrow P_{1}, R \longrightarrow P_{2}$ takes place, whereby the component $R$ is converted into the desired product $P_{1}$ and the waste product $P_{2}$. Assuming that the reactions are isothermal, the dimensionless heat and (partial) mass balance of this reaction scheme leads to the following dynamic model

$$
\begin{aligned}
& \dot{x}_{1}=1+r_{1}\left(x_{1}, x_{3}\right)-x_{1} \\
& \dot{x}_{2}=r_{2}\left(x_{1}, x_{3}\right)-x_{2} \\
& \dot{x}_{3}=u-x_{3}
\end{aligned}
$$

where $r_{1}: \mathbb{R}^{2} \rightarrow \mathbb{R}$ and $r_{2}: \mathbb{R}^{2} \rightarrow \mathbb{R}$ are

$$
r_{1}\left(x_{1}, x_{3}\right)=-10^{4} x_{1}^{2} e^{-\frac{1}{x_{3}}}-400 x_{1} e^{-\frac{0.55}{x_{3}}} \text { and } r_{2}\left(x_{1}, x_{3}\right)=400 x_{1} e^{-\frac{0.55}{x_{3}}}
$$

The state $x_{1}$ models the concentration of $R$; the state $x_{2}$ models the concentration of the desired product $P_{1}$; the state $x_{3}$ is the dimensionless temperature of the mixture in the reactor; and the input $u$ is related to the heat flux through the cooling jacket. The state and input constraints are

$$
x_{i} \geq 0, i=1,2,3 \quad \text { and } \quad u \in[0.049,0.449] .
$$

The objective is maximizing the amount of product $P_{1}$, i.e. the objective is the integral over

$$
L(x, u)=-x_{2}
$$




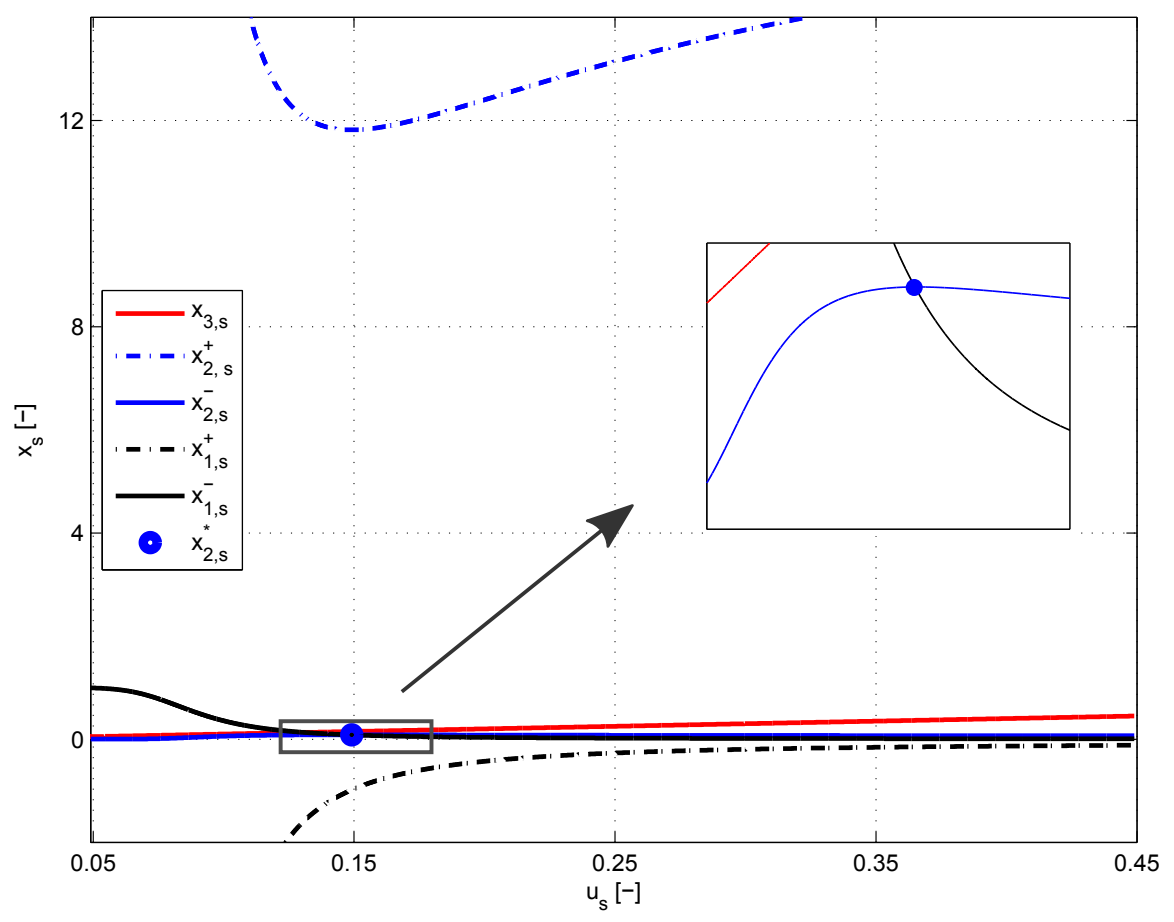

Figure 3.2: Steady states of (3.10): Dashed curves refer to the case of + in $(3.12 a)$ and continuous curves refer to the case of - .

\section{Steady State Analysis}

Simple calculations show that the steady states of $(3.10)$ are given by

$$
\begin{aligned}
& x_{1, s}=\frac{\left(1+400 e^{-\frac{0.55}{x_{3, s}}}\right) \pm \sqrt{\left(1+400 e^{-\frac{0.55}{x_{3, s}}}\right)^{2}+4 \cdot 10^{4} e^{-\frac{1}{x_{3, s}}}}}{-2 \cdot 10^{4} e^{-\frac{1}{x_{3, s}}}} \\
& x_{2, s}=-r_{2}\left(x_{1, s}, x_{3, s}\right) \\
& x_{3, s}=u_{s} .
\end{aligned}
$$

Observe that due to the identity $x_{3, s}=u_{s}$, one can parametrize the steady states of (3.10) by $u_{s}$. The corresponding solutions are illustrated in Figure 3.2. As we are interested in chemically meaningful steady states, all dashed solutions are neglected. Using (3.12) the globally optimal steady state pair is easily found as

$$
x_{s}=(0.0832,0.0846,0.1491)^{\top} \quad u_{s}=0.1491 \text {. }
$$



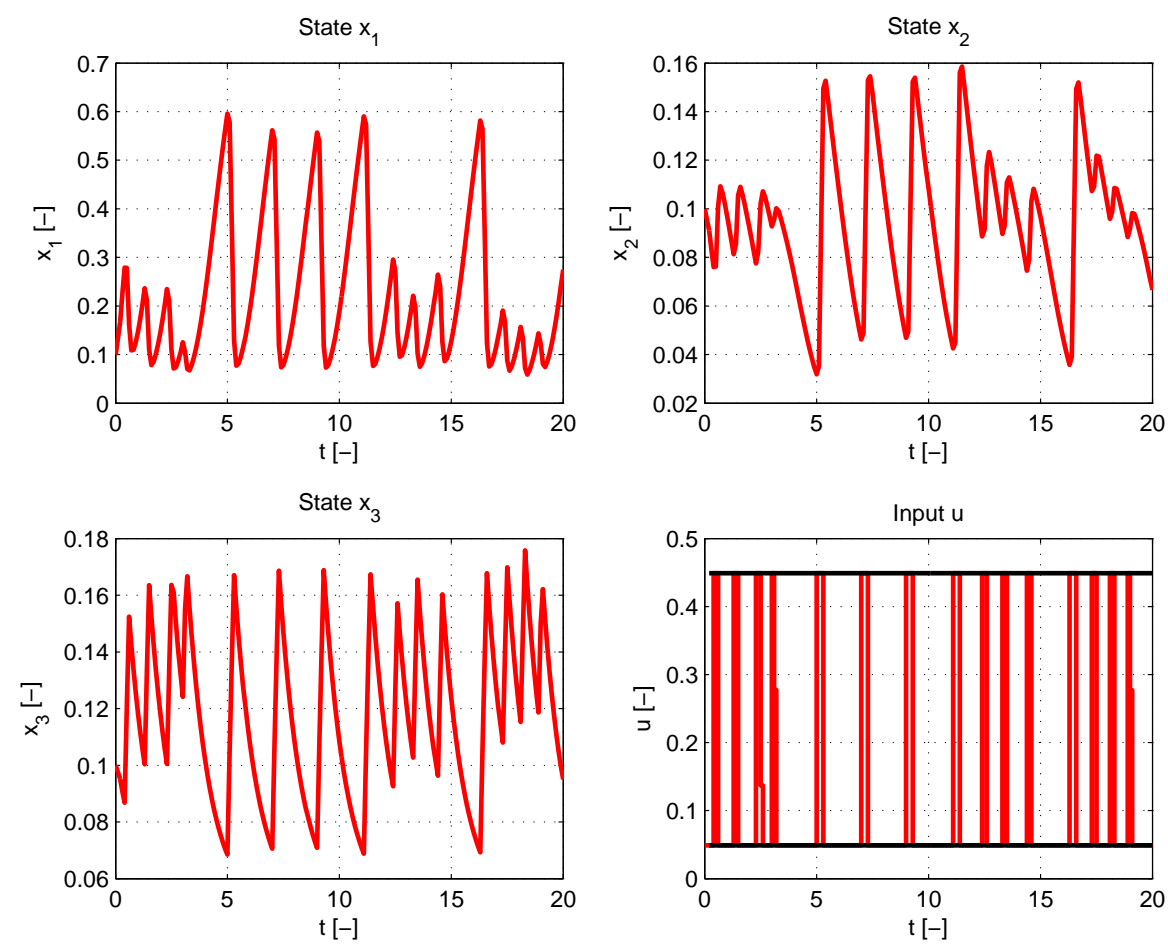

Figure 3.3: Closed-loop EMPC with zero terminal constraint and $L(x, u)=-x_{2}$.

\section{Closed-loop EMPC}

We consider EMPC based on OCP (3.1) with the data from (3.10) and 3.11). We solve the OCP by means of the open-source direct multiple-shooting implementation in ACADO (Houska, Ferreau, et al., 2011) using a Runge-Kutta scheme of order 5(4) for integration. We employ a piecewise constant input parametrization with a sampling period of 0.1 . The prediction horizon is set to 5 time units, i.e. we have $N=50$. The terminal constraint $3.1 \mathrm{e}$ is chosen as a terminal equality constraint, i.e., $\mathbb{X}_{f}=x_{s}$.

The behavior of the closed EMPC loop is depicted in Figure 3.3. Apparently, despite the terminal constraint, the closed-loop EMPC solution does not converge to the optimal steady state. The explanation of this behavior is simple: the existence of a unique globally optimal steady state does not guarantee optimal operation at steady state. In other words, system (3.10) subject to 3.11 appears to be not optimally operated at steady state. 

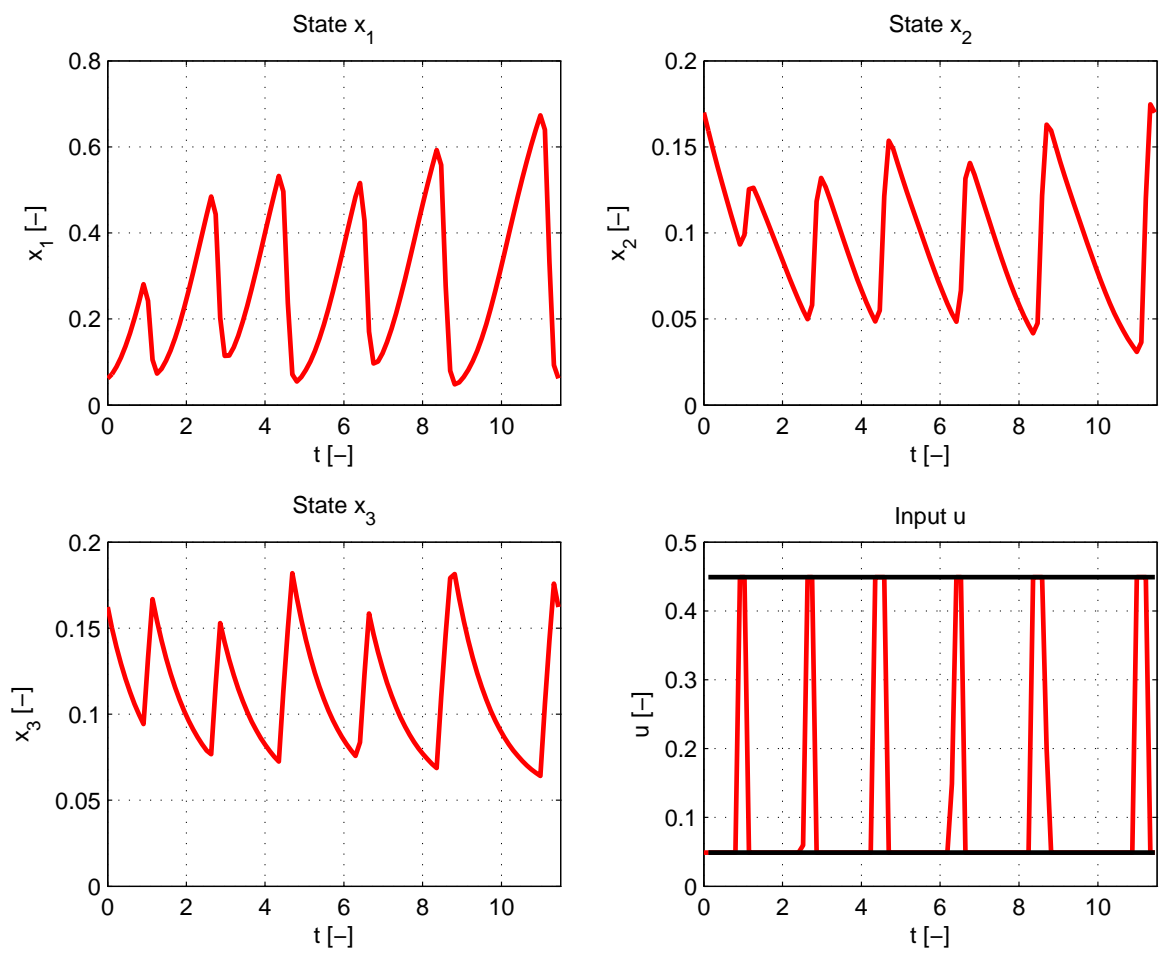

Figure 3.4: Periodic optimal solution to OCP (3.13).

\section{Open-loop Optimal Solution}

In order to clarify this issue, we formulate the following free end-time ocP with periodic boundary conditions:

$$
\min _{u(\cdot), T} \frac{1}{T} \int_{0}^{T}-x_{2}(\tau) \mathrm{d} \tau
$$

subject to $3.10,3.11$ and $x(0)=x(T), \quad T \in[5,20]$.

We solve this OCP using again (Houska, Ferreau, et al., 2011). The solutions are shown in Figure 3.4. We obtain an optimal periodic orbit of length $T^{\star}=11.444$. The average performance along the computed periodic orbit is

$$
\frac{1}{T^{\star}} \int_{0}^{T^{\star}}-x_{2}^{\star}(\tau) \mathrm{d} \tau=-0.09543 .
$$

Recall that the optimal steady state yields an average performance of -0.0846 . Hence, with respect to stage cost $L(x, u)=-x_{2}$, system (3.10) is not optimally operated at steady state and system $(3.10)$ is not strictly dissipative with respect to $L(x, u)=-x_{2}$. 

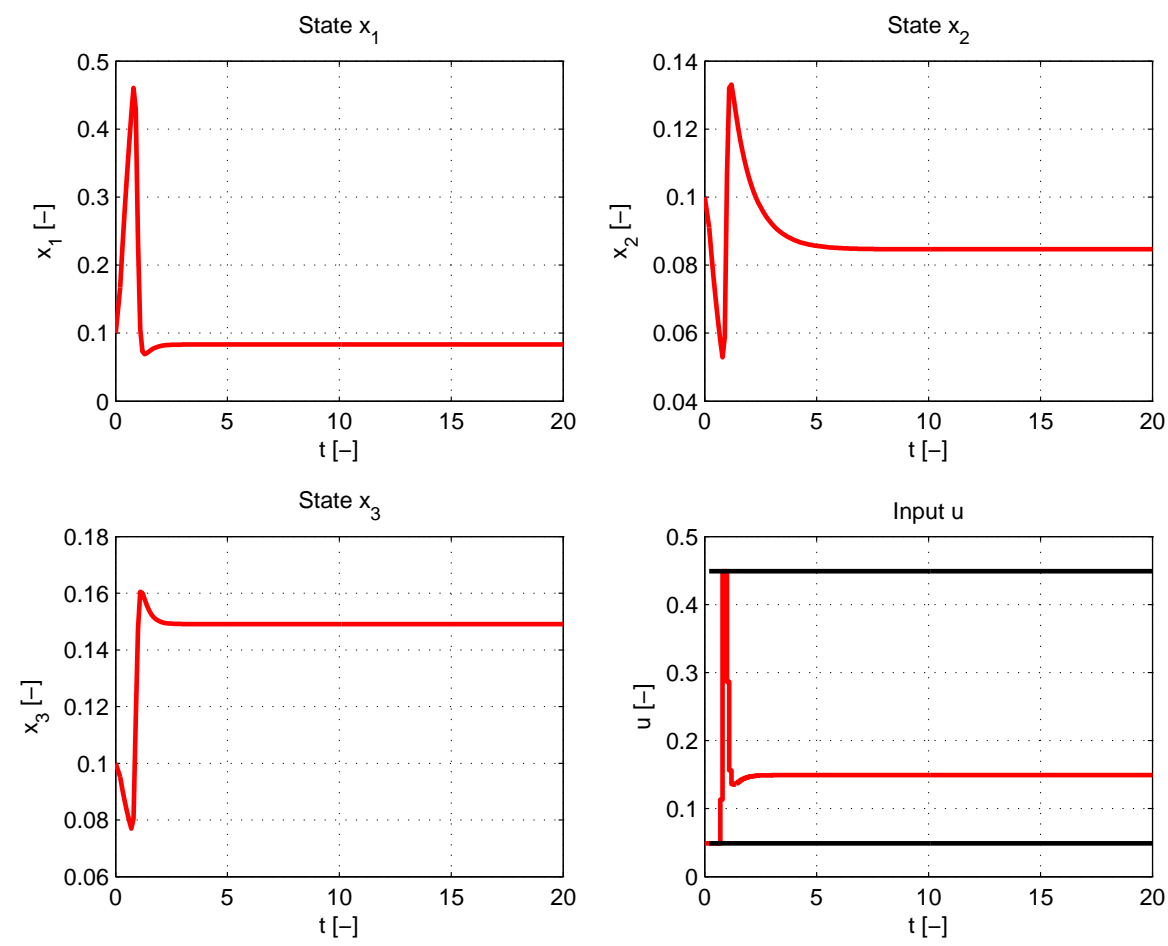

Figure 3.5: Closed-loop EMPC with zero terminal constraint and $L(x, u)=-x_{2}+0.5(u-$ $\left.u_{s}\right)^{2}$.

\section{Closed-loop EMPC with Regularized Objective}

Similar to (Angeli et al., 2012, Diehl et al., 2011) we recover optimal operation at steady state by changing the objective to

$$
L(x, u)=-x_{2}+\omega\left(u-u_{s}\right)^{2}, \quad \omega>0 .
$$

The behavior of the closed EMPC loop for $\omega=0.5$ is depicted in Figure 3.5. As one can see, adding the regularization term $\omega\left(u-u_{s}\right)^{2}$ to the stage cost enforces convergence to the optimal steady state. 


\section{EMPC without Terminal Constraints and Penalties}

In the previous section, we have analyzed how dissipaptivity allows to extend stability results using terminal constraints to more general stage costs. Next, we aim at showing that under suitable assumptions, dissipativity enables the design of EMPC without terminal constraints and penalties. To this end, we will begin by investigating turnpike properties of OCPs and their implications on recursive feasibility.

Hence, for the remainder of this section, we consider NMPC based on the receding horizon solution to the following OCP, which does not include any terminal constraint or penalty:

$$
\begin{array}{rlrl}
V_{N}(x(t)) & :=\min _{u(\cdot \mid t)} \sum_{k=0}^{N-1} \ell(x(k \mid t), u(k \mid t)) & & \\
\text { subject to } & & \\
x(k+1 \mid t) & =f(x(k \mid t), u(k \mid t)), & & k=0, \ldots, N-1 \\
x(0 \mid t) & =x(t) & & \\
(x(k \mid t), u(k \mid t))^{\top} & \in \mathbb{X} \times \mathbb{U}, & &
\end{array}
$$

\subsection{The Turnpike Property}

In this section we introduce and study the turnpike property which will be crucial in our subsequent analysis. In order to ensure that the optimal control problem exhibits this property, we assume the following:

Assumption 4.1 (Strict dissipativity of OCP (4.1)). The exists a bounded non-negative storage function $\lambda: \mathbb{X} \rightarrow \mathbb{R}_{0}^{+}$such that OCP 4.1) is strictly dissipative with respect to $\left(x_{s}, u_{s}\right) \in \operatorname{int}(\mathbb{X} \times \mathbb{U})$ in the sense of Definition 3.2.

Note that throughout this and the following section, we consider OCP (4.1) for initial 
conditions $x_{0} \in \mathbb{X}_{0} \subseteq \mathbb{X}$ in $4.1 \mathrm{c}$.

Assumption 4.2 (Exponential reachability of $x_{s}$ ). For all $x_{0} \in \mathbb{X}_{0}$, there exists an infinite-horizon admissible input $u\left(\cdot ; x_{0}\right), C>0, \rho \in[0,1)$, such that

$$
\left\|\left(x\left(k ; x_{0}, u\left(\cdot ; x_{0}\right)\right), u\left(k ; x_{0}\right)\right)-\left(x_{s}, u_{s}\right)\right\| \leq c \rho^{k}
$$

i.e. the steady state $x_{s}$ is exponentially reachable.

These assumptions allow establishing the following result:

Proposition 4.1 (Turnpike in OCP 4.1)). Let Assumptions 4.1 and 4.2 hold, and suppose that the storage function $\lambda$ is bounded on $\mathbb{X}$. Then, there exists $C<\infty$, such that, for all $x_{0} \in \mathbb{X}_{0}$, we have

$$
\# \mathbb{Q}_{\varepsilon} \geq N-\frac{C}{\alpha_{\ell}(\varepsilon)}
$$

where $\mathbb{Q}_{\varepsilon}:=\left\{k \in\{0, \ldots, N-1\} \mid\left\|\left(x^{\star}\left(k ; x_{0}\right), u^{\star}\left(k ; x_{0}\right)\right)-\left(x_{s}, u_{s}\right)\right\| \leq \varepsilon\right\}$ and $\# \mathbb{Q}_{\varepsilon}$ is the cardinality of $\mathbb{Q}_{\varepsilon}$.

Proof. Let $V_{N}\left(x_{0}\right)$ denote the optimal value function of OCP (4.1); and without loss of generality assume that $\ell\left(x_{s}, u_{s}\right)=0$. The strict dissipation inequality $3.2 \mathrm{~b}$ with bounded storage implies

$$
V_{N}\left(x_{0}\right) \geq
$$

$$
\underbrace{\lambda\left(x^{\star}\left(N, x_{0}\right)\right)-\lambda\left(x_{0}\right)}_{<\bar{\lambda}:=2 \sup _{x \in \mathbb{X}} \lambda(x)<\infty}+\sum_{k=0}^{N-1} \alpha_{\ell}\left(\left\|\left(x^{\star}\left(k ; x_{0}\right), u^{\star}\left(k ; x_{0}\right)\right)-\left(x_{s}, u_{s}\right)\right\|\right) .
$$

Assumption 4.2 gives

$$
V_{N}\left(x_{0}\right) \leq \frac{L_{\ell} c}{1-\rho}
$$

where $L_{\ell}$ is a Lipschitz constant of $\ell$ on $\mathbb{X}$. Noting that $N-\# \mathbb{Q}_{\varepsilon}$ denotes the amount of time an optimal pair $x^{\star}\left(k ; x_{0}\right), u^{\star}\left(k ; x_{0}\right)$ spends outside of an epsilon neighborhood of $x_{s}$, we have

$$
\sum_{k=0}^{N-1} \alpha_{\ell}\left(\left\|\left(x^{\star}\left(k ; x_{0}\right), u^{\star}\left(k ; x_{0}\right)\right)-\left(x_{s}, u_{s}\right)\right\|\right) \geq\left(N-\# \mathbb{Q}_{\varepsilon}\right) \alpha_{\ell}(\varepsilon)
$$


Combining the last three inequalities, we obtain

$$
\# \mathbb{Q}_{\varepsilon} \geq N-\frac{L_{\ell} c(1-\rho)^{-1}-2 \bar{\lambda}}{\alpha_{\ell}(\varepsilon)} .
$$

Hence the assertion follows.

The last result states that strict dissipativity of OCP 4.1) and exponential reachability imply, for all $x_{0} \in \mathbb{X}_{0}$, that the optimal solutions spend most time close to the optimal steady-state pair $\left(x_{s}, u_{s}\right)$. This phenomenon is known as turnpike property of OCPs. The observation that dissipativity implies the existence of a turnpike was first made in (Grüne, 2013), based on a conceptually similar result in (Carlson et al., 1991, Chap. 4). Similar results, replacing the reachability condition of Assumption 4.2 with bounds on the value function $V_{N}$, can be found in (Damm et al., 2014; Grüne, 2013; Grüne and Pannek, 2017). Similar continuous-time results can be found in (Faulwasser et al., 2014, 2017).

Remark 4.1 (Equivalence of turnpike and dissipativity properties). In Section 3 we have commented on the close relation between optimal operation at steady state and dissipativity. As Theorem 4.1 shows under some technical assumptions the implication dissipativity $\Rightarrow$ turnpike, it is fair to ask whether the converse statement $\Leftarrow$ also holds true. We remark without further elaboration that under suitable assumptions this equivalence can be affirmed. The interested reader is referred to (Grüne and Müller, 2016) for discrete-time results and to (Faulwasser et al., 2017) for a continuous-time analysis.

Example 4.1 (Turnpike properties in OCPs). Consider the linear system $x(t+1)=$ $2 x(t)+u(t)$ with $, \mathbb{X}=[-2,2], \mathbb{U}=[-3,3]$ and $x_{0}=2$. Let the ocP be to minimize the stage cost $\ell(x, u)=u^{2}$. The best steady state minimizing the stage cost $l$ is $(0,0)$. Furthermore, it is easy to show that the function $\lambda(x) \equiv 0$ satisfies (3.2a) and on $\mathbb{X}=$ $[-2,2]$ the functions $\lambda_{c}(x)=c-\frac{1}{2} x^{2}, c \geq 2$ satisfy (3.2b). In other words, the system is strictly dissipative on $\mathbb{X}$ with respect to the steady state $\left(x_{s}, u_{s}\right)=(0,0)$.

We consider an increasing sequence of horizons $N=1,3, \ldots, 25$ and solve these OCP $s$. The results are shown in Figure 4.1. As predicted by Proposition 4.1, the optimal solutions show the turnpike property.

Example 4.1 demonstrates that the turnpike property implies a kind of similarity of solutions to an OCP for varying horizons (and varying initial conditions). Put differently, turnpikes are similarity properties of parametric OCPs. 
State $\mathrm{x}$

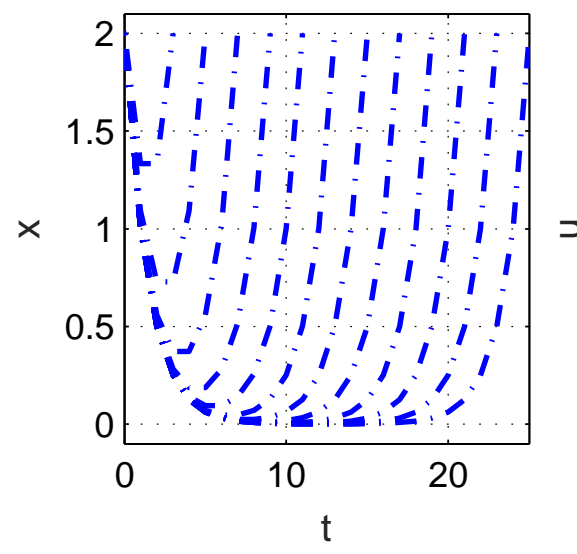

Input u

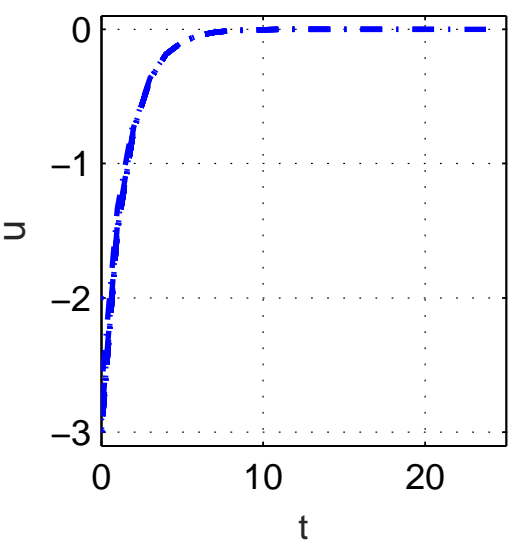

Figure 4.1: Solutions to the OCP from Example 4.1.

\subsection{Recursive Feasibility}

As we will see later, the turnpike plays a crucial role in analyzing EMPC without terminal constraints and penalties. Furthermore, it allows establishing recursive feasibility of oCP (4.1). To this end, we assume the following:

Assumption 4.3 (Local $n_{x}$-step reachability). The Jacobian linearization of system (2.1) at $\left(x_{s}, u_{s}\right)$ is $n_{x}$-step reachable.11

Proposition 4.2 (Recursive feasibility of OCP (4.1)). Let Assumptions 4.144 .3 hold. Then, there exists a finite horizon $N \in \mathbb{N}$ such that, for all $x_{0} \in \mathbb{X}_{0}$, OCP (4.1) is recursively feasible.

Proof. Let $\left(x^{\star}\left(\cdot ; x_{0}\right), u^{\star}\left(\cdot ; x_{0}\right)\right)$ be the optimal pair of ocP 4.1 with initial condition $x_{0}$. Moreover, let $n_{x}$ be the dimension of $x$.

Proposition 4.1 implies that, for all $x_{0} \in \mathbb{X}_{0}$ and any $\varepsilon>0$, we can find a finite horizon $N_{\varepsilon}$ such that there exists $k_{1}$, with $k_{1}+2 n_{x} \leq N$, such that $x^{\star}\left(k_{1} ; x_{0}\right) \in \mathcal{B}_{\varepsilon}\left(x_{s}\right)$. Moreover, Assumption 4.3 i.e. local $n_{x}$-step controllability of (2.1) close to $x_{s}$-implies that on a sufficiently small neighborhood $\mathcal{B}_{\varepsilon}\left(x_{s}\right)$, for all $x_{1}^{\varepsilon}, x_{2}^{\varepsilon} \in \mathcal{B}_{\varepsilon}\left(x_{s}\right)$ there exists admissible controls $u_{1}^{\varepsilon}\left(\cdot ; x_{1}^{\varepsilon}\right)$ and $u_{2}^{\varepsilon}\left(\cdot ; x_{2}^{\varepsilon}\right)$, both defined for $k=0, \ldots, n_{x}-1$, such that

$$
\begin{aligned}
& x\left(n_{x} ; x_{1}^{\varepsilon}, u_{1}^{\varepsilon}\left(\cdot ; x_{1}^{\varepsilon}\right)\right)=x_{s} \quad \text { and } \quad x\left(n_{x} ; x_{s}, u_{2}^{\varepsilon}\left(\cdot ; x_{2}^{\varepsilon}\right)\right)=x_{2}^{\varepsilon} \\
& x\left(k ; x_{1}^{\varepsilon}, u_{1}^{\varepsilon}\left(\cdot ; x_{1}^{\varepsilon}\right)\right) \in \mathbb{X}, x\left(k ; x_{s}, u_{2}^{\varepsilon}\left(\cdot ; x_{2}^{\varepsilon}\right)\right) \in \mathbb{X},
\end{aligned}
$$

\footnotetext{
${ }^{1}$ Recall that local $n$-step reachability of $x^{+}=A x+B u$ implies that starting from $x=0$ one can reach any $x \in \mathbb{R}^{n_{x}}$ within $n_{x}$ time steps; and one can steer any $x \neq 0$ to the origin within $n_{x}$ time steps, cf. (Weiss, 1972). In other words, $n_{x}$-step reachability implies $n_{x}$-step controllability.
} 


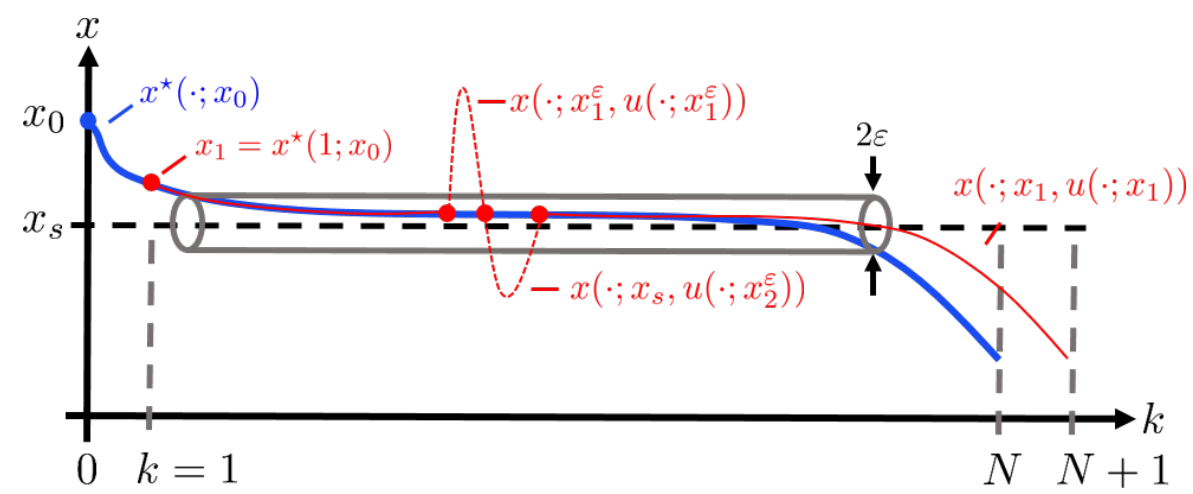

Figure 4.2: Sketch of the trajectory $x\left(\cdot ; x_{1}, u\left(\cdot ; x_{1}\right)\right)$ generated by $u\left(\cdot ; x_{1}\right)$ from 4.3$)$.

whereby the constraint satisfaction in $4.2 \mathrm{~b}$ holds for $k=0, \ldots, n_{x}$.

In order to construct $u_{1}^{\varepsilon}\left(\cdot ; x_{1}^{\varepsilon}\right)$ and $u_{2}^{\varepsilon}\left(\cdot ; x_{2}^{\varepsilon}\right)$ one may rely on methods sketched in (Weiss, 1972). Recall that $u^{\star}\left(\cdot ; x_{0}\right)$ denotes the optimal input for OCP 4.1) given the initial condition $x_{0} \in \mathbb{X}_{0}$. Let $x_{1}=f\left(x_{0}, u^{\star}\left(0 ; x_{0}\right)\right)$ and consider the following input

$$
u\left(k ; x_{1}\right)=\left\{\begin{array}{ll}
u^{\star}\left(k+1 ; x_{0}\right) & k=0, \ldots, k_{1}-2 \\
u_{1}^{\varepsilon}\left(k ; x_{1}^{\varepsilon}\right) & k=k_{1}-1, \ldots, k_{1}-1+n_{x} \\
u_{2}^{\varepsilon}\left(k ; x_{2}^{\varepsilon}\right) & k=k_{1}+n_{x}, \ldots, k_{1}-1+2 n_{x} \\
u^{\star}\left(k ; x_{0}\right) & k=k_{1}+2 n_{x}, \ldots, N-1
\end{array} .\right.
$$

As we may choose the horizon $N$, we can pick it such that $x^{\star}\left(k_{1}-1+2 n_{x} ; x_{0}\right) \in \mathcal{B}_{\varepsilon}\left(x_{s}\right)$. Now, in the construction of $u\left(\cdot ; x_{1}\right)$, choose $x_{2}^{\varepsilon}:=x^{\star}\left(k_{1}+2 n_{x} ; x_{0}\right)$. In other words, the sequence of inputs $u_{1}^{\varepsilon}\left(\cdot ; x_{1}^{\varepsilon}\right)$ and $u_{2}^{\varepsilon}\left(\cdot ; x_{2}^{\varepsilon}\right)$ connects $x^{\star}\left(k_{1} ; x_{0}\right)$ and $x^{\star}\left(k_{1}+2 n_{x} ; x_{0}\right)$ in an admissible way.

The trajectory generated by $u\left(\cdot ; x_{1}\right)$ is sketched in Figure 4.2 . It is readily seen that (4.2) implies feasibility of $u\left(\cdot ; x_{1}\right)$ from 4.3) in OCP 4.1) with initial condition $x_{1}$.

The observation that the turnpike property from Proposition 4.1 can be used to establish recursive feasibility for sufficiently long horizon has been first made in a continuoustime setting in (Faulwasser and Bonvin, 2015a b).

Remark 4.2 (Recursive feasibility with local stabilizability).

Observe that, without significant technical difficulties, Assumption 4.3 can be relaxed to local linear stabilizability of system (2.1). To this end, one swaps $u_{1}^{\varepsilon}\left(\cdot ; x_{1}^{\varepsilon}\right)$ and $u_{2}^{\varepsilon}\left(\cdot ; x_{2}^{\varepsilon}\right)$ in (4.3) with a locally stabilizing feedback, which is to be considered for all $k=k_{1}, \ldots, N-1$. Picking a horizon $N$ long enough such that at time $k_{1}$ the optimal solution $x^{\star}\left(k_{1} ; x_{0}\right)$ is inside a suitable level set of a local Lyapunov function corresponding to the locally stabi- 
lizing feedback shows recursive feasibility. Here, in order to simplify the later derivations, we rely on the construction 4.3).

\subsection{Practical Stability}

Before stating the main stability result of this section, we recall the following definition given in (Grüne and Stieler, 2014a):

Definition 4.1 (Local practical asymptotic stability). Consider the closed-loop system (2.6), generated by the feedback $\mu$. The point $x_{s}$ is said to be a locally practically asymptotically stable w.r.t. $\rho>0$ on a set $\mathbb{S} \subseteq \mathbb{X}$, if there exists $\beta \in \mathcal{K} \mathcal{L}$ such that

$$
\left\|x\left(k ; x_{0}, \mu_{N}(x)\right)-x_{s}\right\| \leq \max \left\{\beta\left(\left\|x\left(k ; x_{0}, \mu_{N}(x)\right)-x_{s}\right\|, k\right), \rho\right\}
$$

holds for all $k \in \mathbb{N}_{0}$ and all $x_{0} \in \mathbb{S}$.

The next technical result is quite standard. It gives sufficient conditions for local practical stability.

Proposition 4.3 (Lyapunov function for local practical asymptotic stability). Consider the closed-loop system (2.6) generated by the feedback $\mu$, and, for some finite $\delta_{2}, \delta_{3} \in \mathbb{R}^{+}$, let $V: \mathbb{X} \rightarrow \mathbb{R}$ satisfy

$$
\begin{array}{ll}
\alpha_{1}\left(\left\|x-x_{s}\right\|\right) \leq V(x) \leq \alpha_{2}\left(\left\|x-x_{s}\right\|\right)+\delta_{2}, & \forall x \in \mathbb{X} \\
V\left(f(x, \mu(x)) \leq V(x)-\alpha_{3}\left(\left\|x-x_{s}\right\|\right)+\delta_{3},\right. & \forall x \in \mathbb{S}(L) .
\end{array}
$$

Suppose either $\mathbb{S}(L)=\mathbb{X}$ or $\mathbb{S}(L)=\{x \in \mathbb{X} \mid V(x) \leq L\}$, with $\mathbb{X}$ compact and

$$
L \geq \alpha_{2}\left(\alpha_{3}^{-1}\left(\delta_{3}\right)+\delta_{3}\right)+\delta_{2}+\delta_{3}
$$

Then, there exists $\beta \in \mathcal{K} \mathcal{L}$ such that, for all $x_{0} \in \mathbb{S}(L)$,

$$
\left\|x\left(k ; x_{0}, \mu(x)\right)-x_{s}\right\| \leq \max \left\{\beta\left(\left\|x\left(k ; x_{0}, \mu(x)\right)-x_{s}\right\|, k\right), \rho\right\}
$$

with $\rho=\alpha_{1}^{-1}\left(\alpha_{2}\left(\alpha_{3}^{-1}\left(\delta_{3}\right)+\delta_{3}\right)+\delta_{2}+\delta_{3}\right)$.

Proof. The proof is an adaptation of results presented in (Grüne and Pannek, 2017, Chap. 2) and (Grüne and Stieler, 2014a). The crucial difference to the results therein is the constant $\delta_{2}$ in $4.5 \mathrm{a}$. 
Step 1: Consider $L_{1}>\alpha_{2}\left(\alpha_{3}^{-1}\left(\delta_{3}\right)+\delta_{3}\right)+\delta_{2}+\delta_{3}$. For all $x \in \mathbb{S}\left(L_{1}\right)$, if $\left\|x-x_{s}\right\|>$ $\alpha_{3}^{-1}\left(\delta_{3}\right)$, then

$$
V\left(f(x, \mu(x)) \leq V(x)-\alpha_{3}\left(\left\|x-x_{s}\right\|\right)+\delta_{3} \leq V(x) \leq L_{1} .\right.
$$

Moreover, if $\left\|x-x_{s}\right\|<\alpha_{3}^{-1}\left(\delta_{3}\right)+\delta_{3}$, then $V(x)<\alpha_{2}\left(\alpha_{3}^{-1}\left(\delta_{3}\right)+\delta_{3}\right)+\delta_{2}$. Inequality 4.5b) gives

$$
V\left(f(x, \mu(x)) \leq \alpha_{2}\left(\alpha_{3}^{-1}\left(\delta_{3}\right)+\delta_{3}\right)+\delta_{2}+\delta_{3}<L_{1} .\right.
$$

Hence the set $\mathbb{S}\left(L_{1}\right)$ is forward invariant.

Step 2: Consider $L_{2}=\alpha_{2}\left(\alpha_{3}^{-1}\left(\delta_{3}\right)+\delta_{3}\right)+\delta_{2}+\delta_{3}$. If $\left\|x-x_{s}\right\|>\alpha_{3}^{-1}\left(\delta_{3}\right)$, then the same reasoning as above shows

$$
V\left(f(x, \mu(x)) \leq V(x) \leq L_{2}\right.
$$

If $\left\|x-x_{s}\right\| \leq \alpha_{3}^{-1}\left(\delta_{3}\right)$, then $V(x) \leq \alpha_{2}\left(\alpha_{3}^{-1}\left(\delta_{3}\right)+\delta_{3}\right)+\delta_{2}$. This gives

$$
V\left(f(x, \mu(x)) \leq V(x)-\alpha_{3}\left(\left\|x-x_{s}\right\|\right)+\delta_{3}\right.
$$

$$
\leq \alpha_{2}\left(\alpha_{3}^{-1}\left(\delta_{3}\right)+\delta_{3}\right)+\delta_{2}+\delta_{3}=L_{2}
$$

Hence, the set $\mathbb{S}\left(L_{2}\right)$ is forward invariant.

Step 3: W.l.o.g. pick $c>1$ such that $\alpha_{2}\left(\alpha_{3}^{-1}\left(c \delta_{3}\right)\right)+\delta_{2} \leq L_{2}$. For $x \in \mathbb{S}\left(L_{1}\right) \backslash \mathbb{S}\left(L_{2}\right)$, we have $V(x)>L_{2}$ and thus $\alpha_{3}\left(\left\|x-x_{s}\right\|\right) \geq \alpha_{3}\left(\alpha_{2}^{-1}\left(L_{2}-\delta_{2}\right)\right) \geq c \delta_{3}$. Thus, for all $x \in \mathbb{S}\left(L_{1}\right) \backslash \mathbb{S}\left(L_{2}\right)$, we have $\alpha_{3}\left(\left\|x-x_{s}\right\|\right)-\delta_{3} \geq\left(1-\frac{1}{c}\right) \alpha_{3}\left(\left\|x-x_{s}\right\|\right)$ and

$$
V\left(f(x, \mu(x)) \leq V(x)-\left(1-\frac{1}{c}\right) \alpha_{3}\left(\left\|x-x_{s}\right\|\right)\right.
$$

Step 4: Now, observe that for all $x \in \mathbb{S}\left(L_{1}\right) \backslash \mathbb{S}\left(L_{2}\right)$, we have $\left\|x-x_{s}\right\| \geq \alpha_{3}\left(\delta_{3}\right)$. Consider

$$
c_{1}:=\sup _{x \in \mathbb{S}\left(L_{1}\right) \backslash \mathbb{S}\left(L_{2}\right)} \alpha_{2}\left(\left\|x-x_{s}\right\|\right)-\delta_{2}
$$

which gives

$$
\tilde{\alpha}_{2}\left(\left\|x-x_{s}\right\|\right):=\frac{c_{1}}{\alpha_{3}\left(\delta_{3}\right)}\left\|x-x_{s}\right\| \geq \alpha_{2}\left(\left\|x-x_{s}\right\|\right)-\delta_{2}, \quad \forall x \in \mathbb{S}\left(L_{1}\right) \backslash \mathbb{S}\left(L_{2}\right) .
$$

Finally, with straightforward modifications to the proof of (Grüne and Pannek, 2017, Thm. 2.19 and Thm. 2.20) one establishes the bound in Equation (4.7). 
In order to prove Theorem 3.2 in Section 3.2 we used that in the presence of suitable terminal constraints, rotating the stage cost and terminal penalty by the storage function $\lambda$ does not change the optimal solutions, cf. Lemma 3.2. For ocP (4.1), however, the situation is slightly different as rotating the stage cost yields

$$
\sum_{k=0}^{N-1} \tilde{\ell}(x(k \mid t), u(k \mid t))=\lambda(x)-\lambda(x(N \mid t))+\sum_{k=0}^{N-1} \ell(x(k \mid t), u(k \mid t)) .
$$

Observe that the value of $\lambda(x(N \mid t))$ will depend on the chosen input sequence. In other words, pure rotation of the objective without introducing an additional terminal constraint alters optimal solutions. Subsequently, we will have to take this into account and thus we will proceed in two steps: First we show that using an appropriate value function, we can establish convergence to a neighborhood of the optimal steady state. Second we use the value function of a modified, i.e. rotated version, of ocP (4.1) to show that with increasing horizon this neighborhood can be made arbitrarily small.

Theorem 4.1 (Practical stability of EMPC without terminal constraints). Let Assumptions 4.14 .3 hold and suppose that $\mathbb{X}$ is compact. Then, there exist a sufficiently large horizon $N \in \mathbb{N}$, such that the closed-loop system (2.6) arising from the receding horizon solution to OCP (4.1) has the following properties:

(i) If, for the horizon $N \in \mathbb{N}$, OCP 4.1) is feasible for $t=0$ and $x(0) \in \mathbb{X}_{0}$, then it is feasible for all $k \in \mathbb{N}$.

(ii) For all $x(0) \in \mathbb{X}_{0}$, there exist $\rho \in \mathbb{R}^{+}$and $\beta \in \mathcal{K} \mathcal{L}$ such that the closed-loop trajectories generated by (2.6) satisfy

$$
\left\|x(t)-x_{s}\right\| \leq \max \left\{\beta\left(\left\|x(t)-x_{s}\right\|, t\right), \rho\right\} .
$$

Proof. Part (i) directly follows from Proposition 4.2. In order to prove Part (ii), we consider the following shifted value function

$$
\hat{V}_{N}(x)=\lambda(x)+V_{N}(x)
$$

with $V_{N}$ from 4.1a and $\lambda$ being a bounded non-negative storage function of ocP 4.1 satisfying (3.2b) along any optimal solution. Note that this shift of the value function is done without loss of generality, as shifting the objective in OCP 4.1 by a constant depending only on the initial condition $x$ does not alter the optimal solutions. 
Similar to the proof of Theorem 2.1, we consider

$$
\hat{V}_{N}(x(t+1))-\hat{V}_{N}(x(t)) \leq \lambda(x(t+1))+J_{N}(x(t+1), u(\cdot \mid t+1))-\hat{V}_{N}(x(t)),
$$

where $J_{N}$ is the performance generated by the input $u(\cdot \mid t+1)$ defined in (4.3) and $x(\cdot \mid t+1)$ denotes the corresponding trajectory. For sake of simplified notation, we define the right hand side of the last inequality as

$$
\Delta(t):=\lambda(x(t+1))+J_{N}(x(t+1), u(\cdot \mid t+1))-\hat{V}_{N}(x(t)),
$$

and rewrite it as follows

$$
\begin{aligned}
\Delta(t) & =\lambda(x(t+1))-\lambda(x(t))-\ell\left(x(t), u^{\star}(0 \mid t)\right) \\
& +\sum_{k=0}^{k_{1}-1} \ell(x(k \mid t+1), u(k \mid t+1))-\sum_{k=1}^{k_{1}} \ell\left(x^{\star}(k \mid t), u^{\star}(k \mid t)\right) \\
& +\sum_{k=k_{1}}^{k_{1}-1+2 n_{x}} \ell(x(k \mid t+1), u(k \mid t+1))-\sum_{k=k_{1}+1}^{k_{1}-1+2 n_{x}} \ell\left(x^{\star}(k \mid t), u^{\star}(k \mid t)\right) \\
& +\sum_{k=k_{1}+2 n_{x}}^{N-1} \ell(x(k \mid t+1), u(k \mid t+1))-\sum_{k=k_{1}+2 n_{x}}^{N-1} \ell\left(x^{\star}(k \mid t), u^{\star}(k \mid t)\right) .
\end{aligned}
$$

By construction of $u(\cdot \mid t+1)$ in 4.3 and as we consider the nominal case -i.e. no plantmodel mismatch - the sums in the second and fourth line of the last equation cancel each other.

Now, assume without loss of generality that $\ell\left(x_{s}, u_{s}\right)=0$ and let $L_{\ell}$ be a Lipschitz constant of $\ell$ on $\mathbb{X} \times \mathbb{U}$. Then

$$
\begin{gathered}
\sum_{k=k_{1}}^{k_{1}-1+2 n_{x}} \ell(x(k \mid t+1), u(k \mid t+1))-\sum_{k=k_{1}+1}^{k_{1}-1+2 n_{x}} \ell\left(x^{\star}(k \mid t), u^{\star}(k \mid t)\right) \leq \\
\ell\left(x\left(k_{1} \mid t+1\right), u\left(k_{1} \mid t+1\right)\right)+2 n_{x} L_{\ell} c(\varepsilon) \leq\left(2 n_{x}+1\right) L_{\ell} c(\varepsilon)
\end{gathered}
$$

holds, whereby due to the turnpike property from Proposition 4.1 and due to local controllability close to $x_{s}$, the constant $c(\varepsilon)$ depends on $N$. Invoking that the strict dissipation inequality $3.2 \mathrm{~b}$ ) holds along optimal solutions of ocP (4.1), we obtain

$$
\hat{V}_{N}(x(t+1))-\hat{V}_{N}(x(t)) \leq \Delta(t) \leq-\alpha_{\ell}\left(\left\|x(t)-x_{s}\right\|\right)+\left(2 n_{x}+1\right) L_{\ell} c(\varepsilon) .
$$


Furthermore, strict dissipativity with non-negative bounded storage implies the following lower bound

$$
\hat{V}_{N}(x) \geq \lambda(x)-\lambda\left(x^{\star}(N \mid t)\right)+\sum_{k=0}^{N-1} \ell\left(x^{\star}(k \mid t), u^{\star}(k \mid t)\right) \geq \alpha_{\ell}\left(\left\|x-x_{s}\right\|\right)
$$

for all $x \in \mathbb{X}_{0}$. Moreover, exponential reachability (Assumption 4.2 gives, for all $x \in \mathbb{X}_{0}$,

$$
\hat{V}_{N}(x) \leq L_{\ell}\left\|x-x_{s}\right\|+(N-1) \bar{\ell}
$$

with $\bar{\ell}=\sup _{(x, u) \in \mathbb{X} \times \mathbb{U}} \ell(x, u)$. Applying Proposition 4.3 finishes the proof.

So far, we have shown that EMPC without terminal constraints leads to practical asymptotic stability. However, the proof above does not provide insight in the relation between $\rho$, which is the size of the neighborhood the closed-loop solutions converge to, and the prediction horizon $N$. Ideally, we would like to replace $\rho$ by a function $\rho(N)$ which tends to 0 as $N$ tends to $\infty$. To this end, we need to replace the terms $\left(2 n_{x}+1\right) L_{\ell} c(\varepsilon)$ and $(N-1) \bar{\ell}$ in $4.10 \mathrm{a}$ and $4.10 \mathrm{c}$, respectively, by terms which converge to 0 as $N \rightarrow \infty$. The next lemma will provide an important auxiliary result for this purpose. Similar to Section 3.2 we denote by $\tilde{V}_{N}$ the corresponding value function of OCP (4.1) using the rotated stage cost $\tilde{\ell}$ instead of $\ell$.

Lemma 4.1 (Relation between $\tilde{V}_{N}$ and $V_{N}$ ). Let Assumptions 4.1 4.3 hold. Moreover,

1. let there exist $\gamma_{V} \in \mathcal{K}$ such that for each $N \in \mathbb{N}$ and all $x \in \mathbb{X}_{0}\left|\tilde{V}_{N}(x)-\tilde{V}_{N}\left(x_{s}\right)\right| \leq$ $\gamma_{\tilde{V}}\left(\left\|x-x_{s}\right\|\right)$,

2. and let the storage function $\lambda$ be continuous at $x=x_{s}$.

Then

$$
\tilde{V}_{N}(x)=V_{N}(x)+\lambda(x)-V_{N}\left(x_{s}\right)+R(x, N)
$$

with $|R(x, N)| \leq \nu\left(\left\|x-x_{s}\right\|\right)+\omega(N), \nu \in \mathcal{K}, \omega \in \mathcal{L}$.

The proof of this result uses the dynamic programming principle. It can be found in (Grüne and Pannek, 2017, Lem. 8.31). Based on this, one can show the following relation between $\rho$ in Part (ii) of Theorem 4.1 and the prediction horizon $N$.

Theorem 4.1. Under the Assumptions of Theorem 4.1 and Lemma 4.1 the assertions of Theorem 4.1 hold with $\rho=\rho(N)$ where $\rho(N) \rightarrow 0$ for $N \rightarrow \infty$. 
The proof of this result follows from Proposition 4.3 if we replace $\left(2 n_{x}+1\right) L_{\ell} c(\varepsilon)$ and $(N-1) \bar{\ell}$ in (4.10a) and 4.10c), respectively, by terms which converge to 0 as $N \rightarrow \infty$. For 4.10a), this can be done because for $x \approx x_{s}$ from controllability of the linearization one can conclude that the controls $u_{1}^{\varepsilon}\left(\cdot \mid x_{1}^{\varepsilon}\right)$ and $u_{2}^{\varepsilon}\left(\cdot \mid x_{2}^{\varepsilon}\right)$ from 4.2 can be chosen to have values close to $u_{s}$. Since strict dissipativity implies that the optimal trajectory also stays close to $x_{s}$, see (Grüne, 2013, Lem. 6.3), the values of the two trajectories are close to each other, and thus we can construct the improved bound in 4.10a).

For (4.10c), we modify the definition of $\hat{V}_{N}$ from 4.8 to $\hat{V}_{N}(x)=\lambda(x)+V_{N}(x)-$ $V_{N}\left(x_{s}\right)$, noting that this does not affect the value of the increment $\hat{V}_{N}(x(t+1))-\hat{V}_{N}(x(t))$. Then (4.11) together with Assumption 1. of Lemma 4.1 can be used in order to replace $(N-1) \ell$ in 4.10 c by a term vanishing as $N \rightarrow \infty$. For a detailed proof we refer to (Grüne and Stieler, 2014b, Thm. 3.7) or (Grüne and Pannek, 2017, Thm. 8.33).

Remark 4.3 (Extension to continuous-time systems). The results of Theorem 4.1 and Theorem 4.1 have been extended to the continuous-time setting. The main difference to the derivations above is that in continuous-time settings one does not need the additional arguments from the proof of Theorem 4.1. Instead, one shows that the size of the neighborhood shrinks with increasing the prediction horizon and decreasing the sampling time, see (Faulwasser and Bonvin, 2015b).

Moreover, whenever the turnpike is exact-i.e. for sufficiently long horizons the optimal solutions enter the steady state exactly at some point and might leave towards the end of the horizon-one can show that EMPC without terminal constraints enforces finitetime convergence, see (Faulwasser and Bonvin, 2015b, 2017). Furthermore, it shown in (Faulwasser and Bonvin, 201\%, Prop. 1) that in case of exact turnpikes, EMPC with finite horizon recovers infinite-horizon optimal performance. However, it is not fully understood yet, under which conditions turnpikes are exact or not.

The important fact shown by Theorem 4.1 is that, even without terminal constraints or penalties, by increasing the prediction horizon $N$ one can enforce that the closedloop system (2.6) arising from the receding horizon solution to OCP 4.1 converges asymptotically into any arbitrarily small neighborhood of the optimal steady state. Put differently, provided strict dissipativity and exponential reachability hold, for sufficiently long horizons EMPC without terminal constraints finds the optimal steady state without any need for explicit computation of the optimal steady state. Furthermore, we have seen that the turnpike property implies recursive feasibility. As it is straightforward to show that also in case of stabilizing NMPC the turnpike property (without the typical leaving arc) can be observed, we note without further elaboration that the recursive 

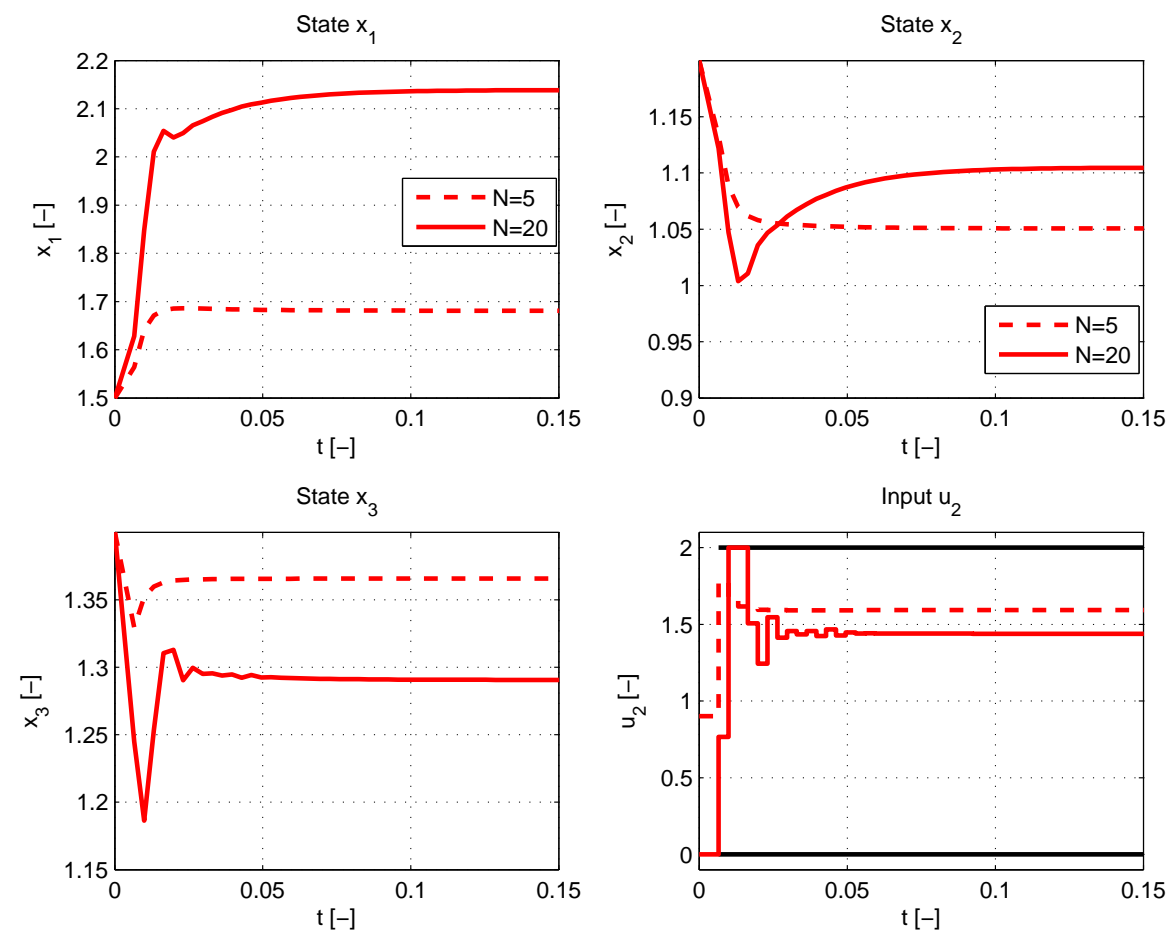

Figure 4.3: EMPC closed loop solution for the Van de Vusse reactor without terminal constraints, $N=5$ (dashed) and $N=20$ (solid).

feasibility properties of Proposition 4.2 also hold for stabilizing NMPC without terminal constraints.

\subsection{Example - Chemical Reactor with Dissipativity}

We revisit the Van de Vusse reactor from Section 3.3. Without terminal constraints, the solutions do not converge to $x_{s}$. Moreover, the limit equilibrium depends on the optimization horizon $N$, as it is expected from the merely practical stability of the closed loop system. Figure 4.3 illustrates this fact, showing the solutions for horizons $N=5$ (dashed) and $N=20$ (solid).

In these simulations the closed loop trajectories converge to an equilibrium $x_{\infty}:=$ $\lim _{t \rightarrow \infty} x(t)$. Theorem 4.1 shows that the distance of this equlibrium $x_{\infty}$ to $x_{s}$ should tend to 0 as $N$ increaes. Figure 4.4 shows that this is indeed the case and that the convergence is even exponentially fast. Note that this exponential convergence can be rigorously established for certain classes of systems, see (Grüne and Stieler, 2014b, Thm 3.7). For determining $x_{\infty}$ for this figure, the closed loop solution was computed until the first 10 significant digits of $L\left(x(t), \mu_{N}(x(t))\right)$ had stabilized. 


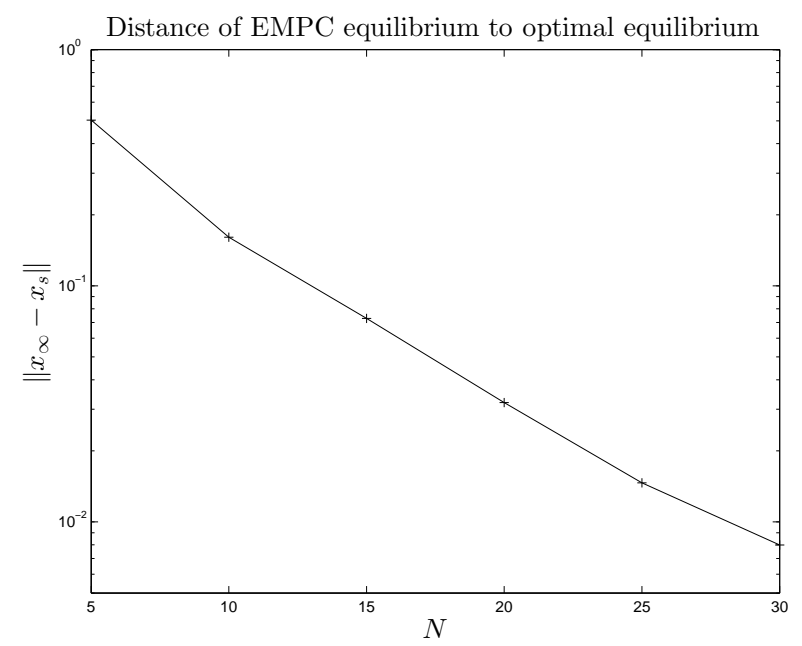

Figure 4.4: Distance $\left\|x_{\infty}-x_{s}\right\|_{2}$ without terminal constraints for $N=5, \ldots, 30$. 



\section{Performance Bounds}

In EMPC, the optimal control problem to be solved in closed loop is not merely an auxiliary problem with the purpose of stabilizing a pre-defined setpoint. Rather, it encodes economic quantities to be optimized, like low energy consumption, high yield or similarly. In order to be economically efficient, one thus needs that the closed loop inherits some of the optimality properties of the open loop predictions computed in each MPC step.

This is indeed the case and in this section we will summarize some of the results available for estimating the closed loop performance of an economic MPC scheme. There are different types of performance measures one can consider for this purpose.

The first is the infinite horizon averaged performance given by

$$
\bar{J}_{\infty}^{c l}\left(x_{0}, \mu_{N}\right):=\limsup _{T \rightarrow \infty} \frac{1}{T} \sum_{t=0}^{T-1} \ell\left(x(t), \mu_{N}(x(t))\right),
$$

where $x(t)$ solves 2.6 .

The second criterion is the infinite horizon non-averaged performance given by

$$
J_{\infty}^{c l}\left(x_{0}, \mu_{N}\right):=\limsup _{T \rightarrow \infty} \sum_{t=0}^{T-1} \ell\left(x(t), \mu_{N}(x(t))\right),
$$

where $x(t)$ again solves (2.6). However, even if $\left|V_{\infty}\left(x_{0}\right)\right|<\infty$, it is not guaranteed that $J_{\infty}^{c l}\left(x_{0}, \mu_{N}\right)$ assumes a finite value, because the inability to converge to the optimal equilibrium exactly causes small deviations from the optimal trajectory in each step of the EMPC scheme, which may add up to an infinite error. It is therefore necessary to also look at the finite horizon non-averaged performance which for each $T \in \mathbb{N}$ is given by

$$
J_{T}^{c l}\left(x_{0}, \mu_{N}\right):=\sum_{t=0}^{T-1} \ell\left(x(t), \mu_{N}(x(t))\right) .
$$

We note that this expression is also meaningful if $\left|V_{\infty}\left(x_{0}\right)\right|=\infty$, while the consideration of $J_{\infty}^{c l}$ only makes sense if the infinite horizon optimal control problem is well defined. 
The standing assumptions we impose throughout the rest of this section are the following:

- We assume that the optimal control problem is strictly dissipative in the sense of Definition 3.1.

- In case terminal conditions are used we impose the condition from Assumption 2.2 on the rotated cost.

- Moreover, we assume continuity at $x=x_{s}$ of the optimal value functions $V_{N}$ for the original and the rotated cost from 3.6a), uniformly in $N$ (as in Assumption 1. of Lemma 4.1), as well as of $V_{\infty}$ and of the storage function $\lambda$ from Definition 3.1 .

Proofs for all subsequent statements which work under these conditions can be found in (Grüne and Pannek, 2017, Chap. 8). Below, we cite those references which contain the earliest version of the respective results we are aware of. These may occcasionally use slightly different assumptions.

\subsection{Averaged Performance}

We start by stating infinite horizon averaged performance results. For problems with terminal constraints, (Amrit et al., 2011, Thm. 18) implies the identity

$$
\bar{J}_{\infty}^{c l}\left(x_{0}, \mu_{N}\right) \leq \ell\left(x_{s}, u_{s}\right)
$$

We note that this inequality holds without any dissipativity assumption. Under our standing assumptions in this section, which include dissipativity, Theorem 3.1 implies optimal operation at the steady state $x_{s}$. Hence, $\ell\left(x_{s}, u_{s}\right)$ is the best possible value for $\bar{J}_{\infty}^{c l}\left(x_{0}, \mu_{N}\right)$, i.e., we obtain optimal infinite horizon averaged performance. This is actually not completely surprising, because the stability result from Theorem 3.2 implies $x(t) \rightarrow x_{s}$ and $\mu_{N}(x(t)) \rightarrow u_{s}$ as $t \rightarrow \infty$ from which the estimate for $\bar{J}_{\infty}^{c l}\left(x_{0}, \mu_{N}\right)$ follows quite straightforwardly.

For problems without terminal constraints we cannot in general expect that the closedloop solution converges to $x_{s}$. Hence, we have to expect an additional error term. Consequently, (Grüne, 2013, Thm. 4.2), used here with $\ell_{0}=\ell\left(x_{s}, u_{s}\right)$ establishes the estimate

$$
\bar{J}_{\infty}^{c l}\left(x_{0}, \mu_{N}\right) \leq \ell\left(x_{s}, u_{s}\right)+\delta(N)
$$


with $\delta \in \mathcal{L}$, i.e., we get approximately optimal infinite horizon averaged performance with the error term $\delta(N)$ which tends to 0 as $N \rightarrow \infty$.

\subsection{Non-averaged Performance}

The infinite horizon averaged performance is a useful criterion when considering very long time horizons. However, it does not say anything about the performance on finite horizons. Indeed, on a finite time horizon trajectories could produce arbitrary large costs and still satisfy $\bar{J}_{\infty}^{c l}\left(x_{0}, \mu_{N}\right) \leq \ell\left(x_{s}, u_{s}\right)$. Hence, we need non averaged estimates in order to exclude this. Assuming $\left|V_{\infty}\left(x_{0}\right)\right|<\infty, 1$ it makes sense to look at the infinite horizon non-averaged performance. With terminal constraints, in Theorem 5.1 of (Grüne and Panin, 2015) it was proved that

$$
J_{\infty}^{c l}\left(x_{0}, \mu_{N}\right) \leq V_{\infty}\left(x_{0}\right)+\delta(N)
$$

with $\delta \in \mathcal{L}$. Hence, we obtain approximately infinite horizon non-averaged optimality with an error term $\delta(N)$ which tends to 0 as $N \rightarrow \infty$.

For problems without terminal constraints such a strong result cannot be expected. This is because $x(t)$ may not converge to $x_{s}$, hence $\ell\left(x(t), \mu_{N}(x(t))\right) \rightarrow 0$ cannot be expected and consequently $J_{\infty}^{c l}\left(x_{0}, \mu_{N}\right)$ will in general not attain a finite value. A remedy for this problem is to consider only a finite piece $x(0), \ldots, x(T)$ of the MPC closed loop and extend this by an infinite horizon optimal trajectory starting in $x(T)$. Proceeding this way leads to the estimate

$$
J_{T}^{c l}\left(x_{0}, \mu_{N}\right)+V_{\infty}(x(T)) \leq V_{\infty}\left(x_{0}\right)+T \hat{\delta}(N)
$$

with $\hat{\delta} \in \mathcal{L}$, first proved in (Grüne, 2016. Thm. 4.4). Hence, each initial piece of length $T$ of the MPC closed loop is the initial piece of an approximately infinite horizon trajectory with error $T \delta(N)$, where $\delta(N) \rightarrow 0$ as $N \rightarrow \infty$.

\footnotetext{
${ }^{1}$ Note that this implies $\ell\left(x_{s}, u_{s}\right)=0$, which can, however, always been achieved by adding an appropriate constant to $\ell$, which does not change the finite horizon optimal trajectories. A way to avoid the assumption $\left|V_{\infty}\left(x_{0}\right)\right|<\infty$ is to use the concept of overtaking optimality, see (Carlson et al., 1991). The use of this concept in an EMPC context is currently under investigation.
} 


\subsection{Transient Performance}

In case the infinite horizon optimal control problem is not well posed (i.e., if $\left|V_{\infty}\left(x_{0}\right)\right|=$ $\infty)$, it is still possible to define a meaningful non-averaged performance result. The reason for this lies in the observation that under our standing assumptions in this section the closed loop for the terminal constrained problem is asymptotically stable, i.e., according to Theorem 3.2 it satisfies

$$
\left\|x(t)-x_{s}\right\| \leq \beta\left(\left\|x_{0}-x_{s}\right\|, t\right)
$$

for a function $\beta \in \mathcal{K} L$. For the problem without terminal constraints, Theorem 4.1 still implies practical asymptotic stability, i.e., the existence of $\beta \in \mathcal{K} L$ and $\rho \in \mathcal{L}$ such that

$$
\left\|x(t)-x_{s}\right\| \leq \max \left\{\beta\left(\left\|x_{0}-x_{s}\right\|, t\right), \rho(N)\right\}
$$

holds.

Hence, in both cases, during the transient phase the closed loop trajectory will run from the initial condition to a small neighbourhood of the optimal steady state $x_{s}$. The concept of transient optimality now formalizes that (at least approximately) the closed loop trajectory is the cheapest among all trajectories running from the initial state to this neighbourhood. In order to state this mathematically, we define

$$
\mathbb{U}_{\kappa}^{T}\left(x_{0}\right):=\left\{u \in \mathbb{U}^{T} \mid u \text { admissible and }\left\|x\left(T, x_{0}, u\right)-x_{s}\right\| \leq \kappa\right\} .
$$

Then, for problems with terminal constraints (Grüne and Panin, 2015, Thm. 5.2) shows the estimate

$$
J_{T}^{c l}\left(x_{0}, \mu_{N}\right) \leq \inf _{u \in \mathbb{U}_{\kappa}^{T}\left(x_{0}\right)} J_{T}\left(x_{0}, u\right)+\delta_{1}(N)+\delta_{2}(T)
$$

where $\kappa=\beta\left(\left\|x-x_{0}\right\|, T\right)$ with $\beta \in \mathcal{K} L$ from (5.2) and $\delta_{1}, \delta_{2} \in \mathcal{L}$.

For problems without terminal constraints, the estimate changes to $\left\|x(t)-x_{s}\right\| \leq$ $\max \left\{\beta\left(\left\|x-x_{0}\right\|, t\right), \rho\right\}$ with $\rho \rightarrow 0$ as $N \rightarrow \infty$ :

$$
J_{T}^{c l}\left(x_{0}, \mu_{N}\right) \leq \inf _{u \in \mathbb{U}_{\kappa}^{T}(T)} J_{T}\left(x_{0}, u\right)+T \hat{\delta}_{1}(N)+\hat{\delta}_{2}(T)
$$

cf. (Grüne and Stieler, 2014b, Thm. 4.1), where $\kappa=\max \left\{\beta\left(\left\|x-x_{0}\right\|, T\right), \rho(N)\right\}$ with $\beta$ and $\rho$ from $(5.3)$ and $\hat{\delta}_{1}, \hat{\delta}_{2} \in \mathcal{L}$. Thus, similar to the previous section, the difference 


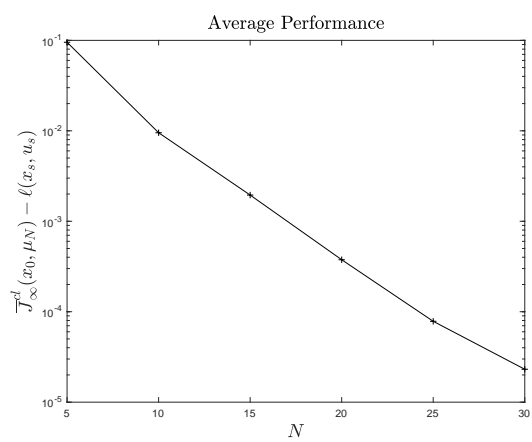

Figure 5.1: $\bar{J}_{\infty}^{c l}\left(x_{0}, \mu_{N}\right)-\ell\left(x_{s}, u_{s}\right)$ without terminal constraints for $N=5, \ldots, 30$.
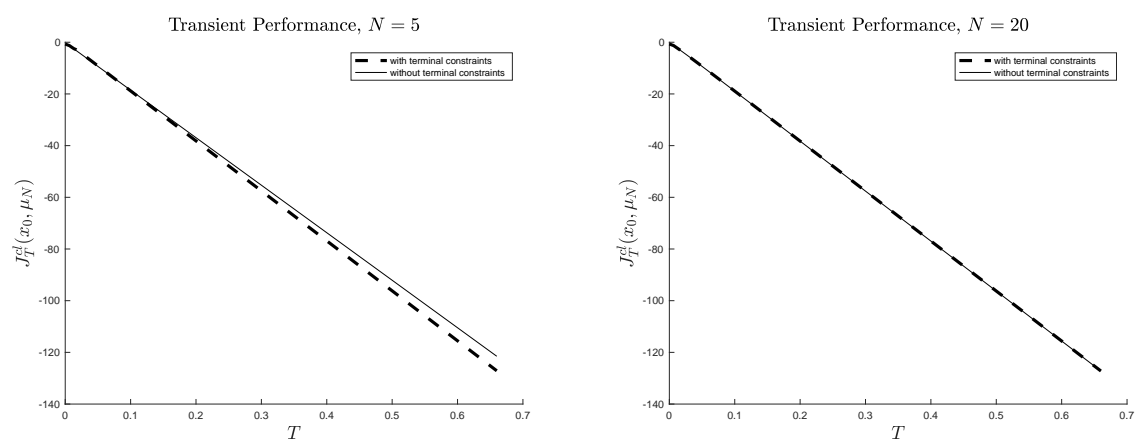

Figure 5.2: $J_{T}^{c l}\left(x_{0}, \mu_{N}\right)$ with equilibrium terminal constraints (solid) $x(N)=x_{s}$ and without terminal constraints (dashed) for $N=5$ (left) and $N=20$ (right).

between the case with and without terminal constraints lies in the fact that without constraints the error term depending on $N$ is multiplied by the length $T$ of the closed loop trajectory piece whose performance is measured.

\subsection{Example - Chemical Reactor with Dissipativity}

We investigate the closed loop performance for the Van de Vusse reactor from Sections 3.3 and 4.4 . The averaged performance of EMPC with terminal constraints is - up to numerical accuracy - identical to $\ell\left(x_{s}, u_{s}\right)$, which confirms the first estimate from Section 5.1. For EMPC without terminal constraints, the second estimate in Section 5.1 suggests that the average performance may be larger than $\ell\left(x_{s}, u_{s}\right)$ but converges to this value for $N \rightarrow \infty$. Figure 5.1 confirms this behaviour. As for the closed-loop equilibrium, cf. Section 4.4, the convergence is exponentially fast, which is a known phenomenon in the literature, see (Grüne and Stieler, 2014b, Remark 4.2(i)).

Regarding the transient performance, the estimates in Section 5.3 imply that there should be a gap between the transient performance with and without terminal constraints 
which grows linearly with $T$. Figure 5.2 (left) shows that this is indeed visible in the numerical simulations for the relatively small horizon $N=5$. For the larger horizon $N=20$ this phenomenon is no longer visible on the time scale of the graph, cf. Figure 5.2 (right), because the term $\hat{\delta}_{1}(N)$ has become very small. 


\section{EMPC with Averaged Constraints}

In this section, we discuss EMPC approaches with additional average constraints. Such constraints on (time) averages of input and state variables naturally arise in the context of EMPC, where the optimal system behavior need not be steady-state operation, cf. Section 3.1 .

Namely, in the context of standard tracking MPC, where the controller is designed such that the closed loop converges to a given equilibrium, any asymptotic average of state or input variables is determined by the value at this equilibrium. This means that such average constraints do not need to be considered online, but have to be taken into account when choosing the setpoint to be stabilized. On the other hand, in the context of EMPC, such average constraints have to be dealt with online, and the repeatedly solved optimization problem has to be modified in a suitable fashion such that the resulting closed-loop system satisfies the given average constraints. Average constraints can be of interest in various applications of EMPC. In particular, this is the case whenever input or output variables are physical entities that allow for storage and retrieval at some later time, such as, e.g., chemical products or various forms of energy. For example, in the process industry, one might want to constrain the average amount of raw material fed into a chemical reactor, or the average heat flux through the reactor wall, compare, e.g., (Lee and Bailey, 1980; Renken, 1972).

In the following, we distinguish between asymptotic and transient average constraints, requiring the constraints to be satisfied over an infinite or finite time horizon, respectively. We first discuss how asymptotic average constraints can be handled, following the exposition in (Angeli et al., 2012; Müller, Angeli, Allgöwer, et al., 2014), before turning to transient average constraints as proposed in (Müller, Angeli, and Allgöwer, 2014b). Note that all of these results are formulated for a setting including terminal constraints as in Section 3, we discuss at the end of this section, how they can be extended to a setting as in Section 4 without such terminal constraints. For the remainder of this section, for technical reasons we assume that $\mathbb{X}$ is compact. 


\subsection{Asymptotic Average Constraints}

First, for any bounded sequence $v: \mathbb{I}_{\geq 0} \rightarrow \mathbb{R}^{n_{v}}$, the set of asymptotic averages is defined as

$$
A v[v]:=\left\{\bar{v} \in \mathbb{R}^{n_{v}}: \exists\left\{t_{n}\right\} \rightarrow+\infty: \lim _{n \rightarrow \infty} \frac{\sum_{k=0}^{t_{n}} v(k)}{t_{n}+1}=\bar{v}\right\}
$$

The definition of $A v[v]$ is such that it contains all accumulation points of the sequence $\frac{\sum_{k=0}^{t} v(k)}{t+1}$. Note that $A v[v]$ is nonempty (as bounded sequences in $\mathbb{R}^{n_{v}}$ have limit points), but it need not be a singleton in general. Asymptotic average constraints are now expressed as

$$
A v[y] \subseteq \mathbb{Y}
$$

where $y=h(x, u)$ is some (auxiliary) output variable, $h: \mathbb{R}^{n} \times \mathbb{R}^{m} \rightarrow \mathbb{R}^{p}$ is continuous, and $\mathbb{Y} \subseteq \mathbb{R}^{p}$ is some closed and convex set. Let $\left(x_{s}, u_{s}\right)$ denote the optimal steady-state which also satisfies the average constraints, defined by

$$
\ell\left(x_{s}, u_{s}\right)=\min _{x \in \mathbb{X}, u \in \mathbb{U}, h(x, u) \in \mathbb{Y}, x=f(x, u)} \ell(x, u)
$$

In order to ensure that the closed-loop system satisfies the average constraints, the repeatedly solved optimization problem is modified as follows:

$$
\min _{u(\cdot \mid t)} \sum_{k=0}^{N-1} \ell(x(k \mid t), u(k \mid t))+V_{f}(x(N \mid t))
$$

subject to

$$
\begin{aligned}
x(k+1 \mid t) & =f(x(k \mid t), u(k \mid t)), & & k=0, \ldots, N-1 \\
x(0 \mid t) & =x(t) & & k=0, \ldots, N-1 \\
(x(k \mid t), u(k \mid t))^{\top} & \in \mathbb{X} \times \mathbb{U}, & & \\
x(N \mid t) & \in \mathbb{X}_{f}(t) & & \\
\sum_{k=0}^{N-1} h(x(k \mid t), u(k \mid t)) & \in \mathbb{Y}_{t} & &
\end{aligned}
$$


Compared to the standard EMPC problem (3.1), OCP 6.3) contains the additional constraint (6.3f) as well as a possibly time-varying terminal region (6.3e) 1 . The time-varying set $\mathbb{Y}_{t}$ is defined recursively as ${ }^{2}$

$$
\begin{aligned}
\mathbb{Y}_{t+1} & :=\mathbb{Y}_{t} \oplus \mathbb{Y} \oplus \overline{\mathbb{Y}}(t) \oplus\{-h(x(t), u(t))\} \\
\mathbb{Y}_{0} & =N \mathbb{Y} \oplus \mathbb{Y}_{00}
\end{aligned}
$$

where $\mathbb{Y}_{00}$ is an arbitrary compact subset of $\mathbb{R}^{p}$ containing $h\left(x_{s}, u_{s}\right)$ and $\bar{Y}(t)$ will be specified later. Note that the recursion in (6.4) can be solved explicitly, which due to convexity of $\mathbb{Y}$ results in

$$
\mathbb{Y}_{t}=\mathbb{Y}_{00} \oplus(t+N) \mathbb{Y} \oplus \sum_{k=0}^{t-1} \overline{\mathbb{Y}}(k) \oplus\left\{\sum_{k=0}^{t-1}-h(x(k), u(k))\right\}
$$

In order to establish desired closed-loop properties, the following assumption is needed, cf. (Müller, Angeli, Allgöwer, et al., 2014, Assumptions 1-3).

\section{Assumption 6.1.}

1. There exists an auxiliary terminal control law $\kappa_{f}: \mathbb{X} \rightarrow \mathbb{U}$ with $\kappa_{f}\left(x_{s}\right)=u_{s}$ and for each $t \in \mathbb{I}_{\geq 0}$ a terminal region $\mathbb{X}_{f}(t)$ such that the following is satisfied for all $x \in \mathbb{X}_{f}(t):$ (i) $\left(x, \kappa_{f}(x)\right) \in \mathbb{X} \times \mathbb{U}$, (ii) $f\left(x, \kappa_{f}(x)\right) \in \mathbb{X}_{f}(t+1)$, and (iii) $V_{f}\left(f\left(x, \kappa_{f}(x)\right)\right)-V_{f}(x) \leq-\ell\left(x, \kappa_{f}(x)\right)+\ell\left(x_{s}, u_{s}\right)$.

2. For each $t \in \mathbb{I}_{\geq 0}$, the set $\overline{\mathbb{Y}}(t)$ is such that $h\left(x, \kappa_{f}(x)\right) \in \mathbb{Y} \oplus \overline{\mathbb{Y}}(t)$ for all $x \in \mathbb{X}_{f}(t)$.

3. The exist a constant $0 \leq \alpha<1$ and a compact set $\overline{\mathbb{Y}}$ such that $\sum_{k=0}^{t-1} \overline{\mathbb{Y}}(k) \subseteq t^{\alpha} \overline{\mathbb{Y}}$ for all $t \in \mathbb{I}_{\geq 0}$.

In case that the terminal region is constant, Assumption 6.1.1 reduces to the standard assumption which is typically employed on the terminal region and cost, both in stabilizing and EMPC (cf. Assumption 2.2). In (Müller, Angeli, Allgöwer, et al., 2014, Section 3.2), a systematic procedure is presented how both the terminal regions $\mathbb{X}_{f}(t)$ as well as the sets $\bar{Y}(t)$ can be determined such that Assumption 6.1 is satisfied. As a special case, the setting of Angeli et al., 2012 is included, where a terminal equality

\footnotetext{
${ }^{1}$ Note that a time-varying terminal region might in particular be necessary if $\left(x_{s}, u_{s}\right)$ is on the boundary of $\mathbb{Y}$. In case that $\left(x_{s}, u_{s}\right) \in \operatorname{int}(\mathbb{Y})$, also a constant terminal region can be used, see (Müller, Angeli, Allgöwer, et al., 2014) for a further discussion of this issue.

${ }^{2}$ The symbol $\oplus$ denontes the Minkowski set addition, which for two sets $A, B \subseteq \mathbb{R}^{n}$ is defined as $A \oplus B:=\left\{a+b \in \mathbb{R}^{n} \mid a \in A, b \in B\right\}$.
} 
constraint $x(N \mid t)=x_{s}$ is used, i.e., $\mathbb{X}_{f}(t) \equiv\left\{x_{s}\right\}$. In this case, one can choose $\overline{\mathbb{Y}}(t) \equiv 0$, and hence Assumption 6.1 is trivially satisfied. Using Assumption 6.1, one can show that the resulting closed-loop system satisfies the asymptotic average constraints (6.1) as desired.

Theorem 6.1. Suppose that Assumption 6.1 is satisfied and OCP (6.3) is feasible at time $t=0$. Then it is feasible for all $t \in \mathbb{N}$ and the resulting closed-loop system satisfies the asymptotic average constraints 6.1.

This theorem was shown in (Angeli et al., 2012) for the special case of terminal equality constraints and later in (Müller, Angeli, Allgöwer, et al., 2014) for the more general setting using Assumption 6.1. The proof of this result uses the same candidate solution as in Section 2.2 (compare 2.8), i.e.,

$$
\tilde{u}(\cdot \mid t+1):=\left\{u^{\star}(1 \mid t), \ldots, u^{\star}(N-1 \mid t), \kappa_{f}\left(x^{\star}(N \mid t)\right)\right\}
$$

with corresponding candidate state sequence

$$
\tilde{x}(\cdot \mid t+1):=\left\{x^{\star}(1 \mid t), \ldots, x^{\star}(N \mid t), f\left(x^{\star}(N \mid t), \kappa_{f}\left(x^{\star}(N \mid t)\right)\right)\right\} .
$$

Using Assumption 6.1.2 and the fact that the constraint 6.3f was satisfied for the optimal solution at time $t$, one obtains

$$
\begin{aligned}
& \sum_{k=0}^{N-1} h(\tilde{x}(k \mid t+1), \tilde{u}(k \mid t+1)) \\
& =\sum_{k=0}^{N-1} h\left(x^{\star}(k \mid t), u^{\star}(k \mid t)\right)+h\left(x^{\star}(N \mid t), \kappa_{f}\left(x^{\star}(N \mid t)\right)\right)-h(x(t), u(t)) \\
& \in \mathbb{Y}_{t} \oplus \mathbb{Y} \oplus \overline{\mathbb{Y}}(t) \oplus\{-h(x(t), u(t))\}=\mathbb{Y}_{t+1}
\end{aligned}
$$

which shows recursive feasibility of the additional constraint $(6.3 \mathrm{f})$. To show satisfaction of the asymptotic average constraints for the closed-loop system, from 6.3f and 6.5 it follows that at any time $t$

$$
\sum_{k=0}^{t-1} h(x(k), u(k))+\sum_{k=0}^{N-1} h(x(k \mid t), u(k \mid t)) \in \mathbb{Y}_{00} \oplus(t+N) \mathbb{Y} \oplus \sum_{k=0}^{t-1} \overline{\mathbb{Y}}(k)
$$

for each predicted input and state sequences $u(\cdot \mid t)$ and $x(\cdot \mid t)$ which are feasible at time $t$. Taking averages on both sides of (6.6) and considering any infinite time sequence 
$\left\{t_{n}\right\}$ such that $\lim _{n \rightarrow+\infty} \frac{\sum_{k=0}^{t_{n}-1} h(x(k), u(k))}{t_{n}}$ exists, the desired result follows from Assumption 6.1.3, compactness of $\mathbb{Y}_{00}, \overline{\mathbb{Y}}, \mathbb{X}$, and $\mathbb{U}$, and continuity of $h$.

Having shown how asymptotic average constraints can be incorporated into a standard EMPC scheme, we now discuss what implications such constraints have on the optimal operating behavior of a system as well as the closed-loop behavior. To this end, in the following we assume that the set $\mathbb{Y}$ in (6.1) is given by $\mathbb{Y}=\left\{y \in \mathbb{R}^{p}: y \leq 0\right\}=\mathbb{R}_{\leq 0}^{p}$. This is not a major restriction since $h$ can be some general nonlinear function. Now recall from Section 3.1 that a system is optimally operated at steady state if it is dissipative with respect to the supply rate $s(x, u)=\ell(x, u)-\ell\left(x_{s}, u_{s}\right)$. In the presence of asymptotic average constraints, a similar result has been obtained in (Angeli et al., 2012, Prop. 6.4). There, it was shown that if the system is dissipative with respect to the supply rate

$$
s(x, u)=\ell(x, u)-\ell\left(x_{s}, u_{s}\right)+\mu^{\top} h(x, u)
$$

for some $\mu \in \mathbb{R}_{\geq 0}^{p}$, then it is optimally operated at steady state on averagely constrained solutions. Here, the latter property means that the asymptotic average performance along each feasible solution, which also satisfies the asymptotic average constraints, is worse or equal to the optimal steady-state cost. The proof of this result is similar to the case without average constraints, using in addition the fact that $A v[h(x, u)] \subseteq \mathbb{R}_{\leq 0}^{p}$ for all feasible state and input sequences satisfying the average constraints. Comparing the supply rate (6.7) with the one used without average constraints (3.3), one can see that the dissipativity condition is relaxed in the region where $h(x, u) \geq 0$ (since $\mu \geq 0$ ), i.e., for those points $(x, u)$ which do not satisfy the constraint $h(x, u) \in \mathbb{Y}$. On the other hand, it is strengthened for all points $(x, u)$ such that $h(x, u) \leq 0$. Since on average $h$ is nonpositive, i.e., $A v[h(x, u)] \subseteq \mathbb{R}_{\leq 0}^{p}$, the relaxation of the dissipativity condition "vanishes on average", resulting again in the fact that steady-state operation is optimal.

In case that steady-state operation is optimal, the following closed-loop convergence result has been obtained in (Müller, Angeli, Allgöwer, et al., 2014).

Theorem 6.2. Suppose that Assumption 6.1 is satisfied with $\alpha=0$, OCP $(6.3)$ is feasible at time $t=0$, and the system is strictly dissipative with respect to the supply rate $s(x, u)=\ell(x, u)-\ell\left(x_{s}, u_{s}\right)+\mu^{\top} h(x, u)$ for some $\mu \in \mathbb{R}_{\geq 0}^{p}$. Then the resulting closed-loop system asymptotically converges to $x_{s}$, i.e., $\lim _{t \rightarrow \infty} x(t)=x_{s}$.

In order to prove this result, a different Lyapunov function than in Section 3.2 has to be employed. Namely, denote again by $\widetilde{V}_{N}^{\star}(x(t))$ the optimal value function of ocP $(6.3)$ with $\ell$ and $V_{f}$ in $6.3 \mathrm{a}$ replaced by the rotated stage and terminal cost functions $\tilde{\ell}$ and $\widetilde{V}_{f}$, 
respectively. As was shown in Lemma 3.2, the solution to this modified optimization problem is identical to the solution of the original ocP $(6.3)$, since the cost functions only differ by a constant term and the constraints are the same. We now use the following Lyapunov-like function in order to establish closed-loop convergence:

$$
V(t)=\widetilde{V}_{N}^{\star}(x(t))+w(t)
$$

with

$$
w(t):=\sup _{T \geq 0} \sum_{k=t}^{t+T} \mu^{\top} y(k) .
$$

Here, the sequence $y(\cdot)$ is the output along the solution of the closed loop from time $t$ on. Using 6.6), Assumption 6.1.3 with $\alpha=0$, compactness of $\mathbb{Y}_{00}, \overline{\mathbb{Y}}, \mathbb{X}$, and $\mathbb{U}$, and continuity of $h$, one can show that $w(0)$ is upper and lower bounded by some finite constant, for each feasible initial condition $x_{0}$. The same is true for $\widetilde{V}_{N}^{\star}(x(0))$ and hence also for $V(0)$. As shown in Sections 2.2 and 3.2 (compare the proofs of Theorems 2.1 and 3.2. we have $\widetilde{V}_{N}^{\star}(x(t+1))-\widetilde{V}_{N}^{\star}(x(t)) \leq-L\left(x(t), u^{\star}(0 \mid t)\right)$. Hence, using the strict dissipativity assumption as well as the definition of $w$ in $(6.9$, one obtains

$$
\begin{aligned}
& V(t+1)-V(t) \leq-L\left(x(t), u^{\star}(0 \mid t)\right)+w(t+1)-w(t) \\
& \leq-\alpha\left(\left|x(t)-x_{s}\right|\right)+\mu^{\top} h\left(x(t), u^{\star}(0 \mid t)\right)+w(t+1)-w(t) \\
& =-\alpha\left(\left|x(t)-x_{s}\right|\right)+\mu^{\top} h\left(x(t), u^{\star}(0 \mid t)\right) \\
& \quad+\sup _{T \geq 0} \sum_{k=t+1}^{t+1+T} \mu^{\top} h\left(x(k), u^{\star}(0 \mid k)\right)-\sup _{T \geq 0} \sum_{k=t}^{t+T} \mu^{\top} h\left(x(k), u^{\star}(0 \mid k)\right) \\
& =-\alpha\left(\left|x(t)-x_{s}\right|\right)+\sup _{T \geq 1} \sum_{k=t}^{t+T} \mu^{\top} h\left(x(k), u^{\star}(0 \mid k)\right) \\
& \quad-\sup _{T \geq 0} \sum_{k=t}^{t+T} \mu^{\top} h\left(x(k), u^{\star}(0 \mid k)\right) \\
& \leq-\alpha\left(\left|x(t)-x_{s}\right|\right) .
\end{aligned}
$$

Since $V$ is bounded from above and below and nonincreasing, it converges. By 6.10, this implies that $x(t)$ converges to $x_{s}$ as $t \rightarrow \infty$, concluding the proof.

We note that in contrast to the setting without average constraints (compare Theorem 3.2 , in general only asymptotic convergence of the closed loop to $x_{s}$ can be estab- 


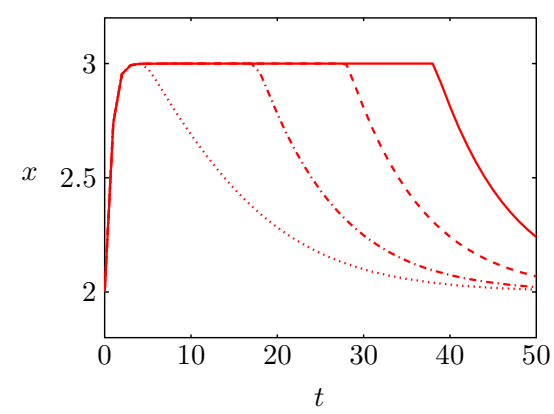

Figure 6.1: Closed-loop state sequences of system (6.11) with $\bar{y}=20$ (dotted), $\bar{y}=40$ (dash-dotted), $\bar{y}=60$ (dashed), and $\bar{y}=80$ (solid).

lished, but not asymptotic stability of $x_{s}$. This is due to the fact that the asymptotic average constraints allow the system to "spend time" in a region of the state space where it is not allowed on average and where the cost is lower than at the optimal steady-state $x_{s}$. This behavior is illustrated with the following simple example, taken from (Müller, Angeli, Allgöwer, et al., 2014).

\subsection{Simple Example}

Consider the system

$$
x(t+1)=x(t) u(t)
$$

with state and input constraint set $\mathbb{X}=\mathbb{U}=[-10,10]$ and average constraint of the form 6.1 with $y=h(x, u)=2 x+u-5$ and $\mathbb{Y}=\mathbb{R}_{\leq 0}$. The stage cost is given by $\ell(x, u):=(x-3)^{2}+u^{2}$, resulting in the optimal steady-state $\left(x_{s}, u_{s}\right)=(2,1)$. One can show that the system is strictly dissipative with respect to the supply rate $s(x, u)=\ell(x, u)-\ell\left(x_{s}, u_{s}\right)+\mu h(x, u)$ with $\mu=1$ and storage function $\lambda(x)=1.5 x+c_{1}$ for some $c_{1} \in \mathbb{R}_{\geq 0}$. Figure 6.1 shows closed-loop state and input sequences with prediction horizon $N=10$ and $\mathbb{Y}_{00}=\{y \in \mathbb{R}: y \leq \bar{y}\}$ for different values of $\bar{y}$. As guaranteed by Theorem 6.2. the closed-loop system converges to $x_{s}$. However, $x_{s}$ is not a Lyapunov stable equilibrium point, since the closed-loop first moves away from $x_{0}=x_{s}=2$ and approaches $x=3$, before finally converging to $x_{s}=2$. In fact, it turns out that without the average constraint, the optimal steady-state would be given by $(x, u)=(3,1)$ with $\ell(3,1)<\ell(2,1)$, and the system would be strictly dissipative with respect to the supply rate $s(x, u)=\ell(x, u)-\ell(3,1)$ and storage function $\lambda(x)=(2 / 3) x+c_{2}$ for some $c_{2} \in \mathbb{R}_{\geq 0}$. This means that the closed-loop behavior with average constraints is such that the system 
initially converges to the optimal operating behavior without average constraint, i.e., to $x=3$. After some transient phase, the system is forced to leave this steady-state such that the asymptotic average constraints can be satisfied. The amount of time the system is allowed to stay in a vicinity of $x=3$ can be tuned by choosing the parameter $\bar{y}$ in $\mathbb{Y}_{00}$ accordingly (see Figure 6.1).

\subsection{Transient Average Constraints}

The above results show how asymptotic average constraints can be incorporated into EMPC schemes. In other situations, rather transient average constraints are of interest, meaning that state and input values averaged over some finite time horizon $T$ should be constrained. This means that for all $t \in \mathbb{I}_{\geq 0}$, the following should be satisfied:

$$
\sum_{k=t}^{t+T-1} \frac{h(x(k), u(k))}{T} \in \mathbb{Y}
$$

where $h: \mathbb{R}^{n} \times \mathbb{R}^{m} \rightarrow \mathbb{R}^{p}$ is again some auxiliary output map which is assumed to be continuous. One method how such transient average constraints can be incorporated into EMPC was shown in (Müller, Angeli, and Allgöwer, 2014b), again for the case where $\mathbb{Y}=\mathbb{R}_{\leq 0}^{p}$. Namely, instead of incorporating the constraint (6.3f), the following constraints are added to the repeatedly solved optimization problem:

$$
\begin{aligned}
& \sum_{k=t-T+i}^{t-1} h(x(k), u(k))+\sum_{k=0}^{i-1} h(x(k \mid t), u(k \mid t)) \leq 0, \\
& i=\max \{1, T-t\}, \ldots, T \\
& \sum_{k=j}^{j+T-1} h(x(k \mid t), u(k \mid t)) \leq 0, \quad j=1, \ldots, N-1 \\
& u(k \mid t)=\kappa_{f}(x(k \mid t)), \quad k=N, \ldots, N+T-2
\end{aligned}
$$

These additional constraints can be interpreted as follows. Constraint (6.13) ensures that past state and input values together with predicted ones satisfy the transient average constraints over each time window $T$, while constraint 6.14 does the same for predicted states and inputs further in the future. In order to be able to ensure recursive feasibility, predictions up to $N+T-2$ steps into the future are needed, i.e., beyond the prediction horizon $N$. However, predictions farther than $N$ steps in the future are 
fixed by 6.15), i.e., the horizon over which one optimizes (and hence the number of optimization variables) stays the same. Once recursive feasibility is established, transient average constraint satisfaction for the resulting closed-loop system directly follows from 6.13) with $i=1$. Furthermore, if steady-state operation is optimal, closed-loop convergence to the optimal steady-state $x_{s}$ can again be ensured if the system is strictly dissipative with respect to the supply rate (6.7), using a similar proof as above in case of asymptotic average constraints.

\subsection{Extensions}

The above results have been obtained in a setting with terminal constraints. If instead an EMPC scheme without such terminal constraints shall be used, asymptotic and transient average constraint satisfaction for the resulting closed-loop system can be ensured in the same fashion, i.e., by including the constraints (6.3f) and 6.4 or 6.13) 6.15), respectively, into the repeatedly solved optimization problem. This is the case since the above proof of closed-loop average constraint satisfaction, given recursive feasibility, does not depend on the presence of terminal constraints. The problem, however, is how recursive feasibility can be ensured in case of no terminal constraints. Namely, the additional constraints (6.3f) and (6.4) or 6.13)-(6.15), respectively, are time-varying. Hence assuming control invariance of $\mathbb{X}$, which immediately gives recursive feasibility in case of no average constraints, is in general not sufficient anymore.

Establishing recursive feasibility of EMPC without terminal constraints and including average constraints, maybe based on turnpike arguments similar to Section 4.2, is still open and an interesting subject of future work. Concerning average performance, one can show in the same fashion as in case without average constraints that the asymptotic average performance of the closed loop is upper bounded by $\ell\left(x^{s}, u^{s}\right)$, using Assumption 6.1.1. On the other hand, establishing transient performance estimates in the presence of average constraints is also an open problem, which is currently under investigation. Here, exploiting two turnpike properties (one without and one with average constraints) might be helpful, similar to the situation observed in the example of Section 6.2. 



\section{EMPC with Generalized Terminal Constraints}

In this section, we discuss EMPC approaches using a generalized terminal constraint. Compared to the results in Section 3, this means that the predicted terminal state has to be equal to some (arbitrary) steady-state instead of the optimal steady-state, which in general leads to a (significantly) larger feasible region. Using such generalized terminal constraints was first proposed in (Ferramosca, Limon, Alvarado, et al., 2009; Limon, Alvarado, et al., 2008) in the context of stabilizing (tracking) MPC and has later also been employed in EMPC by, e.g., Fagiano and Teel, 2013, Ferramosca, Limon, and Camacho, 2014 and Müller, Angeli, and Allgöwer, 2013. These approaches differ in how the underlying optimal control problem is formulated (in particular, which terminal cost is used) and how / under what conditions closed-loop guarantees can be given, but the main idea of using a generalized terminal constraint instead of a fixed one is the same. In the following, we will mainly concentrate on the formulation used in (Fagiano and Teel, 2013; Müller, Angeli, and Allgöwer, 2013), and we also comment on differences and available further results in other references. 


\subsection{Problem Formulation and Performance Analysis}

When considering generalized terminal constraints, the optimal control problem solved at each time $t$ with $x:=x(t)$ is given as follows:

$$
\min _{u(\cdot \mid t)} \sum_{k=0}^{N-1} \ell(x(k \mid t), u(k \mid t))+\beta \ell(x(N \mid t), u(N \mid t))
$$

subject to

$$
\begin{aligned}
x(0 \mid t) & =x & & \\
x(k+1 \mid t) & =f(x(k \mid t), u(k \mid t)) & & k=0, \ldots, N-1 \\
(x(k \mid t), u(k \mid t))^{\top} & \in \mathbb{X} \times \mathbb{U}, & & k=0, \ldots, N \\
x(N \mid t) & =f(x(N \mid t), u(N \mid t)), & & \\
\ell(x(N \mid t), u(N \mid t)) & \leq \kappa(t) . & &
\end{aligned}
$$

for some $\beta>0$. Denote by $\mathbb{X}_{N}^{g e n}$ the set of all states $x \in \mathbb{X}$ for which ocP $(7.1)$ has a solution (for some $\kappa$ ). The special feature of this optimization problem is the generalized terminal constraint in $7.1 \mathrm{e}$, meaning that the predicted terminal state $x(N \mid t)$ has to be equal to an arbitrary steady state and not necessarily to the optimal one (or inside a terminal set around it) as in Section 3 .

Remark 7.1 (Advantages of generalized terminal constraints). The main benefits of using such a generalized terminal constraint compared to a fixed terminal constraint are that (i) in general a possibly much larger feasible region can be obtained and (ii) recursive feasibility is maintained if the cost function (and hence also possibly the optimal steady-state) changes online. On the other hand, the number of optimization variables is slightly increased, and the closed-loop analysis becomes more involved and (slightly) weaker results can be established.

The parameter $\kappa$ in $(7.1 \mathrm{f})$ is updated according to the cost of the previous terminal state, i.e., the following closed-loop system is obtained:

$$
\begin{aligned}
& x(t+1)=f\left(x(t), u^{\star}(0 \mid t)\right), \\
& \kappa(t+1)=\ell\left(x^{\star}(N \mid t), u^{\star}(N \mid t)\right),
\end{aligned}
$$

with an appropriate (large enough) initialization of $\kappa$. The constraint (7.1f) together with $(7.2)$ ensures that the sequence of predicted terminal steady states $(x(N \mid \cdot), u(N \mid \cdot))$ has a nonincreasing stage cost. Since $\kappa(t)$ is nonincreasing and bounded from below (by 
$\left.\ell\left(x_{s}, u_{s}\right)\right)$, it converges. Denote the limit by $\kappa_{\infty}:=\lim _{t \rightarrow \infty} \kappa(t)$. Note that the sequence $\kappa(\cdot)$ is convergent irrespective of the evolution of the terminal weight $\beta$, however, the limit $\kappa_{\infty}$ does in general depend on $\beta$, as discussed in the following.

Similar to Section 5.1 (compare (5.1)), one can show that the closed-loop asymptotic average performance (for fixed terminal weight $\beta$ ) is upper bounded by

$$
J_{\infty}^{c l}\left(x_{0}, \mu_{N}\right) \leq \kappa_{\infty}
$$

Hence the study of the limiting behavior of $\kappa(\cdot)$ is of key interest and a small value of $\kappa_{\infty}$ would be desirable. In order to give bounds on $\kappa_{\infty}$, we first study the effect of the terminal weight $\beta$ on the predicted terminal state $x(N \mid t)$. Intuitively, a larger value of $\beta$ is expected to result in a terminal steady state $(x(N \mid t), u(N \mid t))$ with a lower cost $\ell(x(N \mid t), u(N \mid t))$. This can formally be shown as follows. Denote the set of steady-states which are reachable in $N>0$ steps from a point $y \in \mathbb{X}$ by

$$
\begin{aligned}
\mathcal{X}_{N}(y):= & \left\{x \in \mathbb{X}: \exists u \in \mathbb{U}^{(N+1)} \text { s.t. } x(0)=y,\right. \\
& x(j+1)=f(x(j), u(j)), 0 \leq j \leq N-1, x(N)=x, \\
& x=f(x, u(N)),(x(j), u(j)) \in \mathbb{X} \times \mathbb{U}, 0 \leq j \leq N\} .
\end{aligned}
$$

Next, define the best achievable steady-state cost from a point $y \in \mathbb{X}$ as ${ }^{1}$

$$
\begin{aligned}
\ell_{\min }(y) & :=\min _{x, u} \ell(x, u) \\
\text { s.t. } & x \in \mathcal{X}_{N}(y), u \in \mathbb{U}, x=f(x, u) .
\end{aligned}
$$

Furthermore, define the best robustly achievable steady-state cost from a point $y \in \mathbb{X}$ as follows. For each $\varepsilon \geq 0$, let

$$
\ell_{\min }(y, \varepsilon):=\sup _{z \in B_{\varepsilon}(y) \cap \mathbb{X}} \ell_{\min }(z) .
$$

With this, we define the best robustly achievable steady-state cost from a point $y \in \mathbb{X}$ as

$$
\bar{\ell}_{\min }(y):=\lim _{\varepsilon \searrow 0} \ell_{\min }(y, \varepsilon)
$$

The limit in (7.7) exists since $\ell_{\min }(y, \varepsilon)$ is monotonically nonincreasing as $\varepsilon \searrow 0$. It

\footnotetext{
${ }^{1}$ By convention, the minimum is $+\infty$ if it is taken over the empty set.
} 
immediately follows from the definitions in (7.5) and (7.7) that for each $y \in \mathbb{X}$ we have $\ell_{\min }(y) \leq \bar{\ell}_{\min }(y)$. However, equality does not hold in general since $\ell_{\min }(y, \varepsilon)$ is not necessarily continuous in $\varepsilon$ at $\varepsilon=0$ (see, e.g., Müller, Angeli, and Allgöwer, 2013, Example 4). Given the above definitions, we can now state the following result, which has been obtained in (Fagiano and Teel, 2013, Prop. 2).

Theorem 7.1 (EMPC with constant terminal weight). Suppose that $\mathbb{X}$ is compact and let $\varepsilon>0$. There exists $\underline{\beta}(\varepsilon)>0$ such that for all $\beta \geq \underline{\beta}(\varepsilon)$, all $x(t) \in \mathbb{X}_{N}^{\text {gen }}$ and $\kappa(t) \geq \ell_{\min }(x(t))+\varepsilon$, the solution to OCP $(7.1)$ is such that

$$
\ell\left(x^{\star}(N \mid t), u^{\star}(N \mid t)\right) \leq \ell_{\min }(x(t))+\varepsilon .
$$

Theorem 7.1 says that if the terminal weight $\beta$ is large enough, the cost of the predicted terminal steady-state is close to the best achievable steady-state cost. This result can be proven by contradiction as follows. Consider a feasible input sequence $\bar{u}(\cdot \mid t)$ to OCP (7.1) with corresponding state sequence $\bar{x}(\cdot \mid t)$ such that $\ell(\bar{x}(N \mid t), \bar{u}(N \mid t))=$ $\ell_{\min }(x(t))$. Now assume for contradiction that the optimal solution to oCP $(7.1)$ is such that $\ell\left(x^{\star}(N \mid t), u^{\star}(N \mid t)\right)>\ell_{\min }(x(t))+\varepsilon$. Due to compactness of $\mathbb{X}$ and $\mathbb{U}$ and continuity of $f$ and $\ell$, there exists some $\eta>0$ such that

$$
\begin{aligned}
& J_{N}(x(t), \bar{u}(\cdot \mid t))-J_{N}\left(x(t), u^{\star}(\cdot \mid t)\right) \\
& =\beta\left(\ell_{\min }(x(t))-\ell\left(x^{\star}(N \mid t), u^{\star}(N \mid t)\right)\right) \\
& \quad+\sum_{k=1}^{N-1}\left(\ell(\bar{x}(N \mid t), \bar{u}(N \mid t))-\ell\left(x^{\star}(N \mid t), u^{\star}(N \mid t)\right)\right) \\
& <-\beta \varepsilon+\eta,
\end{aligned}
$$

for all $x(t) \in \mathbb{X}_{N}^{g e n}$. Choosing $\beta \geq \eta / \varepsilon=: \underline{\beta}(\varepsilon)$ contradicts optimality of $u^{\star}$ and hence proves the theorem.

Theorem 7.1 can now be used to obtain bounds on the closed-loop evolution of the predicted terminal steady-state cost $\ell\left(x^{\star}(N \mid \cdot), u^{\star}(N \mid \cdot)\right)$ and in particular of $\kappa_{\infty}$. A first such result was obtained in (Fagiano and Teel, 2013, Thm. 3) under a certain controllability assumption and for a modified MPC scheme. Namely, assume that there exists some $N^{\prime} \in \mathbb{I}_{\geq 0}$ and $\varepsilon>0$ such that from each steady state $(x, u) \in$ $\mathbb{X} \times \mathbb{U}$, one can reach a "better" steady state $(\bar{x}, \bar{u})$ in $N^{\prime}$ steps, i.e., $\bar{x} \in \mathcal{X}_{N^{\prime}}(x)$ and $\ell(\bar{x}, \bar{u}) \leq \max \left\{\ell\left(x_{s}, u_{s}\right), \ell(x, u)-\varepsilon\right\}$. Given this assumption, a prediction horizon $N \geq N^{\prime}$ has to be chosen and the MPC scheme is modified as follows. At each time $t$, 
if $\left.\left.\ell\left(x^{\star}(N \mid t), u^{\star}(N \mid t)\right)\right)>\ell\left(x^{\star}(N \mid t-1), u^{\star}(N \mid t-1)\right)\right)-\varepsilon$ and $\left.\ell\left(x^{\star}(N \mid t), u^{\star}(N \mid t)\right)\right)>$ $\ell\left(x_{s}, u_{s}\right)+\varepsilon$, i.e., if the predicted terminal steady-state cost does not decrease by at least $\varepsilon$ compared to the previous time step and is not already close to the (global) optimum $\ell\left(x_{s}, u_{s}\right)$, then the new optimal solution $u^{\star}(\cdot \mid t)$ is discarded and the next step $u^{\star}(1 \mid t-1)$ of the solution from the previous time step $t-1$ is applied. This modification results in the fact that during each $N^{\prime}$ time steps, the predicted terminal steady-state cost decreases by at least $\varepsilon$ or (if the optimal solution is repeatedly discarded) the terminal steady-state is reached after $N^{\prime}$ steps, from which by the controllability assumption a better steady state can be reached. In the latter case, using Theorem 7.1, a decrease in the predicted terminal steady-state cost is guaranteed. Summarizing the above, existence of a finite time $T^{\prime}$ is guaranteed such that the predicted terminal steady-state cost satisfies

$$
\ell\left(x^{\star}\left(N \mid T^{\prime}\right), u^{\star}\left(N \mid T^{\prime}\right)\right) \leq \ell\left(x_{s}, u_{s}\right)+\varepsilon,
$$

and hence $\kappa_{\infty} \leq \ell\left(x_{s}, u_{s}\right)+\varepsilon$.

\subsection{Self-tuning Terminal Weight}

In case that the above controllability condition is not satisfied, a shorter prediction horizon $N<N^{\prime}$ is used, or the original MPC scheme without modification is applied, the above upper bound for $\kappa_{\infty}$ cannot be guaranteed in general. In this case, one can use a suitable self-tuning, adaptive terminal weight $\beta$ in order to obtain an upper bound on $\kappa_{\infty}$ as was done in (Müller, Angeli, and Allgöwer, 2014a; Müller, Angeli, and Allgöwer, 2013). Namely, assume that $\beta$ evolves according to some update rule

$$
\beta(t+1)=B(\beta(t), x(t), \kappa(t)), \quad \beta(0)=\beta_{0} \geq 0
$$

Now let $\omega_{B}\left(x_{0}\right)$ be the $\omega$-limit set of the closed-loop state sequence $(7.2)$ starting at $x_{0}$ and using the update rule $B\left(7.9\right.$, i.e., $\omega_{B}\left(x_{0}\right):=\left\{y \in \mathbb{X}: \exists\left\{t_{n}\right\} \rightarrow+\infty\right.$ s.t $x(0)=$ $x_{0}$ and $\left.\lim _{n \rightarrow \infty} x\left(t_{n}\right)=y\right\}$, where $x(\cdot)$ is the closed-loop solution arising from 7.2 and (7.9). The following result has been obtained in (Müller, Angeli, and Allgöwer, 2013, Thm. 2).

Theorem 7.2 (EMPC with self-tuning terminal weight).

(i) Suppose that the update rule $B$ is such that for all sequences $x(\cdot)$ and $\kappa(\cdot)$, regarded 
as open-loop input signals in (7.9), it holds that

$$
\kappa_{\infty}-\liminf _{t \rightarrow \infty} \ell_{\min }(x(t))>0 \Rightarrow \liminf _{t \rightarrow \infty} \beta(t)=\infty .
$$

Then, for the closed-loop system (7.2) and (7.9), it holds that the $\operatorname{limit}_{t \rightarrow \infty} \ell_{\min }(x(t))$ exists and

$$
\kappa_{\infty}=\lim _{t \rightarrow \infty} \ell_{\min }(x(t)) \leq \inf _{y \in \omega_{B}\left(x_{0}\right)} \bar{\ell}_{\min }(y)
$$

(ii) Suppose that the update rule $B$ is such that for all sequences $x(\cdot)$ and $\kappa(\cdot)$, regarded as open-loop input signals in (7.9), it holds that

$$
\kappa_{\infty}-\limsup _{t \rightarrow \infty} \ell_{\min }(x(t))>0 \Rightarrow \limsup _{t \rightarrow \infty} \beta(t)=\infty
$$

Then, for the closed-loop system (7.2) and (7.9), it holds that

$$
\kappa_{\infty}=\limsup _{t \rightarrow \infty} \ell_{\min }(x(t)) \leq \sup _{y \in \omega_{B}\left(x_{0}\right)} \bar{\ell}_{\min }(y)
$$

The intuition behind this result is as follows. The update rule $B$ should be such that if the predicted terminal steady-state cost $\ell\left(x^{\star}(N \mid t), u^{\star}(N \mid t)\right)$ is "large" compared to the best achievable steady-state cost $\ell_{\min }(x(t))$, the terminal weight $\beta$ should be increased in order to ensure a better terminal steady-state cost. This property is encoded by conditions 7.10 and 7.12 , respectively. Using these conditions, the equalities in (7.11) and 7.13), respectively, can be proven by contradiction using Theorem 7.1. The inequalities in (7.11) and (7.13), respectively, then follow from the definitions of the best robustly achievable steady-state cost $\bar{\ell}_{\min }$ and the $\omega$-limit set $\omega_{B}$. In (Müller, Angeli, and Allgöwer, 2013), six different update rules are presented such that the assumptions of Theorem 7.2 are satisfied, out of which we exemplarily show the following two. To this end, define $\delta(t):=\ell\left(x^{\star}(N \mid t), u^{\star}(N \mid t)\right)-\ell_{\min }(x(t))$ and let $\alpha_{1}, \alpha_{2}, \alpha_{3} \in \mathcal{K}$.

$$
\begin{aligned}
& B_{1}(\beta(t), x(t), \kappa(t)):=\beta(t)+\alpha_{1}(\delta(t)) \\
& B_{2}(\beta(t), x(t), \lambda(t)):= \begin{cases}1 & \text { if } C_{3}(t) \leq 0, \\
\beta(t)+\alpha_{2}(\delta(t)) & \text { else }\end{cases}
\end{aligned}
$$

Here, $C_{3}(0)=0$ and for each $t \in \mathbb{I}_{\geq 1}, C_{3}(t):=\ell\left(x^{\star}(N \mid t), u^{\star}(N \mid t)\right)-\ell\left(x^{\star}\left(N \mid t_{\text {last }}\right), u^{\star}\left(N \mid t_{\text {last }}\right)\right)+$ $\alpha_{3}(\delta(t))$ with $t_{\text {last }}:=\max _{s \leq t, \beta(s)=1} s-1$. Update rule $B_{1}$ is such that the terminal 
weight $\beta$ is increased whenever the difference between the predicted terminal steadystate cost and the best achievable steady-state cost is nonzero, while the second also allows for resets of $\beta$ in order to avoid unnecessarily large values of $\beta$, which might be bad for (transient) performance and numerical reasons. One can show that update rule $B_{1}$ is such that the stronger condition 7.10 is satisfied, while for update rule $B_{2}$ 7.12 holds. One drawback of both update rules is that the best achievable steady-state cost has to be known in each time step. For different update rules avoiding this as well as for a further discussion on the different properties of the update rules, the interested reader is referred to (Müller, Angeli, and Allgöwer, 2013).

\subsection{Discussion and Extensions}

Theorem 7.2 together with 7.3 guarantees that the closed-loop average performance is no worse than the cost of the best steady-state achievable from the $\omega$-limit set of the resulting closed-loop trajectory. This is a result of rather conceptual nature. More explicit, a priori verifiable bounds for $\kappa_{\infty}$ can be obtained if instead of the generalized terminal equality constraint (7.1e), a generalized terminal region constraint is used. If this generalized terminal region is constructed appropriately, one can show that $\kappa_{\infty}$ converges to a local minimum of the stage cost on the set of feasible steady states, i.e., to a local optimum of Problem 3.4, see (Müller, Angeli, and Allgöwer, 2014a, Thm. 3). In case that the system is linear with convex stage cost and constraints, $\kappa_{\infty}$ converges to the global minimum of the stage cost on the set of feasible steady states, recovering the results of Section 5.1 .

The previous results show how different asymptotic average performance guarantees can be obtained when using EMPC schemes with generalized terminal constraints. On the other hand, only few closed-loop convergence results as well as transient performance results are, if at all, available in the literature. The work of Ferramosca, Limon, and Camacho, 2014 establishes asymptotic stability of the optimal steady state $x_{s}$ under a strong duality condition for linear systems. This condition can be seen as a special case of the dissipativity condition of Section 3.1 with a linear storage function. As mentioned above, while the main idea of using a generalized terminal constraint is the same as in the above references, the employed cost function in (Ferramosca, Limon, and Camacho, 2014 ) is slightly different compared to OCP (7.1). For the general nonlinear case, proving closed-loop asymptotic stability of the optimal steady-state given the dissipativity condition $3.2 \mathrm{~b})$ is not as straightforward as in the case with a fixed terminal constraint, since 
the modified optimization problem using the rotated cost function does not necessarily result in the same solution as the original problem. In order to establish (practical) asymptotic stability, we expect that techniques based on the turnpike property (similar to the case without terminal constraints) could be used. Also, establishing transient performance results similar to Section 5.3 is still an open problem.

The above results using a generalized terminal constraint have been extended in (Limon, Pereira, et al., 2014) to the case of periodic linear systems with periodic stage cost functions. Here, a cost function is used which penalizes the distance to some periodic trajectory together with a cost term which consists of the real (economic) cost of this periodic trajectory. In (Houska and Müller, 2017), a slightly different type of generalized periodic terminal constraints is used, namely a periodic return constraint requiring that the terminal predicted state $x(N \mid t)$ is equal to the current state $x(t)$. An advantageous property of the latter scheme is that the optimal period length does not have to be known a priori; on the other hand, typically only closed-loop convergence to a locally optimal $N$-periodic orbit can be shown, but not necessarily to the (globally) optimal periodic orbit. Finally, the work (Broomhead et al., 2015) uses generalized periodic terminal constraints in a setting with linear systems subject to disturbances, and robust stability of the optimal periodic orbit is again established under a strong duality assumption. 


\section{Lyapunov-based Approach}

As we have seen in the example of Section 3.4, whenever dissipativity does not hold, the closed loop solutions resulting from EMPC will not in general converge to an equilibrium or stay in the neighborhood of an equilibrium. However, for various reasons convergence to a prescribed equilibrium or at least keeping the state in the basin of attraction of an equilibrium may be desirable, while at the same time the given economic cost should be minimized. This is the task for which Lyapunov-basedEMPC has been designed. While originally developed in continuous time, see (Heidarinejad et al., 2012), here we will stay within the general framework of this survey and present the algorithm and the basic results for discrete time systems. The original continuous time formulation will briefly be explained afterwards in Remark 8.2 ,

\subsection{Basics of the Scheme}

For introducing this method, we need the knowledge of a Lyapunov function and a corresponding controller according to the following definition. In order to avoid notational confusion with the optimal value functions used in other places in this survey, we use the symbol " $W$ " (instead of the more common " $V$ ") for the Lyapunov function.

Definition 8.1 (Controller-specific Lyapunov function). Let $x_{s} \in \mathbb{X}$ be an equilibrium of $f$, i.e., there is $u_{s} \in \mathbb{U}$ with $f\left(x_{s}, u_{s}\right)=x_{s}$. Let $O$ be an open neighborhood of $x_{s}$ and consider two functions $W: O \rightarrow \mathbb{R}$ and $h: O \rightarrow \mathbb{U}$ with $h(x) \in \mathbb{U}$ and $f(x, h(x)) \in O$ for all $x \in O$. Then we say that $W$ is a Lyapunov function with respect to the controller $h$ if there are functions $\alpha_{1}, \alpha_{2}, \alpha_{3} \in \mathcal{K}_{\infty}$ such that the inequalities

$$
\alpha_{1}\left(\left|x-x_{s}\right|\right) \leq W(x) \leq \alpha_{2}\left(\left|x-x_{s}\right|\right)
$$

and

$$
W(f(x, h(x))) \leq W(x)-\alpha_{3}\left(\left|x-x_{s}\right|\right)
$$

hold for all $x \in O$. 
It follows from standard Lyapunov function arguments (see, e.g., (Grüne and Pannek, 2017, Thm. 2.19)) that if a Lyapunov function $W$ and a corresponding controller $h$ exist, then $x_{s}$ is asymptotically stable with basin of attraction $O$ for the closed loop system

$$
x^{+}=f(x, h(x)) .
$$

Note that if $O$ is a level set

$$
\Omega_{\rho}:=\left\{x \in \mathbb{R}^{n} \mid W(x) \leq \rho\right\}
$$

of a Lyapunov function $W$ defined on the whole $\mathbb{R}^{n}$ and satisfying (8.1) for all $x \in \mathbb{R}^{n}$, then the condition $f(x, h(x)) \in O$ readily follows from (8.2).

The idea of Lyapunov-based EMPC is now to use the decrease condition (8.2) as a constraint in the EMPC optimization. More precisely, the original algorithm proposed in (Heidarinejad et al., 2012) uses two operation modes: in the first mode, which is active until a time $t^{\prime}$, the algorithms uses the Lyapunov function $W$ in order to ensure that the system first enters and then stays in a level set $\Omega_{\tilde{\rho}}$ for some $\tilde{\rho}>0$. After the time $t^{\prime}$, $W$ is used in order to ensure convergence of the closed loop solution to $x_{s}$. Note that $t^{\prime}$ may be infinite, in which case the scheme stays in the first mode forever.

The resulting optimal control problem to be solved in each step of the EMPC algorithm then reads as follows.

$$
\min _{u(\cdot \mid t)} \sum_{k=0}^{N-1} \ell(x(k \mid t), u(k \mid t))
$$

subject to

$$
\begin{array}{rlrl}
x(k+1 \mid t) & =f(x(k \mid t), u(k \mid t)), & & k=0, \ldots, N-1 \\
x(0 \mid t) & =x(t) & & \\
(x(k \mid t), u(k \mid t))^{\top} & \in \mathbb{X} \times \mathbb{U}, & k=0, \ldots, N-1 \\
W(x(k \mid t)) & \leq \tilde{\rho}, \quad k=0, \ldots, N \quad \text { if } t \leq t^{\prime} \text { and } W(x(t)) \leq \tilde{\rho} \\
W(x(1 \mid t)) & \leq W\left(f(x(t), h(x(t))) \text { if } t>t^{\prime} \text { or } W(x(t))>\tilde{\rho}\right.
\end{array}
$$

Here the constraints 8.3e and 8.3f contain the implicit constraints $x(k, t) \in O$ and $x(1, t) \in O$, respectively, in order to ensure that $W$ is defined at these points. 


\subsection{Closed-loop Properties}

The following theorem gives the properties of the Lyapunov-based EMPC algorithm.

Theorem 8.1. Consider the Lyapunov-based EMPC scheme (8.3) with $W, h$ and $O$ from Definition 8.1. Then for all $x(0) \in O$ with $\rho \geq \tilde{\rho}$ such that $\Omega_{\rho} \subseteq \mathbb{X}$ and $x(0) \in \Omega_{\rho}$ the following statements hold:

(i) The scheme is recursively feasible and $x(t) \in \Omega_{\rho}$ for all $t \geq 0$.

(ii) There is $\tilde{t}>0$ with $x(t) \in \Omega_{\tilde{\rho}}$ for all $t \geq \tilde{t}$.

(iii) If $t^{\prime}<\infty$ then $x(t) \rightarrow x_{s}$ as $t \rightarrow \infty$.

Proof. Since the discrete time setting considered here differs from the continuous time setting in the literature and since the proof is rather short, we give a complete proof of this theorem.

We first note that in every step either constraint (8.3e) or constraint (8.3f) is enforced. Together with the fact that $x(t+1)=x(1 \mid t)$ this implies

$$
\begin{aligned}
W(x(t+1)) & =W(x(1 \mid t)) \\
& \leq \max \{W(f(x(t), h(x(t)))), \tilde{\rho}\} \\
& \leq \max \left\{W(x(t))-\alpha_{3}\left(\left|x(t)-x_{s}\right|\right), \tilde{\rho}\right\}
\end{aligned}
$$

where the inequality holds for the first term in the max whenever constraint 8.3f was enforced. Also, recall that $x \in \Omega_{\rho}$ if and only if $W(x) \leq \rho$.

(i): Observe that recursive feasibility follows from $x(t) \in \Omega_{\rho}$ since $\Omega_{\rho} \subseteq \mathbb{X}$. This property now follows by an easy induction. For $t=0$ it follows from the assumption and for $t \rightarrow t+1$ it follows since $x(t) \in \Omega_{\rho}$ implies $\max \left\{W(x(t))-\alpha_{3}\left(\left|x(t)-x_{s}\right|\right), \tilde{\rho}\right\} \leq$ $\max \{\rho, \tilde{\rho}\} \leq \rho$, hence 8.4 yields the assertion.

(ii): For $x \in O \backslash \Omega_{\tilde{\rho}}$ we have $W(x) \geq \tilde{\rho}$ which by (8.1) implies $\left|x-x_{s}\right| \geq \alpha_{2}^{-1}(\tilde{\rho})$ and thus $\alpha_{3}\left(\left|x-x_{s}\right|\right) \geq \alpha_{3}\left(\alpha_{2}^{-1}(\tilde{\rho})\right)=: \delta>0$ for all $x \in O \backslash \Omega_{\tilde{\rho}}$. An induction based on (8.4) similar as in (i) then implies

$$
W(x(t)) \leq \max \{W(x(t))-t \delta, \tilde{\rho}\}
$$

From this, (ii) immediately follows. 
(iii): For $t \geq t^{\prime}, 8.4$ changes to

$$
W(x(t+1)) \leq W(x(t))-\alpha_{3}\left(\left|x(t)-x_{s}\right|\right) .
$$

This implies that $t \rightarrow W(x(t)$ is strictly decreasing and since $W(x)$ is bounded from below by 0 it hence converges to a value $\rho_{\infty}$. We claim that $\rho_{\infty}=0$. Indeed, if $\rho_{\infty}>0$, then $x(t) \in \Omega_{\rho_{\infty}}$ for all $t \in \mathbb{N}$. As in (ii) we obtain $\alpha_{3}\left(\left|x-x_{s}\right|\right) \geq \alpha_{3}\left(\alpha_{2}^{-1}\left(\rho_{\infty}\right)\right)=: \delta_{\infty}>0$ for all $x \in O \backslash \Omega_{\rho_{\infty}}$. This implies $W(x(t)) \leq W\left(x\left(t^{\prime}\right)\right)-\left(t-t^{\prime}\right) \delta_{\infty}$ for all $t \geq t^{\prime}$, which contradicts $W(x(t)) \geq \rho_{\infty}$. Hence $\rho_{\infty}=0$ and thus $W(x(t)) \rightarrow 0$ as $t \rightarrow \infty$ which by (8.2) implies $\left|x(t)-x_{s}\right| \rightarrow 0$, i.e., $x(t) \rightarrow x_{s}$.

Remark 8.1. Using the proof technique from (Grüne and Pannek, 2017, Thm. 2.19) one may also establish asymptotic stability of the set $\Omega_{\tilde{\rho}}$ in the first mode and asymptotic stability of $x_{s}$ in the second mode.

Remark 8.2 (Continuous-time setting). In continuous time, the condition (8.2) changes to

$$
\frac{d W}{d x}(x) f(x, h(x)) \leq-\alpha_{3}\left(\left|x-x_{s}\right|\right)
$$

Consequently, the constraint 8.3f becomes

$$
\frac{d W}{d x}(x(\tau \mid t)) f(x(\tau \mid t), u(\tau \mid t)) \leq \frac{d W}{d x}(x(\tau \mid t)) f(x(\tau \mid t), h(x(\tau \mid t))) .
$$

This constraint should be checked for all $\tau$ from the sampling interval $[t, t+\Delta]$ in order to ensure decrease of the Lyapunov function. However, this is numerically infeasible, which is why it is usually only checked for $t=t_{k}$. The resulting error may prevent the solutions to converge exactly to $\Omega_{\tilde{\rho}}$ and $x_{s}$, which is why additional error terms show up in the continuous time versions of Theorem 8.1, see (Heidarinejad et al., 2012, Thm. 1) or (Ellis, Liu, et al., 2017, Thm. 4.1).

Various modifications and extensions of the basic algorithm described in this section have been presented in the literature. For details we refer to (Ellis, Durand, et al., 2014) or to (Ellis, Liu, et al., 2017) and the references therein.

It should be noted that the additional constraints involving $W$ can significantly affect the economic performance, as the constraints change the behavior of the closed-loop trajectory. Another limitation of the method is that the Lyapunov function $W$ and the corresponding control law $h$ must be known in order to implement the method. While a certain performance loss is, in general, unavoidable, if one wants to enforce stabilitylike behavior that the economically optimal trajectories do not exhibit, the next section 
describes a conceptually similar method in which the explicit knowledge of $W$ and $h$ is not needed. 



\section{Multi-objective Approach}

It is well known that - contrary to EMPC - in stabilizing MPC the optimal value function can be used as a Lyapunov function. In other words, a Lyapunov function can be generated by solving an optimal control problem. Essentially, this Lyapunov function will replace the a priori Lyapunov function $W$ used in the Lyapunov based EMPC approach. This leads to an optimal control problem involving two costs - the economic costs $\ell$ and the stabilizing cost $\ell^{s t a b}$ - and thus the approach is termed a multi-objective approach in (Zavala, 2015), which forms the basis for the subsequent considerations.

\subsection{Derivation of the Scheme}

To this end, we note that for stabilizing MPC either suitable assumptions or suitable terminal conditions must be satisfied in order to guarantee that the optimal value function is indeed a Lyapunov function, similar to what we discussed in the previous sections for EMPC. As in Corollaries 2.1 and 3.1, here we use the simplest possible terminal condition, i.e., we require $x(N \mid t)=x_{s}$ for the equilibrium $x_{s}$. With $\mathbb{X}_{N}$ we denote the set of initial conditions for which this constraint is feasible for given horizon $N$.

The resulting stabilizing optimal control problem with $x=x(t)$ then reads

$$
\min _{u(\cdot \mid t)} J^{s t a b}(x(t), u(\cdot \mid t))=\sum_{k=0}^{N-1} \ell^{s t a b}(x(k \mid t), u(k \mid t))
$$

subject to

$$
\begin{aligned}
x(k+1 \mid t) & =f(x(k \mid t), u(k \mid t)), & & k=0, \ldots, N-1 \\
x(0 \mid t) & =x(t) & & \\
(x(k \mid t), u(k \mid t))^{\top} & \in \mathbb{X} \times \mathbb{U}, & & k=0, \ldots, N-1 \\
x(N \mid t) & =x_{s} . & &
\end{aligned}
$$


We define the optimal value function of this optimal control problem as

$$
V^{s t a b}(x):=\min \left\{J^{s t a b}(x, u) \mid u \in \mathbb{U}^{N}, 9.1 \mathrm{~b}-9.1 \mathrm{e} \text { holds }\right\}
$$

We now assume that the stabilizing cost $\ell^{s t a b}$ satisfies the (in)equalities

$$
\ell^{s t a b}\left(x_{s}, u_{s}\right)=0 \quad \text { and } \quad \ell^{s t a b}(x, u) \geq \alpha_{4}\left(\left|x-x_{s}\right|\right)
$$

where $u_{s}$ is an equilibrium control value, i.e., $f\left(x_{s}, u_{s}\right)=x_{s}$, and $\alpha_{4} \in \mathcal{K}_{\infty}$. We moreover assume that there is $\alpha_{5} \in \mathcal{K}_{\infty}$ such that

$$
V^{s t a b}(x) \leq \alpha_{5}\left(\left|x-x_{s}\right|\right)
$$

holds. Then $V^{s t a b}$ satisfies the inequalities

$$
\alpha_{4}\left(\left|x-x_{s}\right|\right) \leq V^{s t a b}(x) \leq \alpha_{5}\left(\left|x-x_{s}\right|\right)
$$

and

$$
V^{s t a b}(x(t+1)) \leq V^{s t a b}(x(t))-\alpha_{4}\left(\left|x(t)-x_{s}\right|\right)
$$

and is thus a Lyapunov function for the MPC closed loop in the sense of Definition 8.1, from which asymptotic stability of $x_{s}$ follows. The proof of inequality (9.5) was given in Step 2 of the proof of Theorem 2.1.

Now the interesting observation is that this proof still works if the optimal pair $\left(x^{\star}(\cdot \mid t), u^{\star}(\cdot \mid t)\right)$ for the stabilizing problem is replaced by any other admissible trajectory/control pair $(\hat{x}(\cdot), \hat{u}(\cdot))$ satisfying the constraints 9.1b-9.1e). This yields

$$
V^{s t a b}(f(x(t), \hat{u}(0))) \leq J^{s t a b}(x(t), \hat{u})-\alpha_{4}\left(\left|x(t)-x_{s}\right|\right)
$$

instead of 9.5 .

Now the idea is to ensure that the controls $u^{\star}(\cdot \mid t)$ minimizing the economic functional satisfy the constraint

$$
J^{s t a b}\left(x(t+1), u^{\star}(\cdot \mid t+1)\right) \leq(1-\sigma) V^{s t a b}(x(t+1))+\sigma J^{s t a b}\left(x(t), u^{\star}(\cdot \mid t)\right.
$$

for a fixed parameter $\sigma \in[0,1)$ when optimizing the economic criterion, where $u^{\star}(\cdot \mid t)$ denotes the economically optimal control at time $t$. From 9.6$)$ with $\hat{u}=u^{\star}(\cdot \mid t-1)$, for each $\sigma \in[0,1)$ it follows that $(1-\sigma) V^{s t a b}(x(t+1))+\sigma J^{s t a b}\left(x(t), u^{\star}(\cdot \mid t) \geq V^{s t a b}(x(t+1))\right.$, 
hence this constraint is feasible. When it is satisfied, using again (9.6) yields

$$
\begin{aligned}
& J^{\text {stab }}\left(x(t+1), u^{\star}(\cdot \mid t+1)\right) \\
& \leq(1-\sigma) V^{\text {stab }}(x(t+1))+\sigma J^{s t a b}\left(x(t), u^{\star}(\cdot \mid t)\right. \\
& \leq(1-\sigma)\left(J^{s t a b}\left(x(t), u^{\star}(\cdot \mid t)-\alpha_{4}\left(\left|x(t)-x_{s}\right|\right)\right)+\sigma J^{s t a b}\left(x(t), u^{\star}(\cdot \mid t)\right.\right. \\
& \leq J^{\text {stab }}\left(x(t), u^{\star}(\cdot \mid t)-(1-\sigma) \alpha_{4}\left(\left|x(t)-x_{s}\right|\right) .\right.
\end{aligned}
$$

From this it is easy to conclude that $J^{\text {stab }}\left(x(t), u^{\star}(\cdot \mid t)\right)$ converges to 0 and thus $x(t) \rightarrow x_{s}$ follows from the lower bound in (9.4).

Abbreviating $\gamma(t):=(1-\sigma) V^{s t a b}(x(t))+\sigma J^{s t a b}\left(x(t-1), u^{\star}(\cdot \mid t-1)\right.$ for $t \geq 1$ and setting $\gamma(0):=\infty$, the economic optimization problem to be solved in each step of the MPC loop thus reads as follows.

$$
\min _{u(\cdot \mid t)} J\left(x(t), u(\cdot \mid t)=\sum_{k=0}^{N-1} \ell(x(k \mid t), u(k \mid t))\right.
$$

subject to

$$
\begin{array}{rlrl}
x(k+1 \mid t) & =f(x(k \mid t), u(k \mid t)), & & k=0, \ldots, N-1 \\
x(0 \mid t) & =x(t) & & \\
(x(k \mid t), u(k \mid t))^{\top} & \in \mathbb{X} \times \mathbb{U}, \quad \\
x(N \mid t) & =x_{s} \\
J^{s t a b} & (x(t), u(\cdot \mid t+1)) \leq \gamma(t) . &
\end{array}
$$

We note that in every step of this scheme two optimal control problems have to be solved, one in order to determine $u^{\star}(\cdot \mid t)$ and one in order to compute $V^{\text {stab }}(x(t))$ which is needed for evaluating $\gamma(t)$.

\subsection{Closed-loop Properties}

We summarize the properties of this MPC scheme in the following theorem.

Theorem 9.1. Consider the EMPC scheme (9.8) and assume that the underlying stabilizing optimal control problem satisfies 9.2 and (9.4). Then for all $x(0) \in \mathbb{X}$ the MPC closed loop solution $x(t)$ converges to $x_{s}$ as $t \rightarrow \infty$.

Proof. With the same argument as used for $W$ in the proof of Theorem 8.1, we can 
conclude from (9.7) that $J^{s t a b}\left(x(t), u^{\star}(\cdot \mid t)\right) \rightarrow 0$ as $t \rightarrow \infty$. Now the obvious lower bound $\alpha_{4}\left(\left|x(t)-x_{s}\right|\right) \leq J^{s t a b}\left(x(t), u^{\star}(\cdot \mid t)\right) \rightarrow 0$ implies $\alpha_{4}\left(\left|x(t)-x_{s}\right|\right) \rightarrow 0$ and thus $\left|x(t)-x_{s}\right| \rightarrow 0$ as $t \rightarrow \infty$, which shows the claim.

Remark 9.1. (i) Contrary to what is claimed in (Zavala, 2015), it is not clear to us whether in addition to the convergence $x(t) \rightarrow x_{s}$ asymptotic stability does also hold. The reason for this is that in the first step of the algorithm due to $\gamma(0)=\infty$ no stabilizing constraint is imposed. Indeed, this constraint cannot be implemented in the first time step $t=0$ because no value $J^{\text {stab }}\left(x(t-1), u^{\star}(\cdot \mid t-1)\right)$ from the previous time step is available. This, however, means that the economic optimization criterion may yield an optimal control which steers the system away from $x_{s}$ for initial conditions $x(0) \approx x_{s}$, or even $x(0)=x_{s}$, contradicting stability of $x_{s}$ for the closed loop.

(ii) The parameter $\sigma$ defines the desired "degree of decrease" of $J^{\text {stab }}$ and thus the speed of convergence of $x(t)$ to $x_{s}$. There is thus a tradeoff between the two objectives $J$ and $J^{\text {stab }}$. Under suitable convexity conditions it can be shown that the optimal solution computed in each step is weakly Pareto optimal for the multi-objective optimization problem defined by these two criteria, see (Zavala, 2015). In this context, $\sigma$ determines the location of the Pareto optimum on the Pareto front. However, these considerations only apply to the open loop optimal solutions in each step of the MPC scheme and do not allow for an easy estimate on the performance of the closed loop.

(iii) We note that while the constraint (9.8f) is feasible, for non-convex problems it is not guaranteed that the optimization algorithm will find the feasible solution. One way to cope with this problem is to choose suitable intializations for the optimization algorithm, e.g., the optimal control from the computation of $V^{s t a b}(x(t))$, whose computation in turn should be initialized with the control sequence $\tilde{u}$ used in the derivation of $(9.6)$ in order to guarantee the necessary decay. Further strategies to deal with this problem are discussed in (Zavala, 2015).

\subsection{Example - Chemical Reactor without Dissipativity}

We reconsider the chemical reactor model without dissipativity from Section 3.4. We use the same economic stage cost $\ell$ as before and the stabilizing stage cost $\ell(x, u)=$ $\left\|x-x_{s}\right\|^{2}+\left(u-u_{s}\right)^{2}$, with $x_{s}$ and $u_{s}$ from the steady state analysis in Section 3.4 .

Figures $9.1-(9.3)$ show the resulting closed loop trajectories. It is clearly visible that the solution converges to the optimal steady state the faster, the smaller $\sigma$ is. We also note the obvious similarities between Figure 9.1 and Figure 3.5, where in both cases a 
fast convergence to $\left(x_{s}, u_{s}\right)$ is enforced, and between Figure 9.3 and Figure 3.3 , where only a very slow or no convergence, at all, to $\left(x_{s}, u_{s}\right)$ is enforced.
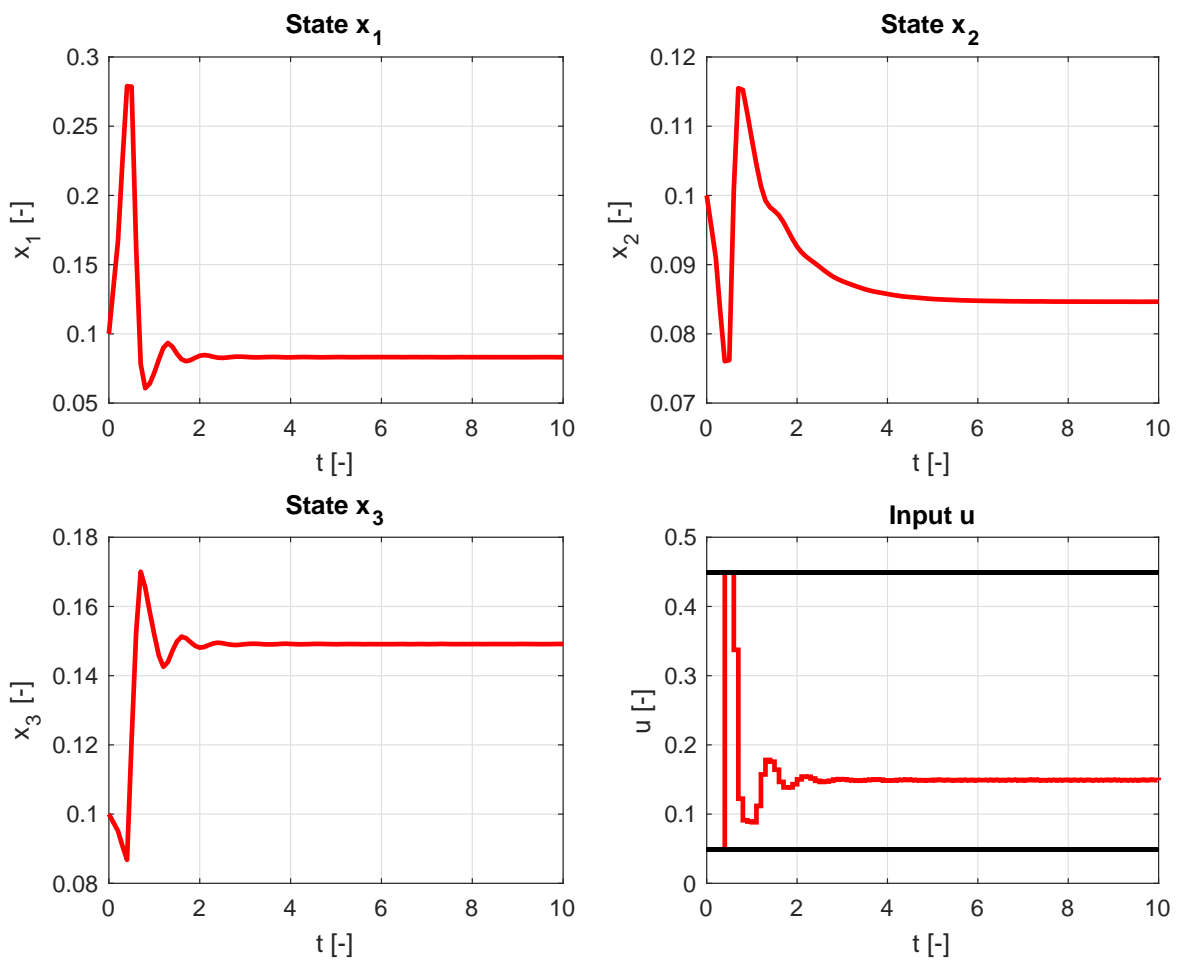

Figure 9.1: Closed-loop multi-objective EMPC for system 3.10 with $\sigma=0.5$. 

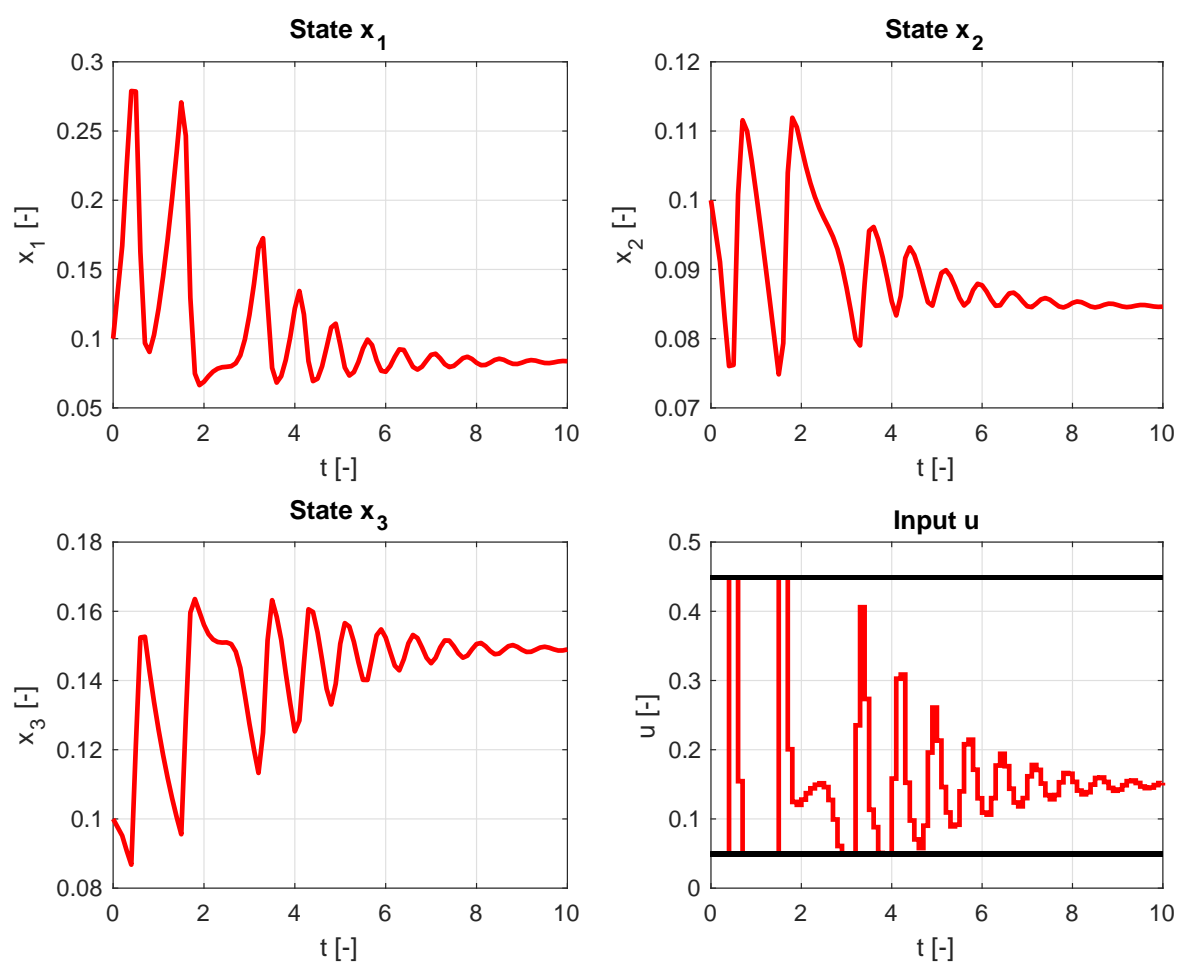

Figure 9.2: Closed-loop multi-objective EMPC for system 3.10 with $\sigma=0.9$.
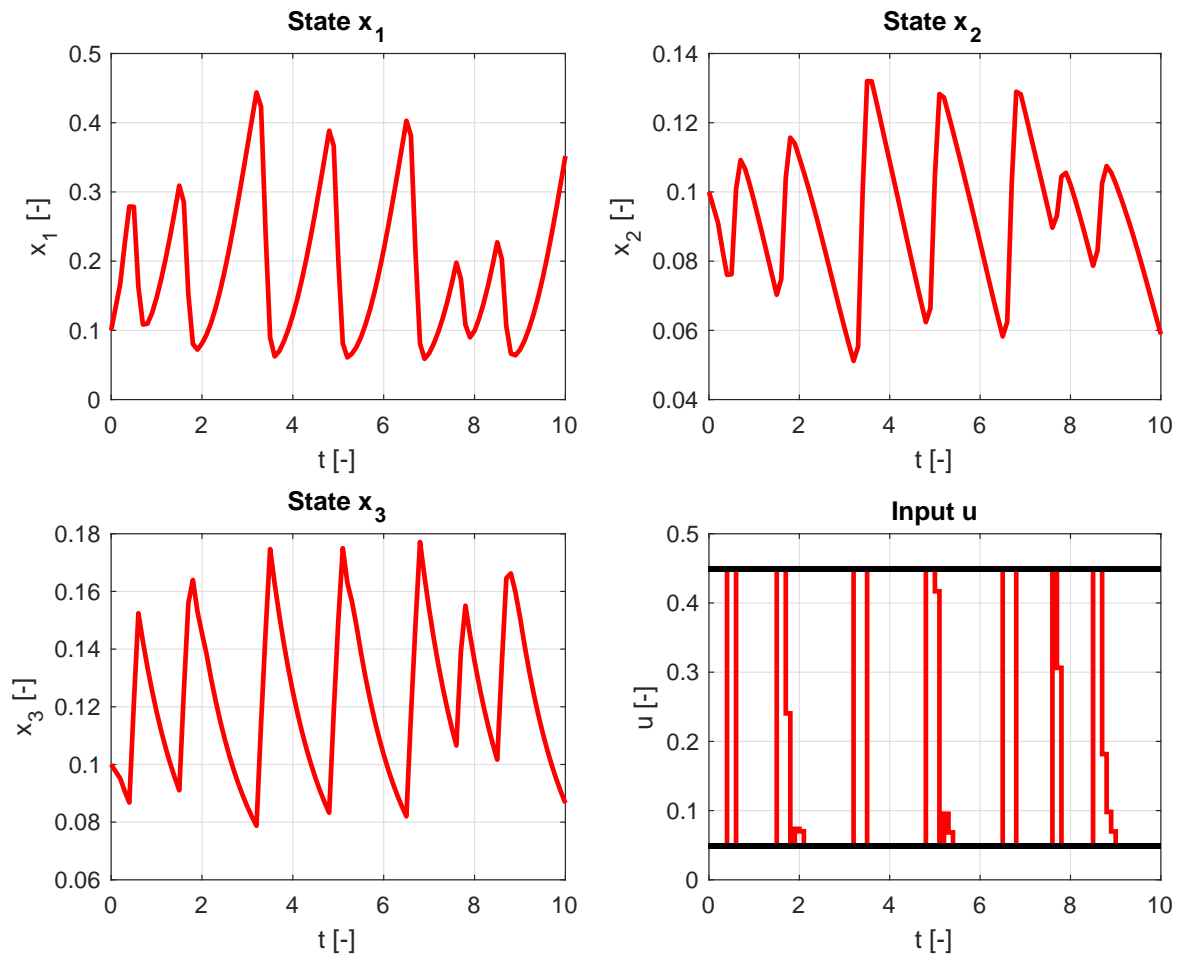

Figure 9.3: Closed-loop multi-objective EMPC for system 3.10 with $\sigma=0.99$. 


\section{Conclusions and Outlook}

\subsection{Discussion}

In this survey, we have reviewed various economic MPC schemes that have been developed in recent years. The schemes differ in how the underlying OCP is formulated (with/without terminal or other stability-related constraints), what a priori knowlege is required for implementing the scheme (the optimal steady-state, a suitable Lyapunov function, etc.), and which properties can be established for the resulting closed-loop system (averaged/non-averaged performance statements, stability/convergence, (average) constraint satisfaction, etc.). A concise comparison of the presented schemes with respect to these issues is given in Table 10.1 below. 


\begin{tabular}{|c|c|c|c|c|c|c|}
\hline Scheme & $\begin{array}{l}\text { Core } \\
\text { Assumptions }\end{array}$ & $\begin{array}{l}\text { Required } \\
\text { Pre-Knowledge }\end{array}$ & $\begin{array}{l}\text { Term. Constraints } \\
\text { and Penalties }\end{array}$ & $\begin{array}{l}\text { Closed-loop } \\
\text { Performance }\end{array}$ & $\begin{array}{l}\text { Stability } \\
\text { Properties }\end{array}$ & Remarks \\
\hline $\begin{array}{l}\text { Terminal } \\
\text { Constraints } \\
\text { (Section 33 }\end{array}$ & $\begin{array}{l}\text { strict dissipativtiy and } \\
\text { finite-time reach. of } \mathbb{X}_{f}\end{array}$ & $\begin{array}{l}\text { opt. steady state } x_{s} \\
\text { terminal penalty } \\
\text { and constraint }\end{array}$ & $\begin{array}{l}\text { terminal penalty and } \\
\text { constraint around } x_{s}\end{array}$ & $\begin{array}{l}\text { asymp. avg. perf. } \\
\leq \ell\left(x_{s}, u_{s}\right) \text {, } \\
\text { transient perf. } \\
\text { estimates available }\end{array}$ & $\begin{array}{l}\text { asymptotic } \\
\text { stability }\end{array}$ & \\
\hline $\begin{array}{l}\text { Turnpike } \\
\text { Approach } \\
\text { (Section } 4\end{array}$ & $\begin{array}{l}\text { strict dissipativity and } \\
\text { exp. reachability of } x_{s}\end{array}$ & none & none & $\begin{array}{l}\text { asymp. avg. perf. } \\
\leq \ell\left(x_{s}, u_{s}\right)+\delta(N), \\
\text { transient perf. } \\
\text { estimates available }\end{array}$ & $\begin{array}{l}\text { practical } \\
\text { stability }\end{array}$ & $\begin{array}{l}\text { recursive } \\
\text { feasibility } \\
\text { for long } \\
\text { horizons }\end{array}$ \\
\hline $\begin{array}{c}\text { Averaged } \\
\text { Constraints } \\
\text { (Section } \sqrt{6}\end{array}$ & $\begin{array}{l}\text { strict dissipativity and } \\
\text { finite-time reach. of } \mathbb{X}_{f}\end{array}$ & $\begin{array}{l}\text { terminal penalty } \\
\text { and constraint }\end{array}$ & $\begin{array}{l}\text { terminal penalty and } \\
\text { constraint around } x_{s}\end{array}$ & $\begin{array}{l}\text { asymp. avg. perf. } \\
\leq \ell\left(x_{s}, u_{s}\right)\end{array}$ & $\begin{array}{l}\text { asymptotic } \\
\text { convergence }\end{array}$ & \\
\hline $\begin{array}{l}\text { Generalized } \\
\text { Term. Constr. } \\
\text { (Section 7) }\end{array}$ & $\begin{array}{l}\text { finite-time reachability } \\
\text { of some steady state }\end{array}$ & none & $\begin{array}{l}\text { gen. term. constraint } \\
\text { and term. penalty }\end{array}$ & $\begin{array}{l}\text { asymp. avg. perf. } \\
\leq \kappa_{\infty}\end{array}$ & - & \\
\hline $\begin{array}{l}\text { Lyapunov-based } \\
\text { Approach } \\
\text { (Section } 8\end{array}$ & $\begin{array}{l}\text { existence of stab. } \\
\text { feedback and corr. } \\
\text { Lyapunov function }\end{array}$ & $\begin{array}{l}\text { a priori known } \\
\text { stab. feedback and } \\
\text { Lyapunov function }\end{array}$ & $\begin{array}{l}\text { terminal penalty and } \\
\text { constraint given by Lyap. } \\
\text { function and its domain }\end{array}$ & & $\begin{array}{l}\text { asymptotic } \\
\text { stability }\end{array}$ & \\
\hline $\begin{array}{l}\text { Multi-objective } \\
\text { Approach } \\
\text { (Section } 9\end{array}$ & $\begin{array}{l}\text { stabilization with track. } \\
\text { NMPC required and } \\
\text { finite-time reach. of } \mathbb{X}_{f}\end{array}$ & $\begin{array}{l}\text { desired reference } x_{s} \\
\text { corr. terminal penalty } \\
\text { and constraint }\end{array}$ & $\begin{array}{l}\text { terminal penalty and } \\
\text { constraint around } x_{s}\end{array}$ & $\begin{array}{l}\text { asymp. avg. perf. } \\
\text { equals } \ell\left(x_{s}, u_{s}\right)\end{array}$ & $\begin{array}{l}\text { asymptotic } \\
\text { convergence }\end{array}$ & \\
\hline
\end{tabular}


In summary, for the schemes with (classical) terminal constraints (Section 3) and without such terminal constraints (Section 4), optimal steady-state operation as well as closed-loop (practical) asymptotic stability of the optimal steady-state can be characterized via a suitable (strict) dissipativity condition. Furthermore, for these schemes, closed-loop averaged and non-averaged infinite-horizon performance guarantees as well as transient performance guarantees can be given, again (mostly) based on the same (strict) dissipativity condition (Section 5). Section 6 discussed how average constraints can be incorporated into EMPC schemes. Again under a suitably relaxed (strict) dissipativity condition, optimal steady-state operation and closed-loop convergence to the optimal steady-state follow. Compared to the basic case without average constraints, most of the existing results are only available for schemes including terminal constraints. Economic MPC with generalized terminal constraints (Section 7) can be seen as an "intermediate" approach between using no or fixed terminal constraints. Such generalized terminal constraints allow for closed-loop (average) performance statements without the a priori knowledge of the optimal steady-state and without using a dissipativity condition. On the other hand, convergence to the (globally) optimal operating behavior is not necessarily guaranteed. Lyapunov-based EMPC (Section 8) employs the knowledge of an a priori known Lyapunov function $W$ in order to ensure closed-loop boundedness inside a sublevel set of $W$, and (if desired) also convergence to the optimal steady-state. Finally, if convergence to the optimal steady-state should be ensured and no a priori known Lyapunov function $W$ is available, the multi-objective approach (Section 9) can be used. Here, an additional (auxiliary) OCP with a stabilizing (positive definite) cost function is solved at each time step, whose optimal value function in turn is used as an additional stabilizing constraint in the economic MPC problem.

In conclusion, the closed-loop behavior resulting from application of EMPC schemes is by now fairly well understood - at least in the basic case where steady-state operation is optimal and where there is no plant-model mismatch - both in terms of performance and convergence to the optimal steady-state. This is true for both basic MPC categories with and without additional terminal constraints.

\subsection{Further Results and Open Problems}

Finally, we briefly comment on existing further results (without aiming for a complete picture of all available papers in the field of economic MPC) and hint at some open problems that deserve investigation. Open issues concerning the different MPC meth- 
ods described in this survey were already pointed out in various places in the single sections. This includes considering, e.g., average constraints in economic MPC schemes without terminal constraints, and (transient) performance guarantees for the economic MPC schemes of Sections 6, 9 ,

The closed-loop performance and stability analysis in Sections 35 was shown for the basic case where steady-state operation is optimal. However, as we have seen in Section 3.4, it may happen that a system it not optimally operated at steady state. Most of the results presented in Sections 34 have been extended to more general settings where, e.g., periodic operation is optimal. In this case, optimal periodic operation can again (almost) equivalently be characterized by a suitable (strict) dissipativity condition (Müller, Grüne, and Allgöwer, 2015), which is also sufficient for closed-loop convergence to the optimal periodic orbit in both settings with (Zanon, Grüne, et al., 2017) and without (Müller and Grüne, 2016b) terminal constraints. A continuous-time extension to more general time-varying turnpikes is discussed in (Alessandretti et al., 2016). However, therein no procedure for classification of time-varying turnpikes is given.

Another important direction for future research are EMPC schemes with time-varying problem data. Already the case of time-invariant dynamics and constraints combined with a time-varying cost functional can lead to considerable difficulties. An important special case of this setting are discounted OCPs as they frequently arise in Economics (Carlson et al., 1991). The application of EMPC as a mean of approximating infinitehorizon solutions to discounted OCPs arising in Economics is investigated in (Grüne, Semmler, et al., 2015). While the main performance results discussed in this paper carry over to EMPC for discounted problems if the optimal control problem exhibits the turnnpike property, the difficulty in the discounted case lies in ensuring that the turnpike property holds. Interestingly, these conceptual difficulties already occur for tracking NMPC (Gaitsgory et al., 2015).

Moreover, while for OCPs with time-invariant data the time-invariant turnpike (if it exists) is easily classified as the best reachable steady-state, the classification of timevarying turnpikes appears to be quite difficult, whenever they are not periodic orbits.

In contrast to Section 9, wherein the auxiliary objective is considered as a constraint, so-called dual objective NMPC schemes combine an economic objective in a weighted sum with a tracking objective, cf. (Maree and Imsland, 2016). Although the main idea of combining multiple objectives appears to be not entirely new (Böhm et al., 2008), there is no general framework for dual objective NMPC design. It also has been suggested to design tracking MPC such that locally economic costs are approximated (Zanon, Gros, et al., 2014). 
The results presented in this survey article have been obtained under the strong assumption that we have a perfect model, i.e., no disturbances/uncertainties or model/plant mismatch is present. For most practical applications, studying the influence of disturbances on the closed-loop system is of paramount importance. This is in particular the case in economic MPC, where disturbances should not just be counteracted (as is typically done in stabilizing robust MPC schemes), but potentially (economically) beneficial disturbances should be taken advantage of. To this end, it was noted in (Bayer, Müller, et al., 2014) that just transferring robust MPC approaches from a stabilizing to an economic context might not result in an optimal closed-loop performance, necessitating the development of novel schemes. Some robust and stochastic economic MPC approaches can, e.g., be found in (Bayer, Lorenzen, et al., 2016; Bayer, Müller, et al., 2014; Broomhead et al., 2015; Huang et al., 2012, Lucia et al., 2014 Marquez et al., 2014 Sokoler et al., 2014). Overall, the picture is still much less complete compared to the nominal case, and various interesting open problems remain. This includes robustness of economic MPC schemes without terminal constraints, performance estimates, or a classification of the optimal operating behavior under disturbances.

With respect to the extension of distributed MPC and output-feedback MPC to economic settings, there appears to be a lot of room for further investigations. First distributed economic MPC schemes have, e.g., been proposed in (Braun et al., 2016; Driessen et al., 2012; Köhler et al., 2016; Lee and Angeli, 2011) for certain cooperative and competitive settings.

As claimed by Maciejowski, 2002 MPC has had a significant impact on industrial process control. The same is not yet true for EMPC. To this end, the integration of EMPC schemes into existing architectures for operation of large-scale plants still requires extensive research. 



\section{Bibliography}

Alessandretti, A., A. Aguiar, and C. Jones (2014). "An Economic Model Predictive Control scheme with terminal penalty for continuous-time systems". In: Proc. of the 53rd IEEE Conference on Decision and Control (CDC), pp. 2728-2733.

Alessandretti, A., A.P. Aguiar, and C.N. Jones (2016). "On convergence and performance certification of a continuous-time economic model predictive control scheme with timevarying performance index". In: Automatica 68, pp. 305-313.

Amrit, R., J.B. Rawlings, and D. Angeli (2011). "Economic optimization using model predictive control with a terminal cost". In: Annual Reviews in Control 35.2, pp. 178186. ISSN: $1367-5788$.

Angeli, D., R. Amrit, and J.B. Rawlings (2012). "On Average Performance and Stability of Economic Model Predictive Control". In: IEEE Transactions on Automatic Control 57.7, pp. 1615-1626.

Bailey, J.E., F.J.M. Horn, and R.C. Lin (1971). "Cyclic operation of reaction systems: Effects of heat and mass transfer resistance". In: AIChE Journal 17.4, pp. 818-825.

Bayer, Florian A., Matthias Lorenzen, Matthias A. Müller, and Frank Allgöwer (2016). "Robust economic Model Predictive Control using stochastic information". In: Automatica 74, pp. 151-161.

Bayer, Florian A., Matthias A. Müller, and Frank Allgöwer (2014). "Tube-based robust economic model predictive control". In: Journal of Process Control 24.8, pp. 12371246.

Bertsekas, D. (1999). Nonlinear Programming. 2nd. Athena Scientific, Belmont, Massachusetts.

Böhm, C., R. Findeisen, and F. Allgöwer (2008). "Avoidance of Poorly Observable Trajectories: A predictive control perspective". In: Proc. 17th IFAC World Congress. Vol. 41. 2. Elsevier, pp. 1952-1957.

Braun, P., L. Grüne, C. M. Kellett, S. R. Weller, and K. Worthmann (2016). "A distributed optimization algorithm for the predictive control of smart grids". In: IEEE Transactions on Automatic Control 61.12, pp. 3898-3911. 
Broomhead, Timothy J., Chris Manzie, Rohan C. Shekhar, and Peter Hield (2015). "Robust periodic economic MPC for linear systems". In: Automatica 60, pp. 30-37.

Carlson, D.A., A. Haurie, and A. Leizarowitz (1991). Infinite Horizon Optimal Control: Deterministic and Stochastic Systems. Springer Verlag.

Chen, H. and F. Allgöwer (1998). "A quasi-infinite horizon nonlinear model predictive control scheme with guaranteed stability". In: Automatica 34.10, pp. 1205-1217.

Damm, T., L. Grüne, M. Stieler, and K. Worthmann (2014). "An exponential turnpike theorem for dissipative optimal control problems". In: SIAM Journal on Control and Optimization 52.3, pp. 1935-1957.

Diehl, M., R. Amrit, and J.B. Rawlings (2011). "A Lyapunov function for economic optimizing model predictive control". In: IEEE Transactions on Automatic Control 56.3, pp. 703-707.

Driessen, P. A. A., R. M. Hermans, and P. P. J. van den Bosch (2012). "Distributed economic model predictive control of networks in competitive environments". In: Proceedings of the 51st IEEE Conference on Decision and Control, pp. 266-271.

Ebenbauer, C., T. Raff, and F. Allgöwer (2009). "Dissipation inequalities in systems theory: An introduction and recent results". In: R. Jeltsch and G. Wanner (ed.), Invited Lectures of the International Congress on Industrial and Applied Mathematics 200\%, pp. 23-42.

Ellis, M., H. Durand, and P.D. Christofides (2014). "A tutorial review of economic model predictive control methods". In: Journal of Process Control 24.8, pp. 1156-1178.

Ellis, M., M. Liu, and P. Christofides (2017). Economic Model Predictive Control: Theory, Formulations and Chemical Process Applications. Springer, Berlin.

Engell, S. (2007). "Feedback control for optimal process operation". In: Journal of Process Control 17, pp. 203-219.

Fagiano, L. and A. R. Teel (2013). "Generalized terminal state constraint for model predictive control". In: Automatica 49.9, pp. 2622-2631.

Faulwasser, T. and D. Bonvin (2015a). "On the Design of Economic NMPC Based on an Exact Turnpike Property". In: Proceedings of the 9th IFAC Symposium on Advanced Control of Chemical Processes - ADCHEM 2015. Vol. 48. 8. IFAC-PapersOnLine, pp. 525-530.

- (2015b). "On the Design of Economic NMPC based on Approximate Turnpike Properties". In: 54th IEEE Conference on Decision and Control. Osaka, Japan, pp. 4964 4970 .

- (2017). "Exact Turnpike Properties and Economic NMPC". In: European Journal of Control 35, pp. 34-41. 
Faulwasser, T., V. Hagenmeyer, and R. Findeisen (2014). "Constrained Reachability and Trajectory Generation for Flat Systems". In: Automatica 50.4, pp. 1151-1159.

Faulwasser, T., M. Korda, C.N. Jones, and D. Bonvin (2014). "Turnpike and Dissipativity Properties in Dynamic Real-Time Optimization and Economic MPC". In: Proc. of the 53rd IEEE Conference on Decision and Control. Los Angeles, California, USA, pp. 2734-2739.

- (2017). "On Turnpike and Dissipativity Properties of Continuous-Time Optimal Control Problems". In: Automatica 81, pp. 297-304.

Ferramosca, A., D. Limon, I. Alvarado, T. Alamo, and E. F. Camacho (2009). "MPC for tracking with optimal closed-loop performance". In: Automatica 45.8, pp. 1975-1978.

Ferramosca, A., D. Limon, and E. F. Camacho (2014). "Economic MPC for a Changing Economic Criterion for Linear Systems". In: IEEE Transactions on Automatic Control 59.10, pp. 2657-2667.

Gaitsgory, Vladimir, Lars Grüne, and Neil Thatcher (2015). "Stabilization with discounted optimal control". In: Systems $\&$ Control Letters 82, pp. 91-98.

Grimm, G., M. J. Messina, S. E. Tuna, and A. R. Teel (2005). "Model predictive control: for want of a local control Lyapunov function, all is not lost". In: IEEE Transactions on Automatic Control 50.5, pp. 546-558.

Grüne, L. (2009). "Analysis and design of unconstrained nonlinear MPC schemes for finite and infinite dimensional systems". In: SIAM Journal on Control and Optimization 48.2, pp. $1206-1228$.

- (2013). "Economic receding horizon control without terminal constraints". In: Automatica 49.3, pp. 725-734.

- (2016). "Approximation properties of receding horizon optimal control". In: Jahresbericht DMV 118.1, pp. 3-37.

Grüne, L. and M.A. Müller (2016). "On the relation between strict dissipativity and turnpike properties". In: Systems \& Control Letters 90, pp. 45-53.

Grüne, L. and A. Panin (2015). "On non-averaged performance of economic MPC with terminal conditions". In: Proc. of the 54 th IEEE Conference on Decision and Control, pp. 2740-2745.

Grüne, L. and J. Pannek (2017). Nonlinear Model Predictive Control. Theory and Algorithms. 2nd ed. London: Springer-Verlag.

Grüne, L., W. Semmler, and M. Stieler (2015). "Using nonlinear model predictive control for dynamic decision problems in economics". In: Journal of Economic Dynamics and Control 60, pp. 112-133. 
Grüne, L. and M. Stieler (2014a). "A Lyapunov function for economic MPC without terminal conditions". In: Proc. of the 53rd IEEE Conference on Decision and Control, pp. 2740-2745.

- (2014b). "Asymptotic stability and transient optimality of economic MPC without terminal conditions". In: Journal of Process Control 24.8, pp. 1187-1196.

Heidarinejad, M., J. Liu, and P. D. Christofides (2012). "Economic model predictive control of nonlinear process systems using Lyapunov techniques". In: AIChE Journal 58.3 , pp. $855-870$.

Hill, David J. and Peter J. Moylan (1980). "Dissipative Dynamical Systems: Basic InputOutput and State Properties". In: Journal of the Franklin Institute 309.5, pp. 327357.

Houska, B., H.J. Ferreau, and M. Diehl (2011). "ACADO toolkit - An open-source framework for automatic control and dynamic optimization". In: Optimal Control Applications and Methods 32.3, pp. 298-312.

Houska, B. and M. A. Müller (2017). "Cost-to-travel functions: a new perspective on optimal and model predictive control". In: System \& Control Letters. submitted.

Huang, R., L. T. Biegler, and E. Harinath (2012). "Robust stability of economically oriented infinite horizon NMPC that include cyclic processes". In: Journal of Process Control 22.1, pp. 51-59.

Jadbabaie, A. and J. Hauser (2005). "On the stability of receding horizon control with a general terminal cost". In: IEEE Transactions on Automatic Control 50.5, pp. 674678.

Jadbabaie, A., J. Yu, and J. Hauser (2001). "Unconstrained receding-horizon control of nonlinear systems". In: IEEE Transactions on Automatic Control 46.5, pp. 776-783.

Kadam, J.V. and W. Marquardt (2007). "Integration of Economical Optimization and Control for Intentionally Transient Process Operation". English. In: Assessment and Future Directions of Nonlinear Model Predictive Control. Ed. by R. Findeisen, F. Allgöwer, and L.T. Biegler. Vol. 358. Lecture Notes in Control and Information Sciences. Springer Berlin Heidelberg, pp. 419-434.

Keerthi, S. S. and E. G. Gilbert (1988). "Optimal infinite horizon feedback laws for a general class of constrained discrete-time systems: stability and moving horizon approximations". In: Journal of Optimization Theory and Applications 57, pp. 265293.

Kellett, C.M. (2014). "A compendium of comparison function results". In: Mathematics of Control, Signals, and Systems 26.3, pp. 339-374. 
Klatt, K.U., S. Engell, A. Kremling, and F. Allgöwer (1995). "Testbeispiel: Rührkesselreaktor mit Parallel- und Folgereaktion". In: Entwurf nichtlinearer Regelungen. Ed. by S. Engell. Oldenbourg-Verlag, pp. 425-432.

Köhler, P. N., M. A. Müller, and F. Allgöwer (2016). "A distributed economic MPC scheme for coordination of self-interested systems". In: Proceedings of the American Control Conference, pp. 889-894.

Lee, C. K. and J. E. Bailey (1980). "Modification of Consecutive-Competitive Reaction Selectivity by Periodic Opera". In: Ind. Eng. Chem. Proc. Des. Dev. 19, pp. 160-166.

Lee, E.B. and L. Markus (1967). Foundations of Optimal Control Theory. The SIAM series in applied mathematics. John Wiley \& Sons New York, London, Sydney.

Lee, J. and D. Angeli (2011). "Cooperative distributed model predictive control for linear plants subject to convex economic objectives". In: Proceedings of the 50th IEEE Conference on Decision and Control and European Control Conference, pp. 3434-3439.

Limon, D., I. Alvarado, T. Alamo, and E. F. Camacho (2008). "MPC for tracking piecewise constant references for constrained linear systems". In: Automatica 44.9, pp. 2382-2387.

Limon, D., M. Pereira, D. Muñoz de la Peña, T. Alamo, and J. M. Grosso (2014). "Single-layer economic model predictive control for periodic operation". In: Journal of Process Control 24.8, pp. 1207-1224.

Lucia, Sergio, Joel A. E. Andersson, Heiko Brandt, Moritz Diehl, and Sebastian Engell (2014). "Handling uncertainty in economic nonlinear model predictive control: A comparative case study". In: Journal of Process Control 24.8, pp. 1247-1259. ISSN: 0959-1524.

Maciejowski, J.M. (2002). Predictive control: with constraints. Pearson Education Limited.

Maree, J.P. and L. Imsland (2016). "Combined economic and regulatory predictive control". In: Automatica 69, pp. 342-347.

Marquez, A., J. Patiño, and J. Espinosa (2014). "Min-max Economic Model Predictive Control". In: Proceedings of the 53rd IEEE Conference on Decision and Control, pp. 4410-4415.

Mayne, D.Q., J.B. Rawlings, C.V. Rao, and P.O.M. Scokaert (2000). "Constrained model predictive control: Stability and optimality". In: Automatica 36.6, pp. 789-814.

Michalska, H. and R.B. Vinter (1994). "Nonlinear stabilization using discontinuous moving-horizon control". In: IMA Journal of Mathematical Control and Information 11.4, pp. 321-340. 
Morari, M., Y. Arkun, and G. Stephanopoulos (1980). "Studies in the synthesis of control structures for chemical processes: Part I: Formulation of the problem. Process decomposition and the classification of the control tasks. Analysis of the optimizing control structures". In: 26.2, pp. 220-232.

Moylan, P. (2014). Dissipative Systems and Stability. http://www.pmoylan.org.

Müller, M. A., D. Angeli, and F. Allgöwer (2014a). "On the performance of economic model predictive control with self-tuning terminal cost". In: Journal of Process Control 24.8, pp. 1179-1186.

- (2014b). "Transient average constraints in economic model predictive control". In: Automatica 50.11, pp. 2943-2950.

Müller, M. A., D. Angeli, F. Allgöwer, R. Amrit, and J. B. Rawlings (2014). "Convergence in economic model predictive control with average constraints". In: Automatica 50.12, pp. $3100-3111$.

Müller, M. A. and L. Grüne (2016a). "Economic model predictive control without terminal constraints for optimal periodic behavior". In: Automatica 70, pp. 128-139.

- (2016b). "Economic model predictive control without terminal constraints for optimal periodic behavior". In: Automatica 70, pp. 128-139.

Müller, M. A., L. Grüne, and F. Allgöwer (2015). "On the role of dissipativity in economic model predictive control". In: Proceedings of the 5th IFAC Conference on Nonlinear Model Predictive Control, pp. 110-116.

Müller, M.A., D. Angeli, and F. Allgöwer (2013). "Economic model predictive control with self-tuning terminal cost". In: European Journal of Control.

- (2015). "On Necessity and Robustness of Dissipativity in Economic Model Predictive Control". In: IEEE Transactions on Automatic Control 60.6, pp. 1671-1676. ISSN: 0018-9286.

Propoi, A.I. (1963). "Application of linear programming methods for the synthesis of automatic sampled-data systems". In: Avtomat. i Telemekh 24.7, pp. 912-920.

Rawlings, J. and R. Amrit (2009). "Optimizing process economic performance using model predictive control". In: Nonlinear Model Predictive Control - Towards New Challenging Applications. Ed. by L. Magni, D. Raimondo, and F. Allgöwer. Vol. 384. Lecture Notes in Control and Information Sciences. Springer Berlin, pp. 119-138.

Rawlings, J.B. and D.Q. Mayne (2009). Model Predictive Control: Theory 8 Design. Nob Hill Publishing, Madison, WI.

Renken, A. (1972). "The use of periodic operation to improve the performance of continuous stirred tank reactors". In: Chemical Engineering Science 27, pp. 1925-1932. 
Rothfuß, R., J. Rudolph, and M. Zeitz (1996). "Flatness based control of a nonlinear chemical reactor model". In: Automatica 32, pp. 1433-1439.

Sokoler, L. E., B. Dammann, H. Madsen, and J. B. Jørgensen (2014). "A mean-variance criterion for economic model predictive control of stochastic linear systems". In: Proceedings of the 53rd Conference on Decision and Control, pp. 5907-5914.

Weiss, Leonard (1972). "Controllability, realization and stability of discrete-time systems". In: SIAM Journal on Control 10.2, pp. 230-251.

Willems, Jan C (2007). "Dissipative dynamical systems". In: European Journal of Control 13.2-3, pp. 134-151.

Willems, J.C. (1972). "Dissipative dynamical systems part I: General theory". In: Archive for rational mechanics and analysis 45.5, pp. 321-351.

Zanon, M., L. Grüne, and M. Diehl (2017). "Periodic optimal control, dissipativity and MPC". In: IEEE Transactions on Automatic Control. to appear.

Zanon, Mario, Sébastien Gros, and Moritz Diehl (2014). "Indefinite linear MPC and approximated economic MPC for nonlinear systems". In: Journal of Process Control 24.8, pp. $1273-1281$.

Zavala, V. M. (2015). "A multiobjective optimization perspective on the stability of economic MPC". In: Proceedings of the 9th IFAC Symposium on Advanced Control of Chemical Processes - ADCHEM 2015. Vol. 48. IFAC Papers OnLine, pp. 974-980. 